\title{
Glucose signal transduction and the role of the HD2 family of histone deacetylases in Arabidopsis seedling germination and development
}

\author{
By: Reem Alhattab
}

\begin{abstract}
A thesis Submitted to the Faculty of Graduate Studies and Research of Carleton University, in partial fulfillment of the requirements for the degree of a Master of Science in Biology.
\end{abstract}

Department of Biology

Carleton University

Ottawa, ON, Canada

2009

(C) Copyright

2009, Reem Alhattab 
Library and Archives

Canada

Published Heritage

Branch

395 Wellington Street

Ottawa ON K1A ON4

Canada
Bibliothèque et

Archives Canada

Direction du

Patrimoine de l'édition

395 , rue Wellington

Ottawa ON K1A ON4

Canada
Your file Votre référence
ISBN: $978-0-494-64440-9$
Our file Notre référence
ISBN: $978-0-494-64440-9$
NOTICE:

The author has granted a nonexclusive license allowing Library and Archives Canada to reproduce, publish, archive, preserve, conserve, communicate to the public by telecommunication or on the Internet, loan, distribute and sell theses worldwide, for commercial or noncommercial purposes, in microform, paper, electronic and/or any other formats.

The author retains copyright ownership and moral rights in this thesis. Neither the thesis nor substantial extracts from it may be printed or otherwise reproduced without the author's permission.
AVIS:

L'auteur a accordé une licence non exclusive permettant à la Bibliothèque et Archives Canada de reproduire, publier, archiver, sauvegarder, conserver, transmettre au public par télécommunication ou par l'Internet, prêter, distribuer et vendre des thèses partout dans le monde, à des fins commerciales ou autres, sur support microforme, papier, électronique et/ou autres formats.

L'auteur conserve la propriété du droit d'auteur et des droits moraux qui protège cette thèse. $\mathrm{Ni}$ la thèse ni des extraits substantiels de celle-ci ne doivent être imprimés ou autrement reproduits sans son autorisation.
In compliance with the Canadian Privacy Act some supporting forms may have been removed from this thesis.

While these forms may be included in the document page count, their removal does not represent any loss of content from the thesis.
Conformément à la loi canadienne sur la protection de la vie privée, quelques formulaires secondaires ont été enlevés de cette thèse.

Bien que ces formulaires aient inclus dans la pagination, il n'y aura aucun contenu manquant.

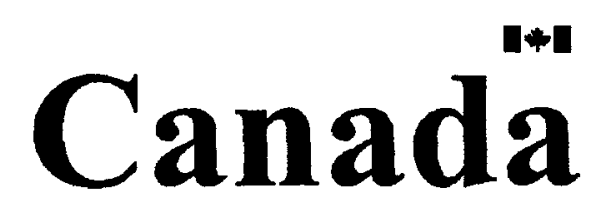




\section{ACKNOWLEDGEMENTS}

I would like to thank my supervisors Dr. Brian Miki and Dr. Tim Xing and Carleton University for giving me the opportunity and support to achieve my goals and complete my graduate studies. I would also like to thank all of the lab members who offered guidance on this project: Hélène Labbé, Teresa Martin, Ming $\mathrm{Hu}$, Nickola Kovinich and Dr. Ashraf Abdeen. I would also like to thank the members of my committee Dr. Doug Johnson, and Dr. Shelley Hepworth for their helpful comments and suggestions on this project. Great thanks to Dr. Owen Rowland for his useful suggestions on this project. Final thanks must be given to my family, and friends for their support. 
TABLE OF CONTENTS

PAGE

ACCEPTANCE FORM

i

ACKNOWLEDGMENTS ii

TABLE OF CONTENTS iii

LIST OF TABLES vi

LIST OF FIGURES vii

LIST OF ABBREVIATIONS ix

ABSTRACT

THESIS SUMMARY Xii

CHAPTER 1 REVIEW OF CHROMATIN -REMODELING IN PLANTS

1.1 Introduction 1

1.2 Chromatin 3

1.3 Chromatin Remodeling 5

1.4 Chromatin Remodeling Mechanisms 7

1.4.1 ATP-Dependent Chromatin Remodeling 7

1.4.2 ATP-Independent Chromatin Remodeling 10

1.4.3 Histone Acetylation/Deacetylation 13

1.5 Structure Of Histone Acetyltransferases 19 (HATs)/ Histone Deacetylases (HDACs)

1.6 Histone Acetylation And Deacetylation In Plant Development 27

1.7 Glucose Metabolism and Cell Cycle Progression 30

1.8 Glucose and Plant Development 34

1.9 Glucose and Chromatin Remodeling 38

1.10 Glucose Sensing in Plants $\quad 39$

1.11 Glucose and the Plant Hormone ABA 47

1.12 Abscisic Acid and Epigentic Process in Plant Development $\quad 48$ and Stress Responses

1.13 Thesis Objective 51

\section{CHAPTER 2 MATERIALS AND METHOD}

2.1 Plants Materials and Growing Conditions 53

2.2 Identification and Molecular Characterization of 54

Homozygous T-DNA HD2B and HD2D Insertion Lines

2.2.1 Mutants and Transgenic Plants 54

2.2.2 Plant Genomic DNA Extraction and Genotyping 56 
2.2.3 Loss of Function Confirmation by SemiQuantitative Reverse -Transcription PCR (RT-PCR)

2.3 Induction of HD2 Gene Expression upon Glucose Treatment 71

2.4 Transcriptional Compensation by RT-PCR 72

$2.5 \quad H D 2$ and Sugar Signalling $\quad 74$

2.6 GUS Histochemical Staining 75

$\begin{array}{lll}2.7 & \text { GUS Specific Activity } & 75\end{array}$

2.8 Quantification of HD2 Transcript Level upon 77

Soluble Carbohydrate Treatment by Real-Time PCR Method

2.9 Phenotypic Analysis on HD2 Loss-of-Function 79 Lines and Overexpression Line

\section{CHAPTER 3 RESULTS}

3.1 Specificity of $H D 2$ Induction 80

3.2 Glucose Induction of HD2 Family is Specific to 81 the $H D 2 A$ and $H D 2 D$ Members

3.3 HD2 Induction by Glucose is Mediated by Hexokinase- 84 Independent Signaling

3.4 HD2 Family Members are Independently Regulated 90

3.5 Roles of HD2 Family Members in Seed Germination 95

3.6 HD2 function in Post-germinative Development 100

3.6.1 Modulation of HD2 expression by the Plant Hormone 101 ABA

3.6.2 Relationship of Glucose/HD2 and ABA Signal 105 Transduction Pathway

3.6.3 Relationship of the Glucose/HD2 Pathway Relative 113 to Abiotic Stress Pathways

3.7 The HD2C Promoter is Not Responsive to Glucose in 127 Wild-type Background

\section{CHAPTER 4 DISCUSSION AND FUTURE DIRECTION}

Discussion

4.1 Sugars and ABA Signal Transduction Pathway in Embryo Development

4.2 Discovery of a New Glucose Signal Transduction Pathway 139 Mediated by $H D 2$ genes

4.3 Model for the Fine Control of Germination by the Glucose/HD2 pathway 
4.4 Conclusion

APPENDIX

Appendix I Mutant Characterization

Molecular Characterization of $H D 2 B$ and $H D 2 D$

T-DNA Insertion lines

Appendix II Bioinformatics Promoter Analysis

Putative Sugar Motifs Search

151

REFERENCES

156 
Table 1: Identified covalent modifications of amino acids contained within plant histones

Table 2: $\quad$ Subunits of the NURD complexes 16

Table 3: Identified histone acetyl transferases (HAT) 21 within the Arabidopsis thaliana genome

Table 4: Histone deacetylase (HDAC) genes in the Arabidopsis thaliana genome

Table 5: $\quad$ Selected sugar and phytohormone defect responses

Table 6: $\quad H D 2 D$ and $H D 2 B$ mutant lines analyzed for phenotypic abnormalities

Table 7: $\quad$ Genotyping primers for the $H D 2 D$ and $H D 2 B$

T-DNA insertion lines

Table 8: Verification of T-DNA Insertion Using LB and RP/LP Primers

Table 9: Detection of full length and short length mRNA

Table 10: $\quad$ Primers used for Real-Time PCR 
Figure 1: Three glucose sensing and signal transduction pathway

Figure 2: Glucose sensing in yeast $\quad 45$

Figure 3: Heterotrimeric G-protein modes of action 46

Figure 4: $\quad$ Map of HD2B T-DNA insertion lines and 63

Figure 5: Map of HD2D T-DNA Insertion lines and designated 66 primers for truncated mRNA and full length mRNA detection

Figure 6: Quantification of soluble carbohydrate induction of the HD2 family transcripts by Real-Time PCR in Columbia-0

Figure 7: Glucose insensitive-2 (gin2-1) phenotype

Figure 8: $\quad$ RT-PCR analysis of $H D 2$ induction by $3 \%(w / v)$ glucose in Ler (wt) and gin2-1 null mutant

Figure 9: $\quad$ RT-PCR analysis of $H D 2$ induction by soluble sugars in gin2-1 null mutant and Ler wild-type control

Figure 10: RT-PCR analysis of HD2 mutant lines to detect upregulation of other $H D 2$ genes in the absence of one or two functioning $H D 2$ genes upon $3 \%$ glucose

Figure 11: RT-PCR analysis of $H D 2$ induction by $3 \%(w / v)$ glucose in the 35S::HD2C-GFP and wild-type Col-0

Figure 12: Seed germination analysis

Figure 13: Glucose effect on seed germination and root length 98

Figure 14: RT-PCR analysis of $H D 2$ repression by $100 \mu \mathrm{m}$ ABA 103

Figure 15: RT-PCR analysis of $H D 2$ and ABA biosynthesis gene 104 expression over 16 hours of $3 \%(\mathrm{w} / \mathrm{v})$ glucose application 
Figure 16: Phenotypic analysis of 35S::HD2A-GFP, hd2a, hd2c, and $h d 2 a h d 2 c$ (double mutants (D.M)) under osmotic stress conditions (ABA)

Figure 17: Root phenotypic analysis of $35 S:: H D 2 A-G F P, h d 2 a, h d 2 c$, and $h d 2 a$ hd2c (double mutants (D.M)) under osmotic stress conditions (ABA)

Figure 18: Phenotypic analysis of 35S::HD2A-GFP, hd2a, hd2c, and $h d 2 a h d 2 c$ (double mutants (D.M)) under abiotic stress conditions $(\mathrm{NaCl})$

Figure 19: Root phenotypic analysis of 35S::HD2A-GFP, hd2a, hd2c, and $h d 2 a$ hd2c (double mutants (D.M)) under abiotic stress conditions $(\mathrm{NaCl})$

Figure 20: Phenotypic analysis of 35S::HD2A-GFP, hd2a, hd2c, and $h d 2 a h d 2 c$ (double mutants (D.M)) under abiotic stress conditions (mannitol)

Figure 21: Root phenotypic analysis of 35S::HD2A-GFP, hd2a, 124 $h d 2 c$, and $h d 2 a h d 2 c$ (double mutants (D.M)) under osmotic stress conditions (mannitol)

Figure 22: Histochemical GUS staining of AtHD2C::GUS transgenic plants

Figure 23: Quantitative analysis of GUS specific activity in 130 AtHD2C:: GUS transgenic plants

Figure 24: New proposed model of $H D 2$ function

Figure 25: $\quad H D 2 D$ mutant genotyping and RT-PCR analysis

Figure 26: Promoter sequence search of telo-box motifs in $H D 2 A$ 153 and $H D 2 D$ promoters 


\section{LIST OF ABBREVIATIONS}

ABA: Abscisic acid

ACF: Chromatin Assembly and Remodeling Factor

AtRBR1: Retino-Blastoma-Related Protein1

BBM: BABYBOOM

CAB: Chlorophyl a/b-binding protein

CDKs: Cyclin Dependent Kinases

CHRAC: Chromatin Accessibility Complex

CHS: Chalcone Synthase

CIN1: Cell Wall Invertase 1

EFS: Early Flowering In Short Days

ELF7: Early Flowering7

FLC: Flowering Locus C

FLD: Flowering Locus D

FRI: FRIGIDA

GCN5: General Control Non-Derepressible 5

HATs: Histone Acetyltransferases

HD2: Histone Deacetylases 2

HDA1: Histone Deacetylase A1

HDA19: Histone Deacetlase A19

HDACs: Histone Decetylases

HXK: Hexokinase

KRP: Kip-related proteins 
NURF: Nucleosome-Remodeling Factor

3-OMG: 3-O-methyl-D-glucose

PAF1: RNA Polymerase Factor1

PC: Plastocyanin

PIE1: Photoperiod-Independent Early Flowering1

PKL: PICKLE

RBCS: Ribulose-1, 5-bisphosphate carboxylase

RLT: Lysis Buffer

RPD3: Reduced Potassium Deficiency 3

RPE: Wash Buffer with ethanol

RW1: Wash Buffer

SIR2: Silent Information Regulator2

SRC1: Steroid Receptor Coactivator 1

TAF॥ 250: TATA Binding Protein Associated Factor II 250

TSA: Trichostatin A

VIP6: Vernalization Independence6 


\section{ABSTRACT}

Histone deacetylases (HDACs) are an essential class of enzyme that are required by eukaryotic organisms for the coordinated expression of genes. They therefore play key roles in cell differentiation and organ development through global gene regulation. The HD2 sub-family of HDACs are unique to the plant kingdom. Arabidopsis thaliana contains four members of the HD2 family known as $H D 2 A, H D 2 B, H D 2 C$, and $H D 2 D$. A prior thesis by Adam Colville identified the induction of $H D 2$ transcripts in response to glucose, sucrose and fructose.

Using quantitative real-time PCR, the results of my study indicate that the induction of $H D 2$ expression by glucose, sucrose and fructose is specific to the $H D 2 A$ and $H D 2 D$ members in the wild-type background. Using a hexokinase null mutant in the signaling, gin2-1, the glucose-HD2 induction pathway was found to be mediated by a hexokinase-independent pathway. The induction of $H D 2 A$ and $H D 2 D$ by glucose may occur directly at the promoter through motifs that have been identified previously in the promoters of glucose responsive genes.

Phenotypic analysis of $h d 2$ mutants suggested functional specialization among the HD2 members in embryo development and seed germination. HD2A and $H D 2 C$ play opposite roles in the control of seed germination.

This study reveals functional differences among the HD2 family members, and provides new insights into the mechanisms that govern control of HD2 gene expression and the role that this novel gene family performs in plant development. 


\section{THESIS SUMMARY}

The goal of this investigation was to understand the role of the HD2 plant specific class of histone deacetylases during plant development. The HD2 proteins were discovered to be a class of enzymes unique to the plant kingdom and were previously hypothesized to be implicated in transcriptional repression during embryogenesis through chromatin remodeling (Wu et al., 2000b). Chromatin remodeling is a process that alters histone-DNA interactions resulting in the activation or repression of target genes.

Chapter one of this investigation summarizes the existing literature on chromatin-remodeling and chromatin modification processes. Examples of plant research are used to illustrate chromatin remodeling processes. The role of chromatin modification especially acetylation and deacetylation during plant development is reviewed. In addition, the role of glucose and the plant stress hormone abscisic acid in plant development and chromatin remodeling is also reviewed.

Chapter two focuses on experiments designed to understand the role of the HD2 histone deacetylases in plant development in the model organism Arabidopsis thaliana. Over-expression and loss-of-function mutant lines were analyzed for phenotypic abnormalitites under various conditions. To understand the developmental role an analysis of publicly available microarray experiments was previously under-taken leading to the finding that HD2 expression was directly linked to the concentration of soluble carbohydrates (glucose, fructose, and sucrose) in seedlings. This finding will be quantified by real-time PCR in this 
investigation, in addition to the characterization of the HD2 expression specificity. A recent study showed that the expression of HD2 class of histone deacetylase is also modulated in response to the plant stress hormone abscisic acid (ABA). Thus, the aim of this study is to investigate the signal transduction pathway of $H D 2$ induction by glucose and identify target genes involved in the ABA signaling pathway. Our results show that the glucose-HD2 pathway is specific to certain members of the $H D 2$ family, $H D 2 A$ and $H D 2 D$. It further shows that the HD2glucose pathway is mediated through a hexokinase - independent pathway. An alternative hypothesis was developed in which HD2 promoter elements respond to glucose directly by specific motifs. The hypothesis will be tested using synthetic promoters with mutations in the sugar responsive elements within the promoter sequences of the HD2 genes. The approach and the findings of these experimental results are discussed in chapter three, four and five respectively. 


\section{CHAPTER 1}

\section{REVIEW OF CHROMATIN - REMODELING IN PLANTS}

\subsection{INTRODUCTION}

Chromatin remodeling is used to describe the reconfiguration of proteinDNA interactions that convey changes in activities such as gene expression, repression or recombination (Aalfs and Kingston, 2000). The process of chromatin remodeling may be ATP-dependent, ATP-independent or combine both processes. It involves histone deacetylase activity which regulates gene expression by controlling the level of acetylation of the amino-terminal domains of histones. Chromatin is remodeled in response to alterations in plant physiology. The ability to detect physiological stimuli and transfer the proper response to chromatin is critical to all cellular functions. Distinct signal transduction pathways may be activated that induce or repress specific sets of genes upon receiving external signals. Examples include growth factor stimulation or exposure to stress. The transduction of signals from cell surface to the nucleus may involve phosphorylation cascades that allow quick transmission and amplify the signal through multiple factors and genes that lead to integrated cellular responses such as proliferation, differentiation, or apoptosis (Cheung et al., 2000).

Among the various regulatory systems and signals in plants, carbonmetabolite-mediated gene regulation is important (Farrar, 1991; Sheen, 1994; Koch, 1996; Jang and Sheen, 1997; Smeekens and Rook, 1997). Sugars appear to control many plant genes and play pivotal roles in diverse processes such as 
photosynthesis, protein storage, or starch, lipid, and nitrogen metabolism (Hattori et al., 1990, 1991; Nakamura et al., 1991; Karrer and Rodriguez, 1992; Krapp et al., 1993; McLaughlin and Smith, 1994; Chevalier et al., 1996). Generally, high sugar concentrations are associated with repression of genes involved in photosynthesis and the mobilization of starch, lipid, and protein storage and the induction of genes required for storing carbon metabolites. The understanding of sugar regulation of gene expression in plants is limited; however, a significant amount of information is available from research on bacteria (Magasanik, 1961; Ullmann, 1985; Saier, 1989) and yeast (Gancedo, 1992; Johnston and Carlson, 1992; Trumbly, 1992; Thevelein, 1994; Ronne, 1995). In yeast, glucoserepressible genes are regulated by the enzyme hexokinase (HXK). The enzyme phosphorylates glucose to glucose-6-phosphate, initiating a signal that is perceived by the GLC7 complex (the catalytic subunit of protein phosphatase type1 (PP1)) (Tu and Carlson, 1995). This results in the activation of the down stream SSN6/TUP1 corepressor protein complex through the re-modeling of chromatin structure upon binding to the transcription factor MIGI, thus repressing gene expression. In the absence of glucose, a pathway involving several protein complexes, including the SNF2 (catalytic subunit of the SWI/SNF chromatin remodeling complex containing ATPase activity) - containing complex, reverses the SSN6/TUP1- allowing genes to be transcribed. In plants, sugars have a signaling role in which the hexokinase protein is proposed to play a fundamental role (Sheen et al., 1999; Smeekens, 2000; Moore et al., 2003). 
Three different glucose-signalling pathways have been suggested in plants (Sheen et al., 1999). A HXK-independent pathway that regulates genes such as those for chalcone synthase (CHS) and cell wall invertase1 (CIN1). These genes are induced by sugar analogs such as 6-deoxyglucose and 3-Omethyl glucose that are taken up by plant cells but not further metabolized. Such hexose sensing might be mediated by transporter-like receptors and G-proteins such as described in yeast (Ozcan et al., 1996). The two other pathways are HXK dependent, one is glycolysis-dependent and is induced by over-expression of both native Arabidopsis AtHXK1 and heterologous yeast HXK1 in Arabidopsis. The second pathway requires Arabidopsis AtHXK1 and effects photosynthetic genes such as those for chlorophyll a/b-binding protein (CAB), the small subunit of ribulose-1,5-bisphosphate carboxylase (RBCS) and plastocyanin (PC) (Sheen et al., 1999; Moore et al., 2003).

The aim of this investigation is to understand the function of histone deacetylases 2 (HD2) during plant development in relation to chromatin remodeling under modulation of the sugar environment.

\subsection{CHROMATIN}

In eukaryotes, DNA is compacted into chromatin within the nucleus. The basic unit of chromatin is the nucleosome which consists of $145 \mathrm{bp}$ of DNA that is wrapped around an octamer of basic proteins, the core histones. The octamer comprises two of each of the histone molecules $\mathrm{H} 2 \mathrm{~A}, \mathrm{H} 2 \mathrm{~B}, \mathrm{H} 3$ and $\mathrm{H} 4$ (Kornberg and Klug, 1981). Each histone protein contains two distinct functional domains, 
the globular domain and the highly charged $\mathrm{N}$-terminal histone tail. The globular domain which consists of approximately 70 amino acids toward the $C$ termini of the histones mediates interactions between the core histones and between the histones and DNA. The $\mathrm{N}$ termini are not critical to sustain the integrity of nucleosomes. Histone tails have flexible contacts with DNA and adjacent nucleosomes and act as sites for post-translational modification (Ausio et al., 1989).

There have been identified two distinct forms of chromatin, heterochromatin and euchromatin (Heitz, 1928). The two forms were distinguishable by the degree of stain intensity; for example, heterochromatin is a highly condensed region that gives higher staining with DNA binding dyes such as DAPI. It is associated with transcriptional repression, low content of genes, late replication, and a high content of repetitive sequences. Conversely, euchromatin is less compact and yields lighter staining than the heterochromatin. Unlike heterochromatin, euchromatin is associated with active transcription of DNA and a higher content of genes (Heitz, 1928).

Other essential chromatin proteins include DNA topoisomerase II, which comprises the bulk of the metaphase chromosome scaffold (Earnshaw et al., 1985), and the cohesins and condensins, which are implicated in chromosome condensation during cell division and occasionally involved in gene regulation during interphase (Chuang et al., 1994, 1996; Hirano, 1999; Lupo et al., 2001). Other essential chromatin components include remodeling factors (discussed below) and RNA. RNA is an important component of chromatin that is involved in 
gene regulation. For example, $\mathrm{X}$ chromosome inactivation in mammalian cells, dosage compensation in Drosphila and mouse pericentromeric heterochromatin formation all require RNA molecules. Moreover, RNA has been shown to be important in directing de novo methylation in plants (Wassenegger, 2000).

\subsection{CHROMATIN REMODELING}

The structure of chromatin fibers influences transcription, repair, replication, and recombination processes (Tariq and Paszkowski, 2004). The degree of compaction is directly correlated with the accessibility of DNA to DNA binding proteins and the transcription of genes. Strong interactions between DNA and histones result in DNA compaction associated with gene repression, as genes are not accessible for transcription. For transcription and replication to occur, chromatin must unfold to expose the DNA template to transcription factors and other proteins. The change in chromatin structure that converts loci from inaccessible to an accessible conformation may be reversible and is termed "chromatin remodeling". If a set of genes expressed under normal conditions is not sufficient to maintain cellular function, the transcriptome may be re-profiled to allow the survival of the organism. Thus, chromatin remodeling in response to alterations in cell physiology underlies control of the transcriptome (Cairns, 1998) and is a dynamic process. Chromatin remodeling may be an ATP-dependent, ATP-independent or may combine both processes. Enzymes that modify the histones and chromatin-remodeling machines utilizing ATP are the major factors that influence chromatin structure and function (Loidl, 2004). A histone code has 
been hypothesized that describes chemical modifications present on the $\mathrm{N}$ terminus of histone proteins that dictate the accessibility of a genetic locus to the gene expression factors, directly influencing the abundance of mRNA transcript. Post-translational modification of histone tails by acetylation, methylation, phosphorylation, ubiquitination, glycosylation, ADP ribosylation, carbonylation, sumoylation and biotinylation play essential roles in determining chromatin structure, functional rearrangements and are hence essential elements for the complex 'epigentic histone code' (Lodi, 2004). The pattern of post-translation modification comprises a histone code that describes the nature of the surrounding chromatin as either conductive or repressive to transcription (Strahl and Allis, 2000). However, studies have shown that the histone code is complex. Position and modification environment have been shown to play a critical role in the outcome of gene repression or activation. For example, methylation of lysine 9 of histone $H 3$ is associated with silenced promoters on the inactive $X$ chromosome in mammals, while methylation of lysine 4 of histone $H 3$ is found at active genes (Boggs et al., 2002). The same modification could also be associated with different chromatin states; for example phosphorylation of serine 10 of histone $H 3$ can be correlated with both active and condensed chromatin (Cheung et al., 2000). Furthermore, phosphorylation of serine 10 of histone $H 3$ does not seem to be associated with maize chromosome condensation, indicating that the histone code might not be conserved between species (Kaszas and Cande, 2000). However, the order of histone modification contributes to the code. For example, methylation of arginine 3 of histone $\mathrm{H} 4$ 
precede and leads to acetylation of lysine 8 of histone $\mathrm{H} 4$ promoting transcription (Wang et al., 2001). This supports the "histone code" hypothesis in which no single modification is responsible for the alteration of the chromatin structure and that the sum of various covalent modification may result in the up or down regulation of a gene expression.

\subsection{CHROMATIN REMODELING MECHANISMS}

Chromatin-modifying complexes can be classified into three main groups based on their mode of action. Group I is an ATP-dependent complex which uses the energy of ATP hydrolysis to locally disrupt the association of histones with DNA. Group II is ATP-independent chromatin remodeling mechanism that focuses on the N-terminus histone tails to remodel chromatin. Group III is composed of histone acetyltransferase (HA) and histone deacetylase (HDAC) complexes. This group regulates gene expression by controlling the level of acetylation of the amino-terminal domains of histones.

\subsubsection{ATP-DEPENDENT CHROMATIN REMODELING}

Several protein complexes are involved in transcription regulation that function by histone modification (Vignali, et al., 2000). The first step of the proposed mechanism for chromatin remodeling involves the binding of the protein complex to the chromatin either directly or through a transcription factor. Each of the ATP-dependent chromatin-remodeling complexes has an ATPase subunit that is from the SNF2 superfamily of proteins. The ATPase-dependent 
chromatin-remodeling complexes are divided into two major groups based on the identity of the ATPase subunit. The two major groups are: the SWI2/SNF2 group and the SWI (ISWI) group (Vignali, et al., 2000). A third class of ATP-dependent complexes has been recently described that contain a Snd2-like ATPase. The SWI/SNF was the initial remodelling complex isolated from yeast and found to contain 11 highly conserved ATPase subunits. The complexes were found to modify the interaction between DNA and histones to allow access of transcription factors which may suppress or promote gene expression (Vignali, et al., 2000). Studies of SWI/SNF2 -like proteins in Arabidopsis thaliana have shown that the SWI/SNF2 complex function in DNA repair mechanisms (Shaked et al., 2006) as well as the transition to floral development (Wagner and Meyerowitz, 2002).

The ISWI complex is the second kind of ATP-dependent remodelling complex. This group contains the ATP-utilizing chromatin assembly and remodelling factor (ACF) (Ito et al., 1997), nucleosome-remodeling factor (NURF) (Tsukiyama and Wu, 1995), and chromatin accessibility complex (CHRAC) (Varga-Weisz et al., 1997) which were originally recognized in Drosophila melanogaster, and contain the nucleosome-dependent ATPase ISWI (Vignali, et al., 2000). Complexes of ISWI are smaller and contain fewer subunits than their SWI/SNF counterpart. The nucleosome-remodeling factor (NURF) has a molecular mass of $500 \mathrm{kDa}$. It contains four subunits, ISWI, p215, and the WD repeat protein Nurf-55 that is identical to the $55 \mathrm{kDa}$ subunit of the dCAF-1 assembly factor found in Drosophila chromatin (Vignali, et al., 2000). The chromatin accessibility complex (CHRAC) has a molecular mass of $670 \mathrm{kDa}$ and 
five subunits. Two of these subunits were acknowledged as ATPases, ISWI and topoisomerase II (Vignali, et al., 2000). The ACF group contains ISWI which is the catalytic subunit and has a molecular mass of about 22-kDa. Recent research has identified a protein as a component of ACF. This protein is known as Acf1 and was purified from Drosophila. Acf1 was shown to exist as two complexes that contain ISWI and one of the two Acf1 forms, $\mathrm{p} 170$ or p185. It is understood that ACF exists as heterodimers of either Acf1 form or ISWI form since its molecular mass is only $220 \mathrm{kDa}$ (Vignali, et al., 2000).

The third group of the ATPase-dependent chromatin-remodeling complexes is a chromatin-remodeling and deacetylase complexe and is termed the Mi-2 group (Vignali, et al., 2000). Analogous complexes that contain chromatin-remodeling and deacetylase activities were purified from human cells. These complexes were identified as NURD, NURD, and NRD. The hNURD complex includes the HDACs, HDAC1 and HDAC2, the retinoblastoma protein (Rb)-associated proteins, RbAp46 and RbAp48, and the Swi2/Snf2 ATPase homologue $\mathrm{CHD} 4$, that is also known as $\mathrm{Mi}-2^{3}$. This group of complexes utilizes the two mechanisms: ATP-dependent and ATP-independent chromatin remodelling that occurs through covalent modification of histone tails. The two processes are intricately linked in vivo as one mechanism might be essential to recruit ATP-dependent chromatin-remodelling complexes to a locus or the cell could combine both processes into a single protein complex as it is the case with the Mi-2 complex (Wade et al., 1998, Zhang et al., 1998, Xue et al., 1998, Tong et al., 1998). 


\subsubsection{ATP -INDEPENDENT CHROMATIN REMODELING}

ATP-independent chromatin remodelling studies focus on the $\mathrm{N}$-terminus histone tails that extend from the nucleosome since it is considered to play an essential role in the conformational remodeling of a locus. It is believed that the tails play an important role in mediating the strength of DNA/protein interaction by reducing the net charge on the tail. This could prevent access of transcription factors to the gene through steric hindrance or provide a signal to engage chromatin remodelling factors (Wagner, 2003). Residues in the histone tails may be subjected to a diverse array of post-translational modifications such as acetylation, methylation, phosphorylation, and ubiquitination. There is no single covalent modification that is responsible for the alteration of chromatin structure, but rather, it is the sum of several covalent modifications that may result in the regulation of a gene expression. Table 1 lists known covalent modifications. 
Table 1: Covalent modifications of amino acids contained within plant histones.

\begin{tabular}{|c|c|c|c|}
\hline Histone & $\begin{array}{l}\text { Amino Acid } \\
\text { Residue }\end{array}$ & Modification State & $\begin{array}{l}\text { Gene } \\
\text { Expression/Repression }\end{array}$ \\
\hline \multirow[t]{4}{*}{$H 2 A$} & Ser1 & Phosphorylation & - \\
\hline & Lys5 & Acetylation & - \\
\hline & Lys9 & Acetylation & - \\
\hline & Lys 119 & Ubiquitination & - \\
\hline \multirow[t]{6}{*}{$H 2 B$} & Lys5 & Acetylation & - \\
\hline & Lys12 & Acetylation & - \\
\hline & Ser14 & Phosphorylation & - \\
\hline & Lys15 & Acetylation & - \\
\hline & Lys20 & Acetylation & - \\
\hline & Lys120 & Ubiquitination & - \\
\hline \multirow[t]{10}{*}{$H 3$} & Arg2 & Methylation & - \\
\hline & Lys4 & Methylation & Gene expression \\
\hline & Lys9 & Methylation & Gene Repression \\
\hline & Lys9 & Acetylation & - \\
\hline & Ser10 & Phosphorylation & - \\
\hline & Lys14 & Methylation & - \\
\hline & Arg17 & Methylation & - \\
\hline & Lys 18 & Methylation & - \\
\hline & Lys18 & Acetylation & - \\
\hline & Lys23 & Methylation & - \\
\hline
\end{tabular}


Continue table 1

\begin{tabular}{llll}
\hline Lys23 & Acetylation & - \\
Arg26 & Methylation & - \\
Lys27 & Methylation & - \\
Ser28 & Phosphorylation & - \\
Lys36 & Methylation & - \\
Lys79 & Methylation & - \\
Arg3 & Methylation & - \\
Lys5 & Acetylation & - \\
Lys8 & Acetylation & - \\
Lys12 & Acetylation & - \\
Lys16 & Acetylation & - \\
Lys20 & Methylation & - \\
Lys20 & Acetylation & -
\end{tabular}


Covalent modification of histone proteins and its alteration of gene expression offer a method for regulating transient gene expression that is required for tissue differentiation during growth and development. The cell could quickly change the expression of gene targets through the reversal of the modification by enzymatic complexes such as deactylases, and demethylases, or through dilution of the modification during replication of the genetic material throughout division. This may be important in developmental pathways, where the transduction of the signal leads to the activation of multiple factors and genes for tissue proliferation and differentiation during growth and development.

\subsubsection{HISTONE ACETYLATION/DEACETYLATION}

The third group of chromatin-modifying complexes is composed of histone acetyltransferase (HAT) and histone deacetylase (HDAC). Acetylation of histones is usually related to transcription activity, while deacetylation of histones is related to transcription silencing (Zhou et al., 2004). The level of acetylation depends on the competing activities of both histone acetyltransferases (HATs) and histone deaceylases (HDACs) enzymes (Zhou, et al., 2004).

Although the mechanism of how acetylation modification modulates geneexpression is not clear, acetylation of the $\varepsilon$-amino groups of lysine near the amino termini of core histone proteins is proposed to neutralize the positively charged histone octamer. This results in weakening the interaction of the histone octamer with the negatively charged DNA provoking an open chromatin conformation that allows the access of transcription machinery to promoters 
resulting in the expression of a target gene. Another mechanism of how acetylation of the histone tail might lead to chromatin remodelling and gene expression is through the transfer of the acetyl moiety as a signal that result in the recruitment of ATP-dependent chromatin remodelling complexes to the locus. Thus, the role of histone acetylation could be to direct the binding of nonhistone proteins. Acetylation of histone $\mathrm{H} 3$ could lead to a $10-30 \%$ reduction of positive charge which is not likely to have an effect on interactions with DNA (Peterson and Laniel, 2004); for example, acetylation of Lysine 8 within histone H4 promotes the recruitment of the ATP-dependent chromatin remodelling enzyme, human SWI/SNF- by a bromodomain within the Brg1 subunit. The main role of the bromodomain, and the chromodomain is to serve as a position for assembly of co-activator vs. co-repressor complexes.

A last proposed mechanism is that acetyl groups loosen the DNA/protein interaction by steric hindrance. The deacetylases may remove the acetyl groups from specific lysine residues thus restoring positive charges on the lysine residues and strengthening the interaction between the positively charged lysine and the negatively charged DNA. This may restrict access of the transcriptional regulatory to the promoter and down regulates gene expression (Tian and Chen, 2000).

Histone acetyltransferases and histone deacetylases operate together to coordinate gene expression within the cell. However, studies have shown that both HATs and HDACs lack specificity and require partner proteins to target them to a specific locus. Histone acetyltransferases were found to be a part of 
multiprotein complexes, in which their specificity differ based on their partner protein (Lusser et al., 2001). Histone deacetylases have been also found to be a part of multisubunit complexes. An example where HDACs interact with other proteins to alter chromatin structure and result in transcription repression is the case of Maize in which AtRPD3 interacts with a retinoblastoma-related (ZmRBR1) homologue to silence a particular locus (Rossi et al., 2003). Research has identified three similar acidic polypeptides ( $p 39, p 42$, and $p 45)$ within the HD2 maize protein. The p42 and p45 proteins were identified as interacting subunits of the deacetylase complex in Maize (Lusser et al., 1997).

The identification of the vertebrate Mi2-Nurd complex has connected covalent modification of histones such as deacetylation with ATP-dependent chromatin remodeling mechanism. The Mi2-Nurd complex has been identified to contain both histone deacetylase activity and a SNF-related ATPase (Wade et al., 1998, Zhang et al., 1998, Xue et al., 1998), thus combining both processes to regulate gene expression. Homologues to the Mi2-Nurd complex were identified in humans and plants (Zhang et al., 1998). Subunits identified in the human and plants NuRD complex are listed in table 2. 
Table 2: Subunits of the NURD complexes

Organism Subunit of NuRD HDACs Component

\begin{tabular}{|c|c|c|}
\hline Human NRD & 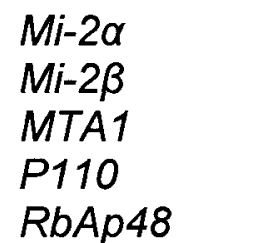 & HDAC1/HDAC2 \\
\hline Human NURD & $\begin{array}{l}M i-2 \beta \\
M T A 1 \\
R b A p 48 / p 46\end{array}$ & HDAC1/HDAC2 \\
\hline Human $N u R D$ & 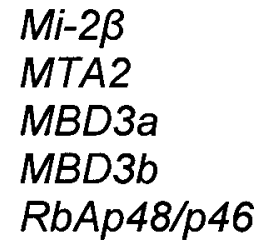 & $H D A C 1 / H D A C 2$ \\
\hline Xenopus laevis MI-2 & $\begin{array}{l}\text { Mi-2 } \\
\text { MTA1-like } \\
\text { P66 } \\
\text { PrZp48/p46 } \\
\text { MBD3 } \\
\text { MDB3LF }\end{array}$ & $R P D 3$ \\
\hline
\end{tabular}


The discovery of a plant homologue of Mi-2, PICKLE (PKL), linked chromatin remodeling complexes with development (Ogas et al., 1999). PKL mutant plants expressed embryonic traits post-germination suggesting that $P K L$ is required for repression of the embryonic developmental program and thus plays a role in chromatin remodeling and development. Furthermore, disruption and silencing of HISTONE DEACETYLASE19 (HDA19, AtRPD3A) and AtHD2A together resulted in arrested growth after germination, the expression of somatic embryos, and the expression of embryo marker genes (Tanaka et al., 2008), indicating that patterns of normal processes of acetylation and deacetylation are essential for normal plant development.

Another example of a gene in Arabidopsis that is partially controlled by the level of acetylation is the FLOWERING LOCUS $C$ (FLC). The switch from vegetative growth to reproductive development requires reprogramming of gene expression. This reprogramming is controlled by general chromatin remodellers because inactivation of several putative chromatin factors promotes early or late flowering phenotypes. FLC encodes a MADS-box transcription factor that blocks the floral transition. FLC expression is controlled by two pathways; the autonomous pathway which activates flowering and the vernalization pathway which provokes flowering after a prolonged exposure to cold temperatures (Reyes, 2006).

A mutational analysis and chromatin immunoprecipitation data revealed that chromatin at the FLC locus chromatin is able to exist in three functional states and a couple of intermediate states. $F L C$ is found in the inactive state in 
the presence of either dominant alleles of FRIGIDA (FRI) or mutants of the autonomous flowering pathway (Reyes, 2006). Histone $\mathrm{H} 3$ and $\mathrm{H} 4$ in the FLC promoter and the first intron have a high level of acetylation and H3K4 trimethylation are epigenetic marks of active chromatin. Histone $\mathrm{H} 3 \mathrm{~K} 4$ trimethylation is promoted by an RNA POLYMERASE FACTOR1 (PAF1)-like complex and by a putative histone methyltransferase called EARLY FLOWERING IN SHORT DAYS (EFS) (Reyes, 2006). The PAF1 complex consists of EARLY FLOWERING7 (ELF7), ELF8, VERNALIZATION (VIP6), VIP3, VIP4 and VIP5. Moreover, a high level of FLC activation also requires an ATPdependent remodeller of the SNF2 family known as PHOTOPERIODINDEPENDENT EARLY FLOWERING1 (PIE1). Analysis of the SNF2 family in Arabidopsis show that PIEI is most similar to S. cerevisiae SWR1 (Swi2/Snf2related ATPase), which is a remodeller involved in histone $\mathrm{H} 2 \mathrm{~A}$ replacement. This raises the possibility that nucleosomes at the $F L C$ locus might contain a specific histone H2A variant when the gene is entirely active (Reviewed in Reyes, 2006). The FLC gene is repressed in the absence of a strong FRI allele by the autonomous pathway. In this repressed, inactive state, histones at the FLC promoter and first intron are deacetylated and histone H3K4 is demethylated. Furthermore, two proteins of the autonomous pathway were found to be homologs of proteins in mammalian histone deacetylase complexes. FVE is a histone chaperone of the MSI family while FLOWERING LOCUS D (FLD) is homolog of the human histone H3K4 demethylase LSD1(LY-CIFC HISTONESPE-CIFIC HISTONE DEMETHYLASE1). Moreover, another protein appears to 
play a role in sustaining the repressed state in RELATIVE OF EARLY FLOWERING 6 (REF6). The REF6 protein jmjC and jmjN domains. These two domains function in histone hydroxylation or histone demethylation. After vernalization, $F L C$ can also exist in a silenced heterochromatinised state. In comparison to other states, this chromatin configuration is epigenetically inherited through mitosis. In this state, the action of VRNP Polycomb complex results in the deacetylation of histones $\mathrm{H} 3$ and $\mathrm{H} 4$, and the methylation of histone $\mathrm{H} 3$ in residues $\mathrm{K} 9$ and $\mathrm{K} 27$. Moreover, two other proteins known as VRN1 and VERNALIZATION INSENSITIVE 3 (VIN3), were found to be involved in setting this chromatin state (Reviewed in Reyes, 2006).

\subsection{STRUCTURE OF HISTONE ACETYLTRANSFERASES (HATS)/HISTONE DEACETYLASES (HDACS)}

There exist two categories of HATs depending on their subcellular distribution. These are type B HATs and type A HATs (Reyes, 2002). Type B HATs are cytoplasmic complexes involved in acetylation of histone $\mathrm{H} 4$ at positions 5 and 12 prior to its integration into nucleosomes. Type B HATS in Maize (Zea mays) form a heterodimeric nuclear complex. Research in yeast showed that the gene for the $50-\mathrm{kD}$ enzymatic subunit is homologous to yeast HAT1 while the $45-\mathrm{kD}$ subunit is related to the mammalian $\mathrm{Rb}$-associated protein $(\mathrm{RbAp})$ and yeast/plant MSI1 proteins that are found in other chromatinremodeling complexes as well (Lusser et al., 2001). 
Type A HATs are divided into four classes, each with different specificities. The four classes are: the GCN5 (general control non-derepressible 5); and MYST (for "Moz, Ybf2/Sas3, Sas2 and Tip60) family of proteins, CBP/p300 the general transcription factor HATs, which include the TAFII 250 subunit; and the nuclear hormone-related HATs SRC1 (steroid receptor coactivator 1) and ACTR (activator of retinoid receptor) (Lusser, et al., 2001). The Arabidopsis thaliana genome contains 5 members of the GCN5-related N-terminal acetyltransferases, 5 members of the CBP, and 2 members of the TATA BINDING PROTEINASSOCIATED FACTORS (TAF ॥ 250) families. Table 3 illustrates the identified HATs within the Arabidopsis thaliana genome (URL http://www.chromdb.org). 
Table 3: Identified histone acetyl transferases (HAT) within the Arabidopsis thaliana genome.

HAT Family Subfamily Gene Name

GNAT-MYST

CBP

TAFII250
GCN5

ELP3

HAT1

MYST

AtHAC4

AtHAC5

AtHAC12

AtHAF1

AtHAF2 
Sequence homology is present among members of each HAT family, but not between different families. For example, members of the GNAT family contain a conserved HAT domain with 4 conserved motifs termed A, B, C, and D. The GNAT family contains two domains, an N-terminal domain, and a C-terminal catalytic HAT domain. The two domains are divided by a hydrophobic cleft. The histone binds within the A and B motifs of the HAT. The enzyme catalyzes the transfer of an acetyl moiety from acetyl-CoA to the $\varepsilon$-amino group of a lysine residue in the histone tail. These proteins contain a specific histone code through their specialized bromodomain which has been hypothesized to mediate protein to protein interaction such as its interaction between histone tail modification and specific domain of ATP dependent chromatin remodelling complex. The CBPS family share sequence similarity with the $\mathrm{A}, \mathrm{B}$, and $\mathrm{D}$ motifs of the GNAT family. The plant CBPs structure lacks a bromodomain and KIX domain, and contains one zing finger motif. The Arabidopsis thaliana's TAF/I 250 HATs contain a HAT catalytic domain, a C-terminal bromodomain and a zinc finger motif.

Moreover, sequence similarities allowed the identification of 18 HDACs within the Arabidopsis thaliana genome (URL, http://www.chromdb.org). Ten of the 18 HDACs genes were found to belong to the REDUCED POTASSIUM DEFICIENCY3 (RPD3)/HISTONE DEACETYLASE A1 (HDA1) family initially identified in yeast (Rundlett et al., 1996). Two belong to the SILENT INFORMATION REGULATOR2 (SIR2) family initially identified in yeast and four belong to the HISTONE DEACETYLASE2 (HD2) family initially identified in 
maize and are specific to plants (Lusser et al., 1997). Table 4 represents the HDAC genes that have been identified within Arabidopsis thaliana's genome. 
Table 4: Histone deacetylase (HDAC) genes in the Arabidopsis thaliana genome

\begin{tabular}{|c|c|c|c|}
\hline HDAC & Gene & & \\
\hline Family & Name & AGI code & Synonyms \\
\hline & $H D A 2$ & At5g26040 & \\
\hline & HDA5 & At5g61060 & \\
\hline & HDA6 & At5g63110 & AtRPD3B \\
\hline & $H D A 7$ & At5g35600 & \\
\hline & $H D A 8$ & At1g08460 & \\
\hline & HDA9 & At3g44660 & \\
\hline HDA1/RPD3 & HDA10 & At3g44660 & \\
\hline & HDA14 & At4g33470 & \\
\hline & HDA15 & At3g18520 & \\
\hline & HDA17 & At3g44490 & \\
\hline & HDA18 & At5g61070 & \\
\hline & HDA19 & At4g38130 & HDA1, HD1, AtRPD3A \\
\hline SIR2 & HDA12 & At5g55760 & \\
\hline & HDA16 & At5g09230 & \\
\hline$H D 2$ & HDT1 & At3g44750 & $H D A 3, A t H D 2 A$ \\
\hline & HDT2 & At5g22650 & $H D A 4, A t H D 2 B$ \\
\hline & HDT3 & At5g03740 & $H D A 11, A t H D 2 C$ \\
\hline & HDT4 & At2g27840 & $H D A 13, A t H D 2 D$ \\
\hline
\end{tabular}


The HDA1/RPD3 family possesses an $\mathrm{N}$-terminal catalytic domain and a short variable C-terminal domain. The SIR2 family does not share any sequence homology to any of the other histone deacetylases. Moreover, it requires NAD as cofactor for enzymatic function.

The fourth class of HDAC is unique to the plant kingdom. The Arabidopsis genome contains four $H D 2$ members. These are $H D 2 A, H D 2 B, H D 2 C$, and HD2D (Dangl et al., 2001; Pandey et al., 2002; Wu et al., 2003). The discovery of this specific histone deacetylase family in plants raised the possibility of functional specialization. HD2 family have members been found to contain stretches of acidic amino acids characteristic of nuclear proteins. Studies characterized an extended acidic domain from the cDNA sequence (Lusser et al., 1997). Two additional genes, $A t H D 2 A$ and $A t H D 2 B$ were identified from the EST database within Arabidopsis thaliana using the sequence of the identified Maize HD2. AtHD2A is found on chromosome III and AtHD2B is on chromosome V, the two proteins share 44 and $46 \%$ amino acid similarity respectively to maize $H D 2$. Two other related genes named $A t H D 2 C$ and $A t H D 2 D$ were identified through PCR amplification of a cDNA library using a conserved primer sequence. $A t H D 2 C$ is on chromosome $\mathrm{V}$, and $A t H D 2 D$ was found on chromosome II. These genes were found to share sequence similarity with earlier identified HD2 genes. AtHD2C encodes a peptide of 295 amino acids and AtHD2D encodes a peptide of 204 amino acids, each containing eight exons (Dangl et al., 2001).

Further search using the PSI-BLAST program showed significant sequence similarity to an insect protein known as FKBP family peptidyl-proyl cis 
-trans isomerases (PPlases) and to trypanosomal RNA binding protein. The conserved region includes an $\mathrm{NH} 2$ - terminal domain in each of these proteins (Aravind, 1998). Subsequent search of the nonredundant database and the database of the Expressed Sequence Tags (ESTs) with these sequences characterized a new family that contain proteins from plants, yeast, and two parasitic apicomplexans, Toxoplasma gondii and Cryptosporidium parvum (Aravind,1998). Examination of the HD2 family alignment showed a number of conserved hydrophobic positions and polar residues, specifically, an invariant aspartic acid and a histidine that is replaced by an arginine in the trypanosomal RNA-binding protein Nopp44/46 and in the yeast FKBP (Aravind, 1998). It seems that the invariant aspartic acid is the nucleophile that is involved in lysine deacetylation. The conserved histidine (arginine) is thought to facilitate lysine deacetylation by aspartic acid (Aravind, 1998).

The first member of the HD2 family was initially purified from mazie chromatin as a high-molecular weight complex that is made of three nearly identical acidic polypeptides (Lusser et al., 1997). The active enzyme is a phosphoprotein. Thus, it could be subject to post-translational control through phosphorylation pathways. The maize HD2 was found in the nucleolus strongly bound to chromatin, and shares homology with other nucleolar proteins (Lusser et al., 1997). Subsequent studies revealed the structure of this specific class of HDAC. The HD2 class was identified to contain three conserved domains: the $\mathrm{N}$ terminal deacetylase domain, an acidic region that is common in all four of the HD2 family, and a zinc finger motif found in AtHD2A and AtHD2C (Wu et al., 
2000 and Dangl et al., 2001). The zinc finger domain is believed to mediate protein-protein interactions and thus could be involved in protein complex formation. Moreover, phylogenetic analyses have showed that $H D 2 A, H D 2 B$, and $H D 2 C$ are more closely related than $H D 2 D$. Furthermore, $H D 2 A, H D 2 B$, and $H D 2 C$ were found to have a similar expression pattern wherease $H D 2 D$ showed a more unique expression pattern (Zhou et al., 2004). Thus, it has been suggested that the $H D 2 D$ member might have a different, specific function within Arabidopsis thaliana.

\subsection{HISTONE ACETYLATION AND DEACETYLATION IN PLANT DEVELOPMENT}

Histone acetylation and deactylation are fundamental processes required for plant development. Acetylation functions in chromatin remodelling for the quick transition from the repressed to active state. The removal of acetyl groups by histone deacetylases may quickly inhibit transcription when fine control of expression is required. The fine control over expression is due to the enzymes responsible for the addition and removal of groups and specific factors employed for targeting. Acetylation and deacetylation may play essential roles in the coordinated expression of genes and cell differentiation and organ development. Experiments with trichostain A (TSA), a chemical inhibitor of histone deacetylases, resulted in the disruption of histone deacetylation and the silencing of genes HISTONE DEACETYLASE19 (HDA19, AtRPD3A) and AtHD2A 
indicating that normal processes of acetylation and deacetylation are essential for normal plant development (Xu et al., 2005; Wu et al., 2000a; Tian and Chen, 2001; Wu et al., 2000b; Yoshida et al., 1990). Xu et al., 2005 noted that TSA exposure resulted in changes in cellular patterning of roots. Moreover, $\mathrm{Xu}$ et al. (2005) noted hyperacetylation of core histones within one hour of exposure and an increase in the number of root hairs in a TSA concentration dependent manner.

The role of AtRPD3A (AtHD1, HDA19) in development was also demonstrated by gene silencing. The gene coding for AtRPD $3 A$ was introduced in antisense orientation driven by the constitutive -394tCUP promoter. Expression of the antisense transcript resulted in the reduction of AtRPD3A endogenous transcript levels which leads to the delay in the reproductive phase (Wu et al., 2000a). AtRPD3A was also cloned and introduced in antisense orientation driven by the $35 \mathrm{~S}$ constitutive promoter (cauliflower mosaic virus) (Tian and Chen, 2001). Early senescence, reactivation of silenced genes in a non-specific fashion, loss of apical dominance, heterochronic shift to juvenility, homeotic transformation of tissue, defective flowers, and sterility were noted. This indicated that the deacetylase gene, and thus the process of deacetylation, may play a role in numerous developmental processes (Tian and Chen, 2001). Both studies showed that silencing of the deacetylase gene resulted in plants with abnormal primary leaves, which form during early development indicating that deacetylation plays a role in embryo meristem development. 
The HD2 family has also been found to be implicated in embryogenesis and plant development. Previous work on the HD2 gene expression patterns detected the expression of $H D 2 A, H D 2 B$, and $H D 2 C$ in leaves, roots, stems, young plantlets, flower, and siliques with the highest levels being detected within stems, flowers and young siliques followed by seedlings (Zhou et al., 2004). In situ hybridization was also performed, detecting strong expression levels of mRNA in ovules, embryos, shoot apical meristems, and primary leaves. Investigation of the HD2 family by in situ hybridization on embryonic tissue generated through the expression of the Brassica napus transcription factor $B A B Y B O O M(B B M)$ showed a high level of $A t H D 2 A$ and $A t H D 2 B$ induction within the developing embryo but lower concentration in the surrounding tissues. These expression patterns are consistent with their proposed roles in embryogenesis and plant development anticipated through phenotypic analysis using antisense suppression and over-expression lines (Wu et al., 2000). Silencing of AtHD2A expression resulted in aborted seed development in transgenic plants while overexpression of $35 S:: G F P-H D 2 A$ in transgenic Arabidopsis plants generated pleiotropic developmental abnormalities, including abnormal leaves, delayed flowering, and aborted seed development (Wu et al., 2000; Zhou et al., 2004).

$A t H D 2 B$ and $A t H D 2 C$ were found to repress gene expression when targeted to a promoter used to drive reporter gene expression (Wu et al., 2003). These genes likely function in association with transcription factors to repress specific gene targets and thus assist the coordination of gene expression during development. Due to the similarity of gene expression patterns, functional 
redundancy of these genes was suggested. Knockout lines of single $H D 2 A$ and HD2C genes did not show any observable phenotype under normal growth conditions, while double mutants of HD2A HD2C showed an aborted seed phenotype (Colville, 2007). Conversely, the gene expression pattern analysis of the $H D 2 D$ member revealed transcript in stems, flowers, and young siliques, indicating a possible different development role for this gene.

Characterization of the nucleolar matrix of $A$. thaliana was investigated by Calikowski et al., 2003. A protein with sequence similarity to AtHD2A was isolated from the nucleoli and identified by mass spectrometry. Nuclear localization of HD2A was confirmed by Zhou et al., 2004 through microscopic analysis of GFP-AtHD2A reporter complex.

\subsection{GLUCOSE METABOLISM AND CELL CYCLE PROGRESSION}

Sugar regulation is a complex process in plants. Multicellular organisms require long-distance and tissue-specific signaling mechanisms and coordination of developmental and environmental responses. As photosynthetic organisms, plants are made up of sugar exporting (source) and sugar importing (sink) tissues and organs (Rolland et al., 2006). Sugar metabolism is a dynamic process, and the sessile nature of plants requires the ability of sugar storage and mobilization throughout development in response to environmental signals such as diurnal changes or biotic and abiotic stresses (Blasing et al., 2005; Borisjuk et al., 2003; Roitsch, 1999; Smith et al., 2005; Weber et al., 2005). Sucrose is the final product of photosynthesis and the major transport form of sugar in the plant 
cell. Sucrose is cleaved into hexoses by cell wall invertases or sucrose synthase (Schwebel-Dugue et al., 1994) upon its entry into the cell. Photosynthesis and carbon metabolism are subject to feedback regulation and are major targets in sugar signaling. It is known that low sugar conditions upregulate source activities such as photosynthesis, nutrient mobilization, and export, while sink activities such as growth and storage are upregulated by various carbon sources revealing that source and sink demands are rigorously coordinated through metabolic regulation and sugar-signaling mechanisms. The sucrose hydrolytic hexose products, glucose and fructose have been shown to have major effects on plant growth and metabolism in addition to sucrose (Rolland et al., 2006).

Photosynthates are generated in the Calvin cycle and exported as triosephosphates into the cytosol where they are used in glycolysis or converted to sucrose (Rolland et al., 2006). Hexose sugars (e.g. glucose, fructose) can be combined to form structural sugars such as cellulose and hemicellulose that can be integrated into the cell wall or into starch in the chloroplasts and plastids. The alternative pathway for these sugars is catabolism in which large molecules such as starch are broken down into smaller three carbon molecules (triose sugar pool). Components of the catabolic pathway (triose sugar pool) can be oxidized in the glycolytic pathway or supplied to the biosynthetic pathways to form lipids, nucleic acids or proteins (Buchanan et al., 2000). The catabolic process of glucose and fructose degradation into three carbon molecules is a reversible process. Sucrose can be regenerated at any point in the pathway in response to environmental signals to regulate the metabolism process of the plant. For 
example, excess photosynthate is stored as starch in the chloroplast as a result of when glucose production exceeds the metabolic demands of the cell during the day (Caspar et al., 1985) while starch is broken down from chloroplasts in leaf cells and plastids in starch-storing organs when the sugar level is low during the nights or periods of dark (Smith et al., 2005; Weber et al., 2005). Enzymes implicated in metabolism are regulated by complex regulatory systems with lots of negative feedback loops. Hexokinase $(\mathrm{HK})$ is considered to be the main sensing molecule of catabolite repression triggered by glucose (Ehness and Entian, 1980; Entian and Frhlich, 1984; Ma and Bostein, 1986; Ma et al., 1989; Rose et al., 1991). Jang and Sheen (1994) revealed that glucose and other hexoses are the direct signals triggering photosynthetic repression through phosphorylation by the intracellular sugar sensor, hexokinase (HXK).

Sugars (sucrose, glucose) have been shown to promote mitotic activity in a variety of plant tissues and organs. The plant cell cycle as in other eukaryotic organisms occurs in a firmly regulated mode. The cell cycle consists of five separate phases: G1 phase, $S$ phase (DNA synthesis), G2 phase (as a group known as interphase), and the $M$ phase (mitosis). The $M$ phase is composed of two tightly - coupled processes: mitosis, when the cell's chromosomes are divided between the two daughter cells and cytokinesis, in which the cell's cytoplasm divides into two distinct cells. G1, and G2 are the two gap phases of the cell cycle that separate the segregation of chromosomes (M phase, mitosis) and replication of DNA (S phase). The main control points of the cell cycle are at the $\mathrm{G} 1 / \mathrm{S}$ and G2/M transitions (Stals and Inze, 2001). Activation of each phase 
depends on the proper completion of the previous phase. Progression of the cell cycle is controlled by two classes of regulatory molecules, cyclins and cyclindependent kinases (CDKs). Cyclins are activated by phosphorylation through CDKs. Arabidopsis contains 32 cyclins that regulate cell cycle progression (Inze, 2005). The cyclin dependent kinases (CDKs) are regulated in numerous ways through the association with cyclins, Kip-related proteins (KRP), CDK subunits (CKS) and by activating (CAKs, CDK activating kinases) or inhibiting phosphorylation kinases (Mironov et al., 1999; Dewitte and Murray, 2003). Plants have different groups of cyclins: D-cyclins, A-cyclins, and B-cyclins. D-cyclins were found to play a role during the G1 phase (Rossi and Varotto, 2002; den Boer and Murray, 2000; Meijer and Murray, 2000), as well as for entrance into mitosis (Sorrel et al., 1999), whereas A-cyclins are particularly important at the G1/S transition (Yu et al., 2003) and during S phase (Reichheld et al., 1996), while B-cyclins play a role at the G2/M transition in releasing the cell into mitosis (Reichheld et al., 1996; Criqui et al., 2000).

Plant growth factors such as cytokinins, brassinosteroids, sucrose and gibberellins have been shown to modulate the expression of plant cyclins, especially D-cyclins (Stals and Inze, 2001). Further, Hartig and Beck (2005) have shown that glucose modulates the rate of cell cycle progression in tobacco cell tissue culture. Cells were able to proceed through the cycle when they had accumulated enough starch, without the application of exogenous sugars. It has been shown that the rate of the cell cycle progression appears to be correlated to endogenous sugar levels rather than to glucose uptake by the cell verifying that 
hexokinase signaling is involved in the detection of endogenous sugar level (Sheen et al., 1999). Further, the expression of cyclin proteins CYCD2, CYCD1, CYCD3, CYCD2 and CYCA3, CYCD3 correlate to the level of endogenous sugars. In addition, the length of each $G 1$, and $G 2$ phases may be lengthened or shortened by the application or removal of sugar (Hartig and Beck, 2005). This shows that sugars play key roles in coordinating the genes regulated to the cell cycle progression as well as have an impact on plant tissue and organ development.

\subsection{GLUCOSE AND PLANT DEVELOPMENT}

Various plant developmental, physiological and metabolic processes are regulated in response to alterations in the levels or flux of soluble sugars, such as glucose and sucrose (reviewed in Graham, 1996; Koch, 1996; Smeekens, 1998, 2000; Wobus and Weber, 1999; Yu, 1999; Gibson, 2000; Pego et al., 2000; Rolland et al., 2002; Rook and Bevan, 2003). Glucose is a critical molecule at the cellular and developmental levels. It is a key regulator of critical processes such as germination; seedling development; root, stem, and shoot growth; photosynthesis; carbon and nitrogen metabolism; flowering; stress responses; and senescence (Moore et al., 2003).

Plant sugar responses are complex and involve numerous response pathways. Characterization of transgenic plant lines over-expressing plant/yeast hexokinases and analysis of mutations in the plant HEXOKINASE1 gene have showed that hexokinases play a critical role in the glucose response (Jang et al., 
1997; Moore et al., 2003) and revealed the hexokinase-dependent sugar response pathway (Graham et al., 1994; Jang and Sheen, 1994).

The fact that hexokinases play important roles in glucose metabolism complicates the role of hexokinase in the plant glucose response. Despite the role of hexokinase in glucose response, identification of genes regulated by sucrose and not hexoses indicate that there are other factors beside hexokinase that function as sugar sensors (Chiou and Bush, 1998; Rook et al., 1998; and Barker et al., 2000). Moreover, the sugar-response pathway has been found to interact with many other plant response pathways including the phytohormone response pathways (Leaon and Sheen, 2003). Glucose mutant screens have generated many mutant genes that are involved in the glucose response pathway (Zhou et al., 1998; Laby et al., 2000; Gibson et al., 2001). These mutants show an altered glucose response and some of them also showed altered responses to phytochormones as well. Screens for phytohormone response mutants abscisic acid have identified loci also identified in mutant glucose-response screens such as GIN1/ABA2, GIN5/ABA3, and GIN6/ABI4 alleles (Zhou et al., 1998; Laby et al., 2000; Gibson et al., 2001) indicating that glucose determines in part how plants respond to hormones and serve as a signaling molecule during different stages of plants development. Table 5 shows sugar - response mutants defective in phytohormone responses.

The following examples illustrate the role of glucose in plant development. For example, glucose aids in regulating the transition from growth by cell division to growth by cell expansion and reserve accumulation in developing embryos 
(Wobus and Weber, 1999; Borisjuk et al., 2003). In addition, a study done on cotyledons of developing fava beans showed that the undifferentiated, mitotically active tissue contained high levels of glucose whereas the most differentiated tissue contained low levels of glucose (Borisjuk et al., 2003). Also, experiment with the formation of adventitious roots in dark grown plants in the presence of glucose, sucrose or fructose resulted in the induction of adventitious roots at sites where the hypocotyls remained in contact with the media indicating a local absorption of the carbohydrate and illustrating glucose role in plant development (Takahashi et al., 2003).

Glucose regulates early seedling development and serves as a signaling molecule. For example, Arabidopsis seedlings grown on $6 \%$ glucose $(330 \mathrm{mM})$ were unable to green, expand their cotyledons or develop true leaves (Zhou et al., 1998). This inhibitory effect of exogenous glucose has allowed for the isolation of glucose hypersensitive and resistant genes as mentioned earlier and revealed a role for glucose as a signaling molecule. For example, gin2 and gin1 mutants (glucose insensitive) were able to grow at high concentration (6\% glucose $(330 \mathrm{mM}))$ while wild-type plants development was arrested by glucose indicating that these genes altered glucose signaling and resulted in the alteration of plant development (Zhou et al., 1998). 
Table 5: Selected sugar and phytohormone defect responses

\begin{tabular}{|c|c|c|c|}
\hline Original mutant & Original selection & $\begin{array}{l}\text { Mutant } \\
\text { Phenotype }\end{array}$ & lic mutant \\
\hline \multirow[t]{2}{*}{ gin } & $\begin{array}{l}\text { Reduced sensitivity to } \\
\text { inhibition of early seedling } \\
\text { development by glucose }\end{array}$ & $\begin{array}{l}\text { gin } 1 \text { - defective in } \\
\text { abscisic acid biosynthesis }\end{array}$ & aba2 \\
\hline & & $\begin{array}{l}\text { gin2 - auxin insensitive, } \\
\text { cytokinin hypersensitive } \\
\text { gin4 - ethylene constitutive } \\
\text { response } \\
\text { gin5 - defective in abscisic acid } \\
\text { biosynthesis } \\
\text { gin6 - abscisic acid-insensitive }\end{array}$ & $\begin{array}{l}\text { hxk1 } \\
\text { ctr1 } \\
\text { aba3 } \\
\text { abi4 }\end{array}$ \\
\hline \multirow[t]{2}{*}{ isi } & $\begin{array}{l}\text { Impaired sugar-inducible } \\
\text { expression of ADP-glucose } \\
\text { oyrophosphorylase subunit } \\
\text { ApL3 }\end{array}$ & isi3 - abscisic acid-insensitive & \\
\hline & & $\begin{array}{l}\text { isi } 4 \text { - defective in abscisic acid } \\
\text { biosynthesis }\end{array}$ & aba2 \\
\hline prl & $\begin{array}{l}\text { ncreased sensitivity to } \\
\text { inhibition of early seedling } \\
\text { development by sugars }\end{array}$ & $\begin{array}{l}\text { prl1 - abscisic acid, auxin, cytokinin, } \\
\text { and ethylene-hypersensitive }\end{array}$ & prl1 \\
\hline \multirow[t]{2}{*}{ sis } & $\begin{array}{l}\text { Reduced sensitivity to } \\
\text { hibition of early seedling } \\
\text { evelopment by glucose or } \\
\text { ucrose }\end{array}$ & sis 1 - ethylene constitutive response & ctr1 \\
\hline & & $\begin{array}{l}\text { sis } 4 \text { - defective in abscisic acid } \\
\text { biosynthesis } \\
\text { sis } 5 \text { - abscisic acid insensitive }\end{array}$ & $\begin{array}{l}a b a 2 \\
a b i 4\end{array}$ \\
\hline sun & $\begin{array}{l}\text { Reduced sensitivity to } \\
\text { sugar repression of } \\
\text { plastocyanin } \\
\text { expression }\end{array}$ & sun6 - abscisic acid-insensitive & abi4 \\
\hline
\end{tabular}




\subsection{GLUCOSE AND CHROMATIN REMODELING}

The relationship between glucose signaling and chromatin remodeling has not been studied extensively. Nicolai et al., (2006) and Blasing et al., (2005) have linked glucose to chromatin remodeling mechanisms. Nicolai et al., 2006 investigated the effect of sucrose starvation on the transcriptome of $A$. thaliana cell suspension cultures. Sucrose starvation resulted in the repression of the histone deacetylase AtHD1 (At4g38130) and simultaneous increase in $\mathrm{H} 4$ acetylation that was measured by the immunoprecipitation of chromatin with an H4-acetylation binding antibody. This indicates that the state of histone protein modification is a function of the metabolic state of the cell. Moreover, analysis of the pgm mutant showed two histone deacetylases that show greater than two fold increases in transcript abundance during a diurnal cycle. In addition, other genes implicated in DNA, RNA, and protein synthesis were also induced during periods of high glucose (light) and repressed during periods of low glucose (dark) conditions (Blasing et al., 2005).

Analysis of the Saccharomyces cerevisiae ADH2 promoter region in its chromosomal location under repressive (high-glucose) conditions and during derepression resulted in the finding that nucleosomes the ADH2 promoter of the gene remodelled. The main ones were positioned at the RNA initiation site (nucleosome +1), at the TATA box (nucleosomes -1), and upstream of the ADR1binding site (USA1) (nucleosome -2) under glucose (repressed conditions). The USA1 sequence and the adjacent USA2 sequence comprised a nucleosome-free region. Nucleosomes -1 and +1 were destabilized after glucose depletion and 
had become so before the appearance of ADH2 mRNA. In addition, under high rates of transcription, nucleosomes -2 and +2 also went through rearrangement. Moreover, detection of this chromatin remodeling in spheroplasts prepared from cells grown in minimal medium required the addition of a minimum amount of glucose. However, cells which lack the ADR1 protein did not undergo any of these chromatin modifications upon glucose depletion (Verdone et al., 1996).

\subsection{GLUCOSE SENSING IN PLANTS}

Sugars are an important nutrient that can effect plant growth and development. Sugars can act as regulatory signals that control the expression of various genes involved in numerous processes in the plant life cycle (Koch, 1996; Jang and Sheen, 1997; Smeekens, 1998; Lalonde et al., 1999; Roitsch, 1999; Sheen et al., 1999). Sugar sensing and signaling in plants is a complex phenomenon due to the interaction between source and sink tissues. Experimental evidence suggests the existence of three different glucose sensing systems in plant cells (Figure 1). The first is a hexokinase (HXK)-dependent pathway in which gene expression is mediated through the AtHXK1 signaling function (Jang et al., 1997; Xiao et al., 2000). The second is a glycolysisdependent pathway that depends on the catalytic activity of HXK (Xiao et al., 2000). The third is a HXK-independent pathway (Martin et al.,1997; Mita et al., 1997; Roitsch, 1999; Xiao et al., 2000; Ciereszko et al., 2001). 
Identification of the HXK-dependent pathway was demonstrated by using plants mutant in the HXK-signaling function (Moore et al., 2003), transgenic plants with a modified expression of the AtHXK1 gene (Jang et al., 1997), by using mannoheptulose, a specific inhibitor of HXK, or by using glucose analogues such as 2-deoxyglucose (Pego et al., 1997; Graham et al., 1994 and Prata et al., 1997). Glucose-phosphorylating activity is not critical for HXK signaling since overexpression of a heterologous yeast $Y H X K 2$ that can phosphorylate glucose in the cells but can not provide the signaling function did not cause any effect on the expression of photosynthetic genes (Xiao et al., 2000) Mutations in the HXK1 gene (gin2) lead to phenotypic abnormalities in numerous process associated with sugar signaling including gene expression, cell proliferation, root and inflorescence growth, leaf expansion and senescence. Since the gin2 phenotype was rescued by expression of HXK1 deficient in catalytic activity, HXK1 was proposed to function as a sugar sensor independent of its role in glucose metabolism (Moore et al., 2003). Moreover, transgenic plants overexpressing HXK showed sugar hypersensitivity whereas antisense expression caused sugar hyposensitivity (Jang and Sheen, 1997).

The HXK - glycolysis-dependent pathway depends on the catalytic activity of HXK. Activation of genes in this system depends on one or more enzymes or intermediates of glycolysis downstream of hexokinase. Glucose signaling triggered by metabolites downstream of the sugar phosphates in glycolysis has been shown to induce the expression of pathogenesis related (PR) genes 
(Sheen et al., 1999). However, the direct metabolic signals and sensor for the regulation of $P R$ genes by glucose is still unknown.

Expression of numerous plants genes such as Chenopodium genes encoding extracellular invertase and sucrose synthase (Roitsch et al., 1995) and Arabidopsis thaliana genes encoding patatin storage protein (Martin et al., 1997; Ehness et al., 1997) are induced by glucose analogues such as 6-deoxyglucose and 3-O-methylglucose that cannot be phosphorylated, suggesting the existence of a HXK-independent pathway. The existence of two glucose transporter-like proteins, Rgt2 and Snf3, that act as sugar sensors in yeast suggested the possibility of a similar mechanism existing in plants (Lalonde et al., 1999; Roitsch, 1999; Sheen et al., 1999) as shown in Figure 2. Rgt2 and Snf3 resemble typical cell surface receptors that can transduce the glucose signal to a HXKindependent pathway rather than transport glucose (Johnston, 1999). There have been 26 homologous sequences of monosaccharide transporters identified in Arabidopsis thaliana. Two of the 26 homologues appear to encode transporters with extended intracellular loops (Lalonde et al., 1999) that might play a role similar to the Snf3 and Rgt2 yeast proteins in transmitting the glucose signal to a HXK-independent pathway (Rolland et al., 2001). Three glucose sensing system other than the Hxt system have been characterized in yeast (Figure 2). Each system perceives the glucose signal and transmits it differently. The first system is through the Snf1 protein kinase which causes gene repression at a high levelddxs of glucose. The second system operates through the Snf3 and Rgt2 glucose sensors to induce expression of genes encoding glucose transporters. 
The third system use the Gpr1 G-protein coupled receptor with cyclic AMP as the second messenger (Rolland et al., 2002). It has been speculated that similar systems might exist in plants that transmit the glucose signal to a HXKindependent pathway.

Glucose plays a role in many aspects of plant growth and development such as germination, seedling development, photosynthesis, carbon and nitrogen metabolism, flowering, stress responses, and senescence (Rolland et al., 2002, 2006; Yoshida, 2003; Gibson, 2005; Wingler et al., 2006). Glucose effects on photosynthetic genes and seedling development have been shown to be mediated through the AtHXK1- dependent signaling pathway (Xiao et al., 2000). On the other hand, glucose inhibition of germination was shown to occur by AtHXK1 and ABA/AB/4 independent-signaling pathway (Price et al., 2003). Thus, in the germination process glucose signaling seems to be sensed and transmitted by a different glucose sensor other than the AtHXK1. Chen et al., (2006) showed that the regulator of G-protein signaling (RGS) protein is involved in sugar and ABA signaling in Arabidopsis thaliana seed germination. Johnston et al., (2007) also demonstrated that the heterotrimeric $\mathrm{G}$ protein signaling is important for cell-proliferative and glucose-sensing signal transduction pathways in Arabidopsis thaliana. AtRGS1 has a predicted seven-transmembrane structure (similar to a GPCR) and an RGS box with GTPase-accelerating activity that desensitizes the G-protein-mediated signaling (Figure 3). Extracellular signal molecules interact with G-protein-coupled receptors (GPCRs), to generate conformation changes in GPCR structure and as a result initiates intracellular 
signaling by disassociating the G-protein $\alpha$-subunit from the $\beta-$-subunit complex and facilitating the exchange of GTP for GDP on the G-protein $\alpha$-subunit. The activated GTP-bound $\alpha$-subunit and $\beta \sqsupset$-subunits then interact separately with a variety of down-stream effectors (Figure 3). The period of time in which Gasubunit remains in an active GTP-bound form is limited since the Ga-subunit itself possesses an intrinsic GTPase activity. The signaling terminates once the Ga-subunit hydrolyzes GTP to GDP and the heterotrimer reforms (Gudermann et al., 1997; Ford et al., 1998; Li et al., 1998; Wall et al., 1998). Hence, heterotrimeric G-proteins act as molecular switches that coordinate the transfer of information from environmental signals to the cell interior (Chen et al., 2006). In another study by Chen and Jones (2004) in which they used different kinds of sugars and their analogs were used to study the effect on seedling development. they found that Atrgs 1 mutants was less sensitive to high concentration of glucose indicating that AtRGS1 may be involved in the regulation of seedling development responses to sugar signaling. Moreover, AtRGS1 is speculated to function in a glucose-HXK-independent signaling pathway since sugar metabolism and phosphorylation by HXK are not required for AtRGS1-mediated signaling (Chen and Jones, 2004). 


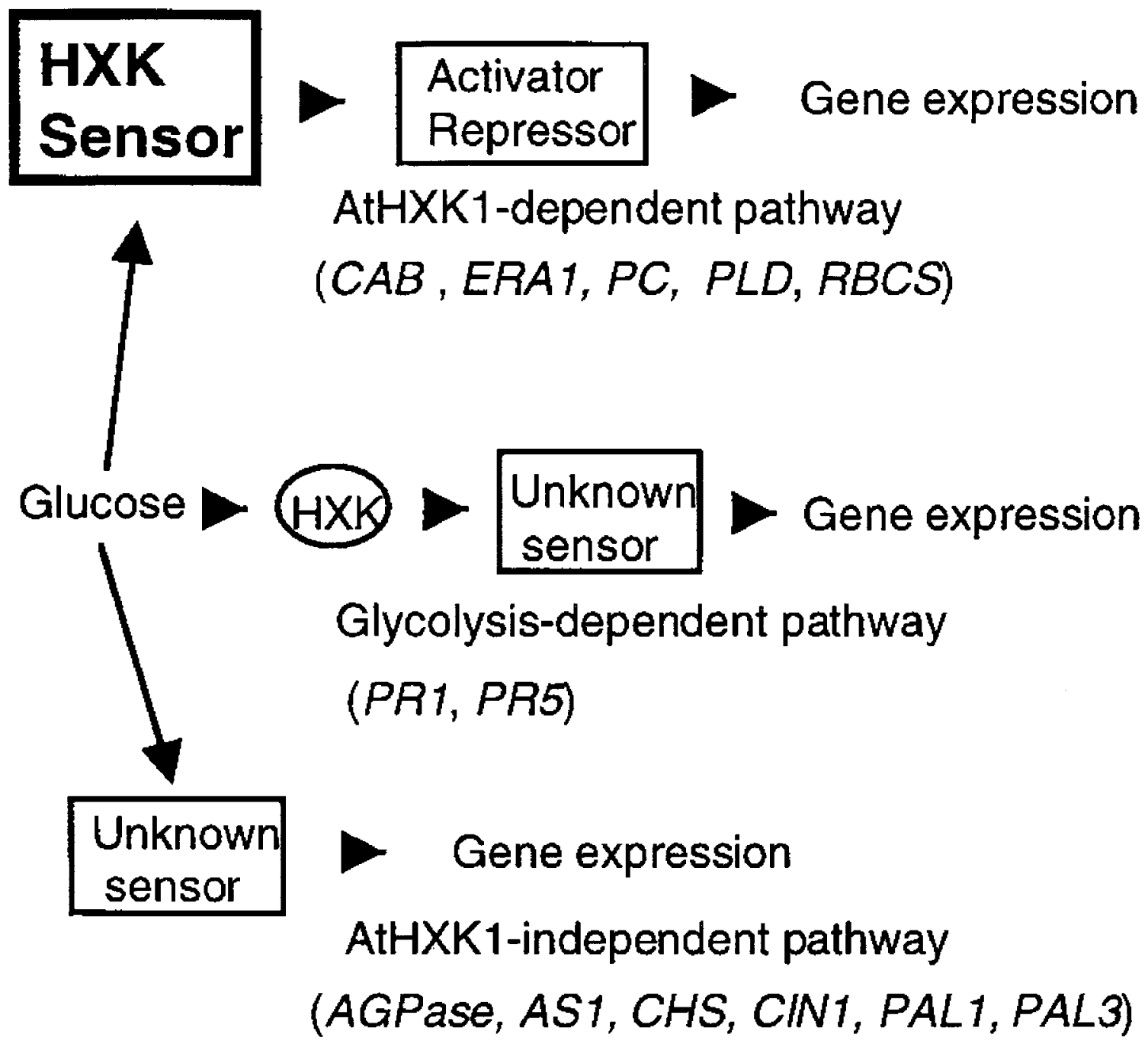

Figure 1: Three glucose sensing and signal transduction pathway are proposed to operate in plant cells (Xiao et al., 2000). The expression of the genes in parentheses are believed to be targets of the indicated pathway. 


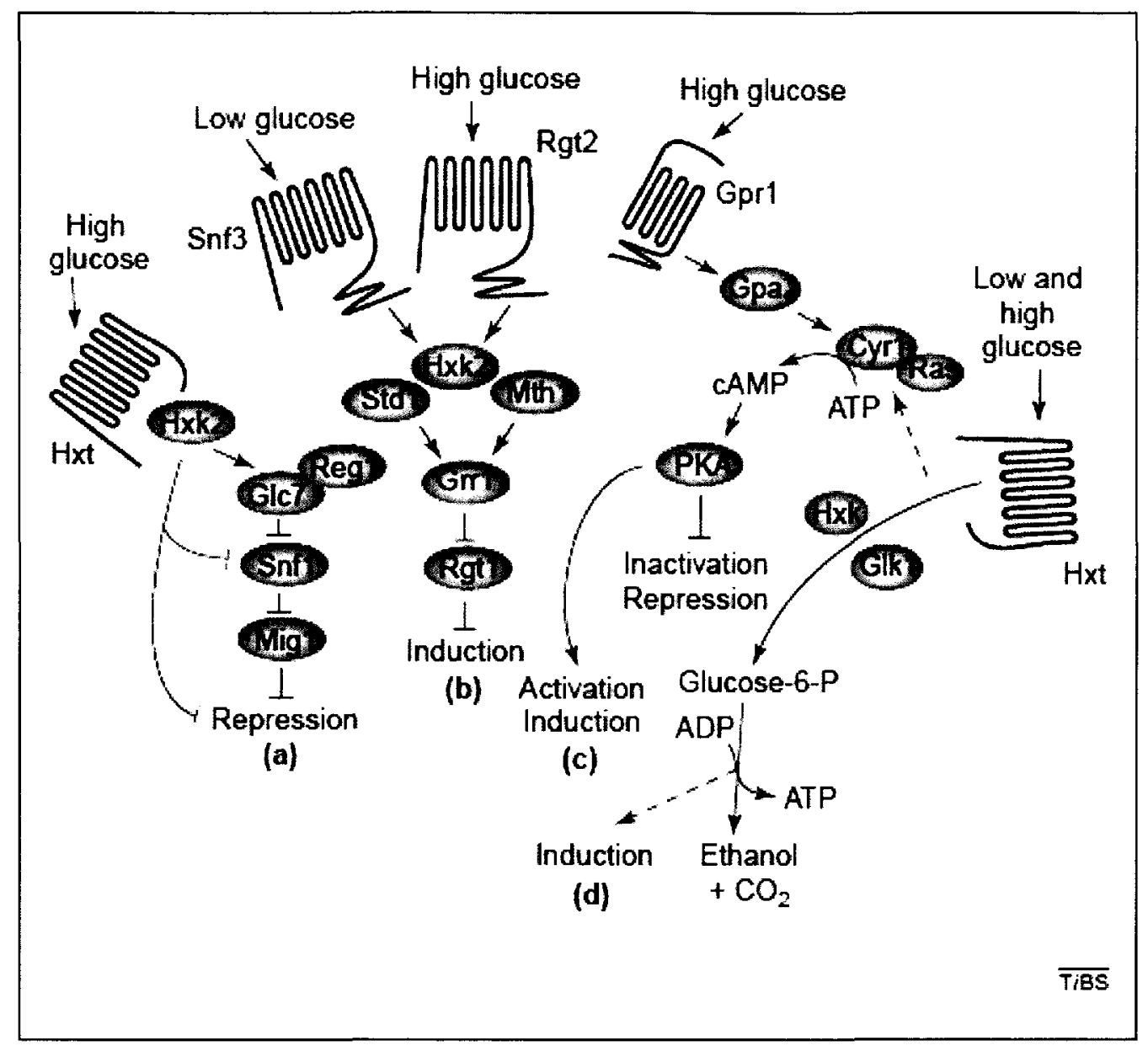

Figure 2: Glucose sensing in yeast. Four distinct glucose signaling systems are demonstrated. (a) The major glucose or catabolite repression pathway which is activated by high glucose and causes repression of genes involved in respiration, gluconeogenesis and the metabolism of alternative sugars. (b) The second glucose signaling pathway controls the induction of hexose carrier genes. Two transporter homologues Snf3 and Rgt2 are believed to control the induction of specific glucose carrier genes by low and high glucose levels respectively. (c) Glucose activation of cAMP synthesis entails two inputs: glucose phosphorylation either by glucokinase 1 (Glk1) or by one of the two hexokinases (Hxk1 and Hxk2) in which only a low level of glucose is required, and activation of the G-proteincoupled receptor (GPCR) Gpr1, which requires a high level of glucose. (d) The fourth pathway is responsible for induction of glycolytic genes by glucose (Rolland et al., 2001). 


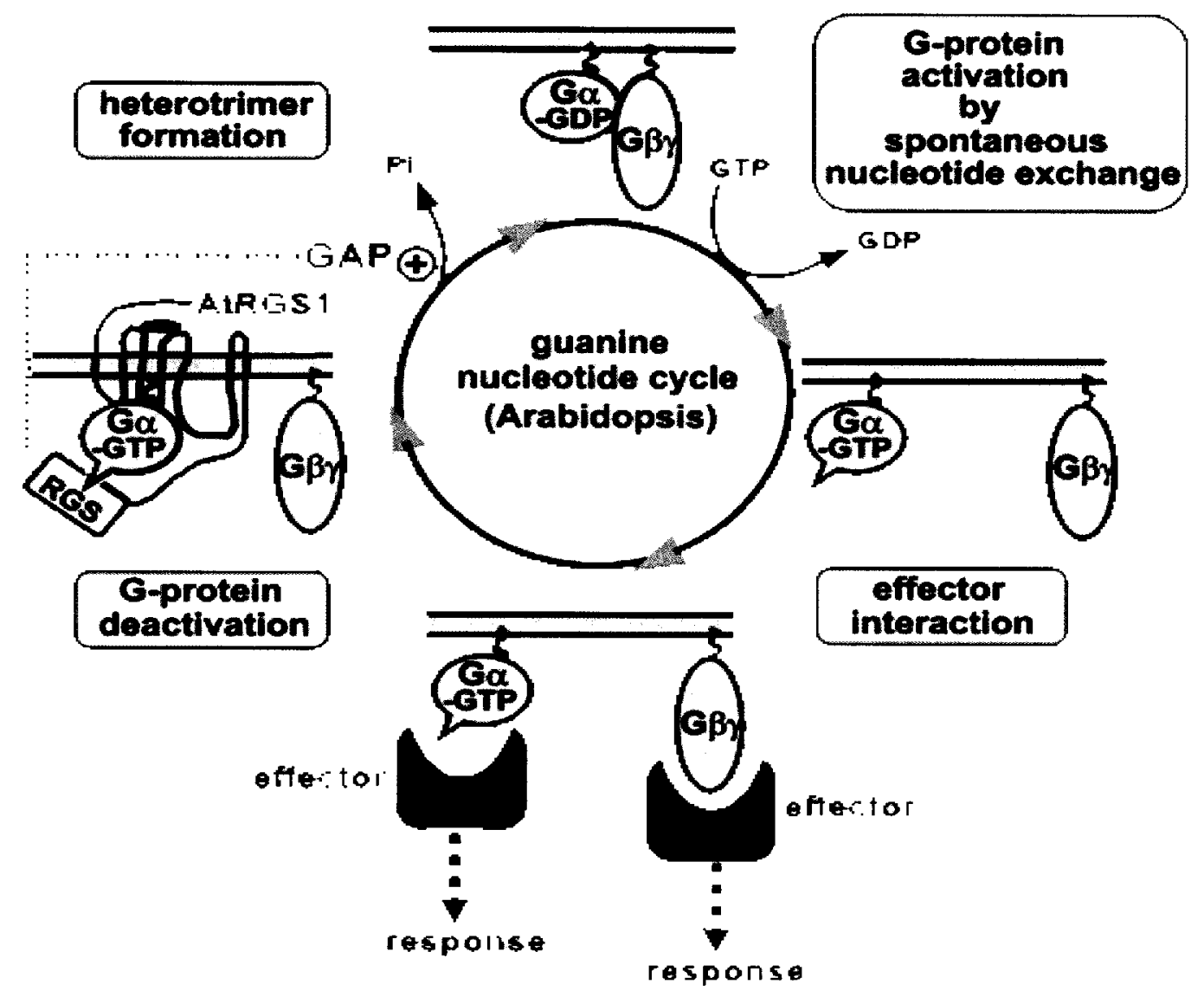

Figure 3: Heterotrimeric G-protein modes of action. Guanine nucleotide cycle of heterotrimeric $G \alpha$ in Arabidopsis. Arabidopsis genome encodes one

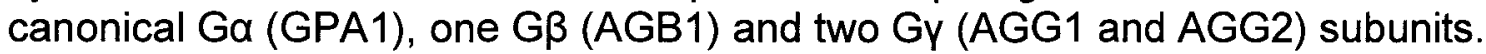
GPA1 is an unusual heterotrimeric $\mathrm{Ga}$ because of a rapid nucleotide exchange and the slowest GTPase activity. Thus, the GTP hydrolysis, rather than GDP release, is likely the rate-limiting step in the guanine nucleotide cycle of GPA1. Classical GPCRs (possessing GEF activity on $\mathrm{Ga}$ ) may not be required for the activation of G-protein signaling because GPA1 is in the activated state (GTP-bound) by default. Instead, AtRGS1, the sole RGS protein in Arabidopsis, functions as a critical regulator of G-protein signaling by accelerating the slow, intrinsic GTPase activity of GPA1. It is likely that AtRGS1 acts as a ligand-regulated 7TM GAP for GPA1. The exact ligand for AtRGS1 that modulates GAP activity has not been identified, though D-glucose has been shown to stimulate the interaction between AtRGS1 and GPA1. Upon activation, $G \alpha$ and $G \beta y$ subunits are shown to dissociate in this model, but Arabidopsis G-proteins may signal through nondissociable heterotrimers (Chen, 2008). 


\subsection{GLUCOSE AND THE PLANT HORMONE ABA}

Glucose and other sugar molecules have been shown to be primary signal molecules that affect seed germination and early seedling development (Gibson 2000, Dekkers et al., 2004, Gibson 2005). Studies have demonstrated that the effects of sugars on seed germination are complex and can be associated with ABA biosynthesis and signaling (Gibson 2004, Yuan and Wysocka-Diller 2006, Rook et al., 2006, Finkelstein et al., 2008). ChIP studies have verified that the expression of various genes associated with carbohydrate metabolism, signal transduction, and metabolite transport are co-regulated by sugar and ABA (Price et al., 2004, Li et al., 2006). For example, genes involved in ABA biosynthesis such as $A B A 2$ and NCED3 are induced by glucose in responses in Arabidopsis (Cheng et al., 2002, Chen et al., 2006). Furthermore, the ABA-deficient mutants, aba2/gin1 and aba3/gin5, and ABA-insensitive mutants, abi4/gin6, abi5 and abi8, are also insensitive to sugar (Gibson 2005, Dekkers et al., 2008) indicating that the ABA biosynthesis and signaling pathways are directly linked to glucose signaling pathways.

In addition, several experiments have revealed that the endogenous level of $\mathrm{ABA}$ is regulated by both its biosynthesis and catabolism through the 8hydroxylase (cytochrome P450 monooxygenase) that converts $A B A$ to phaseic acid (PA) (the primary catabolites of ABA) (Kushiro et al., 2004, Saito et al., 2004). A number of experiments have shown that the ABA 8-hydroxylase family

plays a major role in the regulation of endogenous $A B A$ levels during seed 
development and germination in Arabidopsis (Millar et al., 2006, Okamoto et al., 2006).

Various studies have demonstrated that germination is delayed in the presence of glucose, in part due to upregulation of the ABA biosynthesis genes, $A B A 2$ and NCED3, through glucose signaling (Cheng et al., 2002, Chen et al., 2006). A recent study in rice on the effect of glucose on ABA catabolism genes in revealed that the expression of ABA 8 -hydroxylase (OsABA8ox2 and OsABA8ox3) is significantly suppressed by glucose signaling which in turn results in the delay of germination, again indicating that $A B A$ catabolism are responsible for the increase in $A B A$ level at high glucose together with changes in the ABA biosynthesis genes (Zhu et al., 2009).

\subsection{ABSCISIC ACID AND EPIGENTIC PROCESES IN PLANT DEVELOPMENT AND STRESS RESPONSES}

The phytohormone abscisic acid (ABA) regulates aspects of plant growth and development including germination, lateral root development, seedling growth, seed development, seed dormancy, transition from vegetative to reproductive phase as well as the responses environmental stresses such as drought, cold, and salt (Finkelstein et al., 2002). There exist multiple ABA receptors and ABA signal transduction pathways (Shen et al., 2006; Liu et al., 2007). It is believed that $A B A$ responses are regulated by $A B A$-mediated transcriptional regulation (reviewed in Schroeder et al., 2001). Latest discoveries

show that besides genetic regulation, epigenetic regulation plays a key role in 
ABA-mediated plant processes as well and reveal a potential link between $A B A$ signaling and chromatin remodeling. The following are examples of ABAmediated epigenetic processes in seeds.

Plants have adaptive mechanisms in seeds to prevent germination in osmotic stress conditions during the initial phase of germination. For example exogenous application of ABA or osmotic stress within 48 hours of imbibition results in the delay of Arabidopsis seed germination. Germination arrest is arbitrated by the induction and prolonged expression of $A B / 3$ and $A B / 5$ transcription factors. Chromatin remodeling is essential for $A B A$ and osmotic stress induction of germination arrest. Expression of the Arabidopsis chromatin remodeling factor PKL (SWI/SNF) is induced by imbibition and mediates repression of embryonic traits during germination (Henderson et al., 2004; Li et al., 2005). During germination LEAFY COTYLEDON1 (LEC1, a transcriptional regulation that promotes embryonic identity) and FUSCA3 (FUS3) are repressed in wild type, but expressed in pkl mutants upon seed imbibition (Ogas et al., 1999). It has been shown that the $p k l$ mutants display a high expression of $A B / 3$ and $A B / 5$ and a hypersensitive germination response to exogenous $A B A$. Microarray study comparing wild-type and $p k l$ mutant seeds treated with or without $A B A$ showed that ABA-treated $p k l$ mutant seeds had 2.0-2.5 fold lower $\mathrm{H} 3-\mathrm{K} 9$ and $\mathrm{H} 3-\mathrm{K} 27$ methylation levels at $A B / 3$ and $A B / 5$ promoters in comparison to wild-type. Thus, PKL effects on chromatin structure by repressing $A B / 3$ and $A B / 5$ and a few other late embryogenesis abundant genes that are expressed after imbibition to promote germination (Perruc et al., 2007). 
Further, histone deacetylation is also involved in ABA sensitivity during germination. A number of the histone deacetylase (HDACs) members are repressed by ABA in Arabidopsis (Sridha and Wu 2006) and rice (Fu et al., 2007). Down-regulation Arabidopsis APETALA2/EREBP (AtERF7) transcription factor expression in HDA19 mutants revealed a role for HDA19 in ABA sensitivity during seed germination. AtERF7 interacts with AtSin3 (a global corepressor of transcription) which then interacts with HDA19. HDA19 and AtSin3 enhance the AtERF7 mediated transcriptional repression. Down regulation of AtERF7 and AtSin3 function by RNA interference (RNAi) resulted in the enhanced of $A B A$ sensitivity during germination and seedling growth. Hence, ABA signaling seems to repress the genes through histone acetylation (Song et al., 2005). In addition, a repression of HDA6 function via RNAi treated with trichostatin A (HDAC inhibitor) resulted in growth arrest and elevated expression of LEC1, FUS3, and $A B / 3$ during germination, while the growth arrest phenotype of the HDA6-RNAi repression plants was suppressed in lec1, fus3, and abi3 mutants. Moreover, double repression of $H D A 6 / H D A 19$ function showed growth arrest after germination and the formation of embryo-like structures concluding that HDA6 and HDA19 redundantly regulate the repression of embryonic properties and growth arrest during germination (Tanaka et al., 2008). It has been also shown that Arabidopsis HDA6 is implicated in transgene silencing and the regulation of ribosomal RNA transcription (Probst et al., 2004; Earley et al., 2006). Thus, it appears that histone deacetylation plays an essential role in the induction of embryonic genes and germination inhibition. 
Expression analysis of rice HDAC genes demonstrated that the expression of numerous $H D A C s$ is not only influenced by abiotic stresses but by plant hormones as well. In rice, it has been shown that $A B A$ represses the expression of HDT701, HDT702, SRT701 and SRT702 (Fu et al., 2007). Abiotic stresses result in the induction of $A B A$ accumulation which in turn might cause HDACs - mediated changes in gene expression. In Arabidopsis, HD2C gene expression was repressed in wild-type upon $A B A$ treatment, whereas transgenic Arabidopsis plants overexpressing AtHD2C displayed an ABA-insensitive phenotype as well as higher tolerance to salt and drought stresses (Sirdha and Wu 2006). The increased tolerance of transgenic plants overexpressing AtHD2C was partially due to an increase of ABA-reponsive LEA- like gene expression (RD29B and RAB18), and a decrease in $A B / 2$ and $A D H 1$ (alcohol dehydrogenase 1), SKOR (K+ outward rectifier) KAT1 and KAT2 (K+ inward rectifier) expression (Sirdha and $\mathrm{Wu}, 2006$ ). Hence, these results suggest that ABA mediates abiotic stress responses via the regulation of histone acetylation levels.

\subsection{THESIS OBJECTIVES}

The purpose of this study is to investigate the functional importance of the HD2 plant-specific class of histone deacetylases during seedling germination and development. To elucidate a role for HD2 family members, null mutants were analyzed to identify defects in germination and developmental abnormalities. An earlier microarray study (Colville, 2007) showed a link between soluble 
carbohydrates (glucose, sucrose, fructose) and HD2 expression which was further validated by semi-quantitative reverse transcription PCR (RT-PCR). My objective was to quantify the induction of the various $H D 2$ members upon soluble carbohydrate treatment and to investigate the specificity of $H D 2$ induction using quantitative qPCR method. The roles of HD2 family members in the sugar pathway and its regulation by the plant stress hormone ABA were examined as a part of this research. Since, plants use HXK as a glucose sensor to interconnect nutrient, light, and hormone (such as $\mathrm{ABA}$ ) signaling networks for controlling growth and development in response to environmental changes (Moore et al., 2003) a AtHXK1/gin2 loss-of-function mutant line was employed to investigate the mechanism of glucose-mediated HD2 induction and determine whether the HXK-dependent glucose signal transduction pathway is involved in the regulation of $H D 2$ expression. In addition, a promoter analysis was used to identify sugar responsive elements within the promoter sequence of the HD2 genes. A promoter GUS fusion assay will be carried to test whether the induction is directly linked to the HD2 promoters. Furthermore, phenotypical analysis will be performed on single and double mutants of $A t H D 2 A$ and $A t H D 2 C$ members of the HD2 family as well as on over-expressed line of HD2A-GFP under different concentration of glucose as well as under osmotic stress conditions to identify any developmental abnormalities that might yield insight into the role of HD2 during plant development. Moreover the hypothesis of functional redundancy of the HD2 genes will be also investigated in this study. 


\section{CHAPTER 2}

\section{MATERIALS AND METHODS}

\subsection{Plant Materials and Growing Conditions}

Two ecotypes of wild-type Arabidopsis thaliana were used in this study, Columbia-O (Col-0) and Landsberg erecta (Ler). Publicly available collections of Arabidopsis thaliana mutant plants were searched for the HD2B and HD2D TDNA mutants and all available T-DNA insertion lines were obtained from the Arabidopsis Biological Resource Center (ABRC, Columbus, OH, USA) and Nottingham Arabidopsis Stock Centre (NASC, University of Nottingham, United Kingdom).

Plants were grown under various conditions. For the phenotypic analysis and genomic DNA and total RNA extraction, plants were grown in a controlledenvironment growth chamber (Percival scientific) under continuous light conditions (24 hours light) at a temperature of $22^{\circ} \mathrm{C}$. Plants required for other experiments such as seed amplification and seed harvesting of homozygous plants were transferred into soil and grown in a moderate-day photoperiod chamber ( 16 hour light/ 8 hour dark) at a temperature of $22^{\circ} \mathrm{C}$. For the induction of $H D 2$ gene expression by soluble carbohydrates, seeds were germinated under continuous light (24 hours light) for 24 hours at a temperature of $22^{\circ} \mathrm{C}$, followed by dark for 4 days.

Seeds were surface-sterilized with a solution of $70 \%$ ethanol for 2 minutes and then soaked in sterilizing solution ( $30 \%$ sodium hypochlorite (CLOROX $® \mathrm{v} / \mathrm{v}$ ) (The Clorox Company, CA, USA) $0.01 \%$ Triton-X (v/v) (Fischer Scientific, NH, 
USA)) for 8 minutes. They were rinsed four times with sterile water and stratified in water in the dark for 48 hours at $4^{\circ}$ Celsius to break dormancy before germinating on $1 / 2$ Murashige and SKoog Basal Salt Mixture (MS) (Sigma-Aldrich, UK) (w/v), 3\% sucrose (w/v), 0.7\% Phytagar (Sigma-Aldrich, UK) (w/v), pH 5.7 plates or soil.

2.2 Identification and Molecular Characterization of Homozygous T-DNA HD2B and $H D 2 D$ Insertion Lines

\subsubsection{Mutants and Transgenic Plants}

Publicly available collections of Arabidopsis thaliana mutant plants were searched for the $H D 2 B$ and $H D 2 D$ T-DNA mutants and all available T-DNA insertion lines were obtained (Table 6). Plants were grown under normal growth conditions in continuous light at $22^{\circ}$ Celsius. Leaf disks were collected from the ten days old seedlings into $1.5 \mathrm{ml}$ microcentrifuge tube and frozen at $-80^{\circ}$ Celsius for genotyping. Plants were transferred to soil and grown in 8 hour light/16 hour dark cycle. Mature seeds were harvested. 
Table 6: $H D 2 D$ and $H D 2 B$ mutant lines analyzed for phenotypic abnormalities

\begin{tabular}{|c|c|c|c|c|c|c|}
\hline GENE & Stock \# & Ecotype & $\begin{array}{c}\text { Selectable } \\
\text { Marker }^{2}\end{array}$ & Description $^{3}$ & Location & Source \\
\hline \multirow{7}{*}{$\begin{array}{l}H D 2 D \\
\text { (AT2G2 } \\
7840)\end{array}$} & SALK_104071 & Columbia-0 & Kanamycin & $\begin{array}{l}\text { T-DNA } \\
\text { insertion }\end{array}$ & $\begin{array}{l}1000- \\
\text { Promoter }\end{array}$ & ABRC \\
\hline & SALK_095273 & Columbia-0 & Kanamycin & $\begin{array}{l}\text { T-DNA } \\
\text { insertion }\end{array}$ & $\begin{array}{l}1000 \\
\text { Promoter }\end{array}$ & $A B R C$ \\
\hline & GT_5_10004 & Landsberg & Kanamycin & $\begin{array}{l}\text { Transposon } \\
\text { insertion }\end{array}$ & $\begin{array}{l}1000 \\
\text { Promoter }\end{array}$ & ABRC \\
\hline & 100010 & Landsberg & Kanamycin & $\begin{array}{l}\text { Transposon } \\
\text { insertion }\end{array}$ & $\begin{array}{l}1000 \\
\text { Promoter }\end{array}$ & $A B R C$ \\
\hline & GABI_278D04 & Columbia-0 & Sulfadiazine & $\begin{array}{l}\text { T-DNA } \\
\text { insertion }\end{array}$ & Intron & NASC \\
\hline & GABI_379G06 & Columbia-0 & Sulfadiazine & $\begin{array}{l}\text { T-DNA } \\
\text { insertion }\end{array}$ & Intron & NASC \\
\hline & GABI_836B08 & Columbia-0 & Sulfadiazine & $\begin{array}{l}\text { T-DNA } \\
\text { insertion }\end{array}$ & $\begin{array}{l}300- \\
\text { UTR5 }\end{array}$ & NASC \\
\hline \multirow{3}{*}{$\begin{array}{l}H D 2 B \\
\text { (AT5G2 } \\
2650)\end{array}$} & SALK_049380 & Columbia-0 & Kanamycin & $\begin{array}{l}\text { T-DNA } \\
\text { insertion }\end{array}$ & $\begin{array}{l}1000- \\
\text { Promoter }\end{array}$ & ABRC \\
\hline & SALK_113500 & Columbia-0 & Kanamycin & $\begin{array}{l}\text { T-DNA } \\
\text { insertion }\end{array}$ & $\begin{array}{l}\text { 1000- } \\
\text { Promoter }\end{array}$ & $\mathrm{ABRC}$ \\
\hline & $\begin{array}{l}\text { SAIK_1247_A } \\
02\end{array}$ & Columbia-0 & BASTA & $\begin{array}{l}\text { T-DNA } \\
\text { insertion }\end{array}$ & Intron & ABRC \\
\hline
\end{tabular}

$1 \quad$ Indicates the stock number of the mutant line from either The Arabidopsis Biological Resource Center (ABRC) or Nottingham Arabidopsis Stock Centre (NASC)

${ }_{2}$ Indicates the transgene carried within the mutant line for selection of genetic material inserted into the genome

${ }_{3}$ Indicates the type of mutant used in this analysis. Mutants were obtained from ABRC and NASC. T-DNA mutants were generated by Agrobacterium sp. Infiltration with a strain that carries the Ti plasmid. Part of the Ti Plasmid gets transformed into the plant host cell. This DNA integrates into the host cell genome and could disrupt the expression of gene in the immediate vicinity of the insertion. Transposon insertion mutants were obtained from ABRC. These mutants were transformed with an active transposable element which may inactivate the target gene if the insertion position was within the target gene. 


\subsubsection{Plant Genomic DNA Extraction and Genotyping}

Arabidopsis thaliana ecotype Columbia (Col-0) and Landsberg erecta (Ler) were used as the wild type control in this study. DNA extraction was carried out on all T-DNA insertion lines listed in Table 6 using the Extract-N-Amp ${ }^{\mathrm{TM}}$ Plant PCR Kit (Sigma-Aldrich, UK). Genomic DNA was extracted from leaf discs 0.5$0.7 \mathrm{~cm}$ diameter. Tissue was incubated in $100 \mu \mathrm{l}$ Extraction Solution at $95^{\circ} \mathrm{C}$ for 10 minutes. An equal volume of the Dilution Solution was then added to the extract to neutralize inhibitory substances prior to PCR. A portion of the DNA extract $(2 \mu l)$ was added directly to the optimized PCR mix supplied with gene specific primers for genotyping.

Plants homozygous for the T-DNA insertion in the $H D 2 B$ and $H D 2 D$ alleles were identified using PCR with primers which flank the T-DNA insertion for each stock line (Table 7). Primers were designed by the online tool of the Salk Institute Genomic Analysis Laboratory (http://signal.salk.edu/tdnaprimers.2.html). 
Table 7: Genotyping primers for the $H D 2 D$ and $H D 2 B$ T-DNA insertion lines

\begin{tabular}{|c|c|c|c|c|c|}
\hline Gene & \multirow[t]{2}{*}{ Stock \# } & \multicolumn{4}{|c|}{ Detection of Homozygous lines Using LP and RP Primers } \\
\hline \multirow{4}{*}{ HD2B } & & \multicolumn{2}{|c|}{\begin{tabular}{l|l} 
Primer & Left Primer (LP) \\
Name
\end{tabular}} & Right Primer (RP) & \multirow{2}{*}{$\begin{array}{l}\text { Product } \\
\text { Size } \\
999 \text { bp }\end{array}$} \\
\hline & SALK_049380 & $\begin{array}{l}\text { hd2b-1 } \\
\text { LP/RP }\end{array}$ & $\begin{array}{l}\text { 5'-AAGCTGATAC } \\
\text { CGAAACCAAGC- } \\
\text { '3 }\end{array}$ & $\begin{array}{l}\text { 5'-GATACCGCAT } \\
\text { CAGCTTCAATC- } \\
\text { '3 }\end{array}$ & \\
\hline & SALK_113500 & $\begin{array}{l}\text { hd2b-2 } \\
\text { LP/RP }\end{array}$ & $\begin{array}{l}\text { 5'-TACCCCAGAA } \\
\text { CTCCATTGTTG-'3 }\end{array}$ & $\begin{array}{l}\text { 5'-ATGATGATGA } \\
\text { TGATCTTGGGC- } \\
\text { '3 }\end{array}$ & $1098 \mathrm{bp}$ \\
\hline & $\begin{array}{l}\text { SAIL_1247_A } \\
02\end{array}$ & $\begin{array}{l}\text { hd2b-3 } \\
\text { LP/RP }\end{array}$ & $\begin{array}{l}\text { 5'- TCTTCTTCTC } \\
\text { CTCCCGATAGC-'3 }\end{array}$ & $\begin{array}{l}\text { 5'- AACAAGTTTA } \\
\text { GCCCCACCAAC } \\
-3\end{array}$ & $1137 \mathrm{bp}$ \\
\hline \multirow[t]{7}{*}{ HD2D } & SALK_104071 & $\begin{array}{l}\text { hd2d-1 } \\
\text { LP/RP }\end{array}$ & $\begin{array}{l}\text { 5'- CAGAGAAAGC } \\
\text { GATGTCGTTTC-3' }\end{array}$ & $\begin{array}{l}\text { 5'- GGCTTATTTTT } \\
\text { GAATCGG-3' }\end{array}$ & $1100 \mathrm{bp}$ \\
\hline & SALK_095273 & $\begin{array}{l}\text { hd2d-2 } \\
\text { LP/RP }\end{array}$ & $\begin{array}{l}\text { 5' AATCTGAACC } \\
\text { GTCACGATTTG-'3 }\end{array}$ & $\begin{array}{l}\text { 5'-CAGAAGAAA } \\
\text { GGCAAGCTTTT } \\
\text { G-3 }\end{array}$ & $1173 \mathrm{bp}$ \\
\hline & GT_5_100004 & $\begin{array}{l}\text { hd2d-3 } \\
\text { LP/RP }\end{array}$ & $\begin{array}{l}\text { 5'-CTCGCAGTTT } \\
\text { AGTCTTGAGAGT } \\
\text { ATTG-'3 }\end{array}$ & $\begin{array}{l}\text { 5'-AAATGATTA } \\
\text { ACTTTAATGGC } \\
\text { GAAAAC-'3 }\end{array}$ & $1173 \mathrm{bp}$ \\
\hline & GT_5_100010 & $\begin{array}{l}\text { hd2d-4 } \\
\text { LP/RP }\end{array}$ & $\begin{array}{l}\text { 5'-CTCGCAGTTT } \\
\text { AGTCTTGAGAGT } \\
\text { ATTG-3 }\end{array}$ & $\begin{array}{l}\text { 5'-AAATGATTAA } \\
\text { CTTTAATGGCG } \\
\text { AAAAC-'3 }\end{array}$ & $1173 \mathrm{bp}$ \\
\hline & GABI_278D04 & $\begin{array}{l}\text { hd2d-5 } \\
\text { LP/RP }\end{array}$ & $\begin{array}{l}\text { 5'-TCAAAAGCAT } \\
\text { CAAAGGTCCTG-'3 }\end{array}$ & $\begin{array}{l}\text { 5' AGCCCACTA } \\
\text { AAAATAAGGCC } \\
C-3\end{array}$ & $1033 \mathrm{bp}$ \\
\hline & GABI_379G06 & $\begin{array}{l}\text { hd2d-6 } \\
\text { LP/RP }\end{array}$ & $\begin{array}{l}\text { 5'-GGGAGGTCTT } \\
\text { GATTGGTTTACTC } \\
-3\end{array}$ & $\begin{array}{l}\text { 5'- ATCAGGACA } \\
\text { GTCCAAGCCAA } \\
\text { G-'3 }\end{array}$ & $1190 \mathrm{bp}$ \\
\hline & GABI_836B08 & $\begin{array}{l}\text { hd2d-7 } \\
\text { LP/RP }\end{array}$ & $\begin{array}{l}\text { 5'-TCATTGGCTC } \\
\text { AAATTTATGGG-'3 }\end{array}$ & $\begin{array}{l}\text { 5'-CACAAAGATA } \\
\text { GATGCTTTGAC } \\
\text { ACC'-3 }\end{array}$ & $1210 \mathrm{bp}$ \\
\hline
\end{tabular}


The T-DNA insertion for the HD2B T-DNA Stocks were confirmed using the hd2b-1 LP primer for SALK_049380, hd2b-2 LP primer for SALK_113500, and the SALKLba1 primer which were designed from the left border (LB) of the TDNA. The hd2b-3 LP primer and the SAILLB1 (left border of the T-DNA) primer were used for SAIL_1247_A02.

The T-DNA insertion for the HD2D T-DNA Stocks were confirmed using the hd2d-1 LP primer for SALK-104071 with the SALKLba1 primer, the hd2d-2 LP primer for SALK-095273 with the SALKLba1 primer, the hd2d-3 LP primer for GT-5-100004 with the Ds3-1 primer, the hd2d-4 LP primer for GT-5-100010 with the Ds3-1 primer, the hd2d-5 RP primer for GABI_278D04 with the GABILB primer, the hd2d-6 LP primer with the GABILB primer for the GABI_379G06 and the hd2d-7 LP primer with the GABILB primer for the GABI_836B08 (Table 8). 
Table 8: Verification of T-DNA Insertion Using LB and RP/LP Primers

\begin{tabular}{|l|l|l|l|}
\hline $\begin{array}{l}\text { Left T-DNA } \\
\text { boarder } \\
\text { primer LB }\end{array}$ & $\begin{array}{l}\text { Primer } \\
\text { Name }\end{array}$ & Primer Sequence & $\begin{array}{l}\text { BP+RP/LP } \\
\text { Product } \\
\text { size }\end{array}$ \\
\hline $\begin{array}{l}\text { LB for } \\
\text { SALK lines }\end{array}$ & $\begin{array}{l}\text { SALKLba } \\
1\end{array}$ & 5'- TGGTTCACGTAGTGGGCCATCG-'3 & $400+N^{1}$ \\
\hline $\begin{array}{l}\text { LB for } \\
\text { SAlL lines }\end{array}$ & SAILLB1 & $\begin{array}{l}\text { 5'GCCTTTTCAGAAATGGATAAATAGCC } \\
\text { TTGCTTCC-'3 }\end{array}$ & $400+N$ \\
\hline $\begin{array}{l}\text { LB for } \\
\text { GABI lines }\end{array}$ & GABILB & 5'-ATATTGACCATCATACTCATTGC-'3 & $400+N$ \\
\hline $\begin{array}{l}\text { LB for SIM } \\
\text { lines }\end{array}$ & Ds3-1 & 5'-ACCCGACCGGATCGTATCGGT-3' & $400+N$ \\
\hline
\end{tabular}

$1 \quad \mathrm{~N}-$ Is the difference of the actual insertion site and the flanking sequence position, usually $0-300$ bases. 
2.2.3 Loss-of-function Confirmation by Semi-Quantitative Reverse-Transcription PCR (RT-PCR)

Semi-quantitative reverse transcription PCR (RT-PCR) was performed to determine the relative gene expression level of $H D 2 B$ and $H D 2 D$ in the T-DNA homozygous insertion lines compared to respective wild-types ecotypes. Total RNA was harvested from 7 day old seedlings mutants lines and wild-type ecotypes grown on solid media (as previously described-Growth-Conditions). Approximately $100 \mathrm{mg}$ of plant tissue was harvested into $2 \mathrm{ml}$ RNase-free microcentrifuge tube and directly frozen in liquid nitrogen. RNA was then extracted using the RNeasy Plant Mini Kit (Qiagen, Hilden, Germany) according to the manufacturer's protocol. Frozen tissues were homogenized with a Eurostar power-b tissue homogenizer (Eurostar IKA-Labotechnik, Staufen, Germany). $450 \mu l$ RLT buffer (Lysis buffer, contains guanidine thiocyanate and $10 \mu \mathrm{l}$ of $\beta$ Mercaptoethanol) was added and tissue were further homogenized to form a fine homogenate. The solution was mixed by vortexing and incubated at $56^{\circ} \mathrm{C}$ for 3 min to disrupt the tissue. The lysate was then transferred to a QIAshredder spin column (Qiagen, Hilden, Germany) placed in a $2 \mathrm{ml}$ collection tube, and centrifuged for $2 \mathrm{~min}$ at full speed $(13,000 \mathrm{rpm})$. The supernatant of the flowthrough was transferred to a new microcentrifuge tube without disturbing the celldebris pellet in the collection tube. Then, 0.5 volume of ethanol (95\%) was added to the cleared lysate, and immediately mixed by pipetting and transferred to an RNeasy spin column placed in a $2 \mathrm{ml}$ collection tube and centrifuged for 15

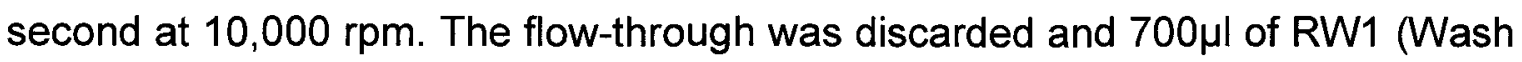


buffer, contains ethanol and a small amount of guanidine thiocyanate) was added to the RNeasy spin column and centrifuged for $15 \mathrm{sec}$ at $10,000 \mathrm{rpm}$ to wash the spin column membrane. The flow-through was discarded and $500 \mu l$ of buffer RBE (Wash buffer contains ethanol) was added to the RNeasy spin column and centrifuged for 15 second at $10,000 \mathrm{rpm}$ to wash the spin column membrane. The flow-through was then discarded and another $500 \mu$ l of RBE buffer to the RNeasy spin column and centrifuged for 2 minutes at $10,000 \mathrm{rpm}$ to wash the spin column membrane. The flow-through was discarded and the RNeasy spin column was centrifuged at full speed $(13,000 \mathrm{rpm})$ for $1 \mathrm{~min}$. The RNeasy spin column was placed into a new $1.5 \mathrm{ml}$ collection tube and $50 \mu \mathrm{l}$ of RNase-free water was added directly to the spin column membrane, incubated at room temperature for 1 minute and centrifuged for 1 minutes at $10,000 \mathrm{rpm}$ to elute the RNA.

The concentration of RNA was then measured using Nanodrop ND-2000 spectrophotometer (Thermo Fisher Scientific, Wilmington, Delaware, USA). One microgram of total RNA was then used for the one step RT-PCR (Qiagen, GmbH, Hilden) according to manufacture's protocol to assess the presence of a truncated $H D 2 B$ mRNA transcript in the homozygous $H D 2 B$ T-DNA insertion line by using two primers upstream of the mapped insertion. The primers used during the one step RT-PCR were A.HD2B LP and A.HD2B RP (Table 9, Figure 4) for the truncated mRNA, and B.HD2B LP and B. HD2B RP (Table 9, Figure 4) that are downstream of the insertion site were used to detect the full-length transcript. The primers were designed to anneal to exon junctions to eliminate amplification 
of genomic DNA contamination. The annealing temperature for each reaction was $59^{\circ}$ Celsius with 40 cycles of amplification:

The same strategy was used for the homozygous HD2D T-DNA insertion line. Primers used to asses the presence of a truncated $H D 2 D$ mRNA transcript were A.HD2D LP/A'.HD2D, A.HD2D RP/A'.HD2D (Table 9, Figure 5) and the primers used to detect the full-length transcript were B.HD2D LP and B.HD2D RP (Table 9, Figure 5). The annealing temperature for each reaction was $57^{\circ}$ Celsius with 40 cycles of amplification.

Table 9: Detection of full length and short length mRNA

\begin{tabular}{|l|l|l|l|l|}
\hline Gene & $\begin{array}{l}\text { Primer } \\
\text { Name }\end{array}$ & Left Primer (LP) & Right Primer (RP) & $\begin{array}{l}\text { Product } \\
\text { Size }\end{array}$ \\
\hline HD2B & $\begin{array}{l}\text { A.HD2B } \\
\text { LP/RP }\end{array}$ & $\begin{array}{l}\text { 5'-AAAATAGCCCCAAA } \\
\text { CCCACT-'3 }\end{array}$ & $\begin{array}{l}\text { 5'-CCTCGTCTGATTCAG } \\
\text { GCTTC-'3 }\end{array}$ & $570 \mathrm{bp}$ \\
\cline { 2 - 5 } $\begin{array}{l}\text { B.HD2B } \\
\text { LP/RP }\end{array}$ & $\begin{array}{l}\text { 5'-GACTCCTGAAGAAGA } \\
\text { CAGCCTT-3 }\end{array}$ & $\begin{array}{l}\text { 5'- TTGTTGTTACCACCG } \\
\text { GATGA-3 }\end{array}$ & $789 \mathrm{bp}$ \\
\hline HD2D & $\begin{array}{l}\text { A.HD2D } \\
\text { LP/RP }\end{array}$ & $\begin{array}{l}\text { 5'-ATTAAGCCAGGGAAG } \\
\text { CCATT-'3 }\end{array}$ & $\begin{array}{l}5^{\prime} \text {-AAGAGGGACCACAAG } \\
\text { GGAAT-'3 }\end{array}$ & $580 \mathrm{bp}$ \\
\cline { 2 - 5 } & $\begin{array}{l}\text { A'.HD2D } \\
\text { LP/RP }\end{array}$ & $\begin{array}{l}\text { 5'-ATTAAGCCAGGGAAG } \\
\text { CCATT-3 }\end{array}$ & $\begin{array}{l}\text { 5'-CATCTTTTTGCTCG } \\
\text { GAGGAG-'3 }\end{array}$ & $489 \mathrm{bp}$ \\
\cline { 2 - 5 } & $\begin{array}{l}\text { B.HD2D } \\
\text { LP/RP }\end{array}$ & $\begin{array}{l}5^{\prime} \text {-AGCCGCTCCATTATT } \\
\text { TTTG -'3 }\end{array}$ & $\begin{array}{l}\text { 5'-AGGGAATGGGCATCT } \\
\text { CTTCT-3 }\end{array}$ & $687 \mathrm{bp}$ \\
\hline
\end{tabular}


Figure 4: Map of HD2B T-DNA insertion lines and designated primers for truncated mRNA $(A)$ and full length mRNA $(B)$ detection. A.HD2B_LP represents primers that are designed to detect transcript after the insertion event, while B.HD2B_LP represents primers designed to detect transcript before the insertion event. T-DNA triangle represents the site of T-DNA insertion for each SALK line.
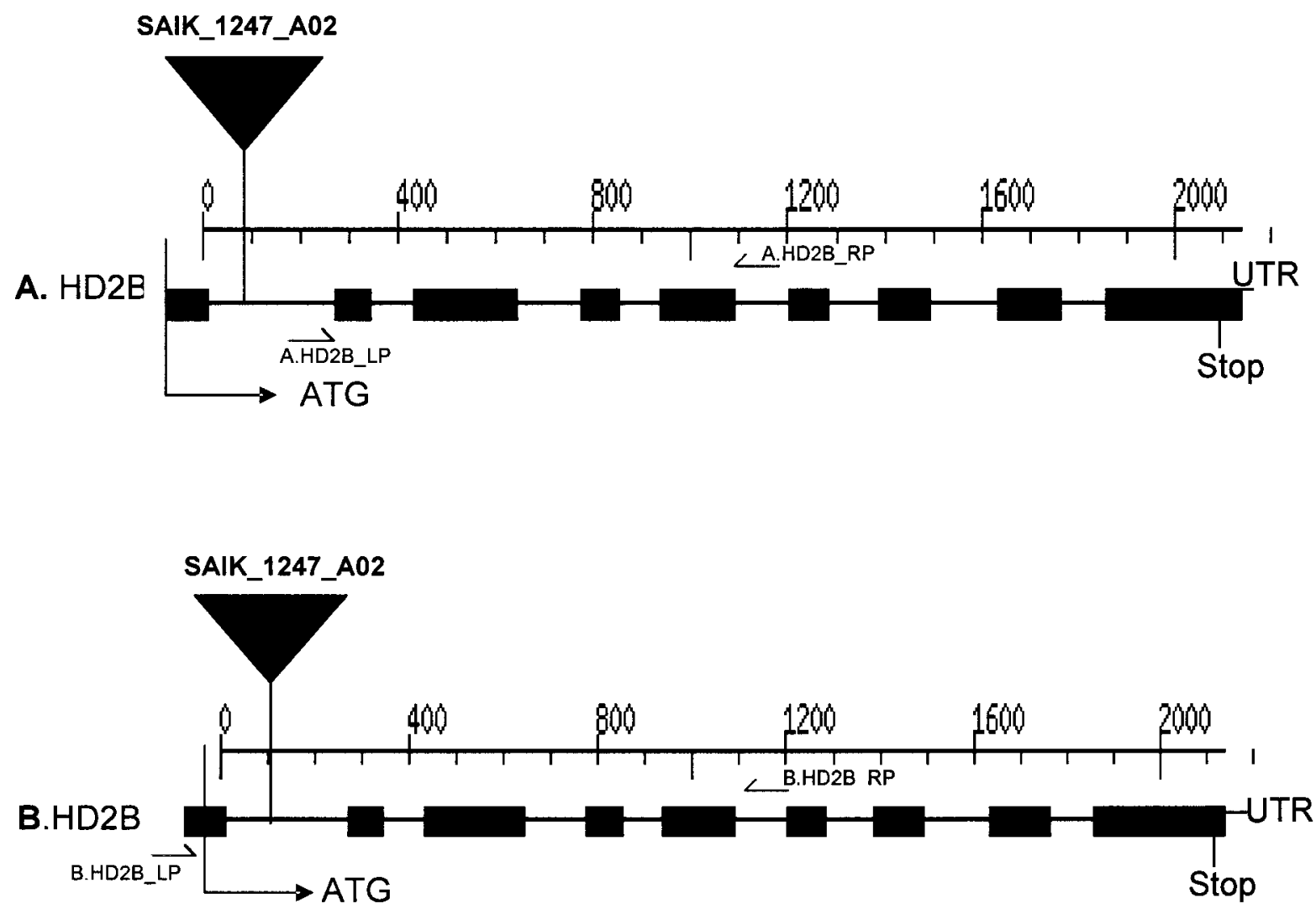

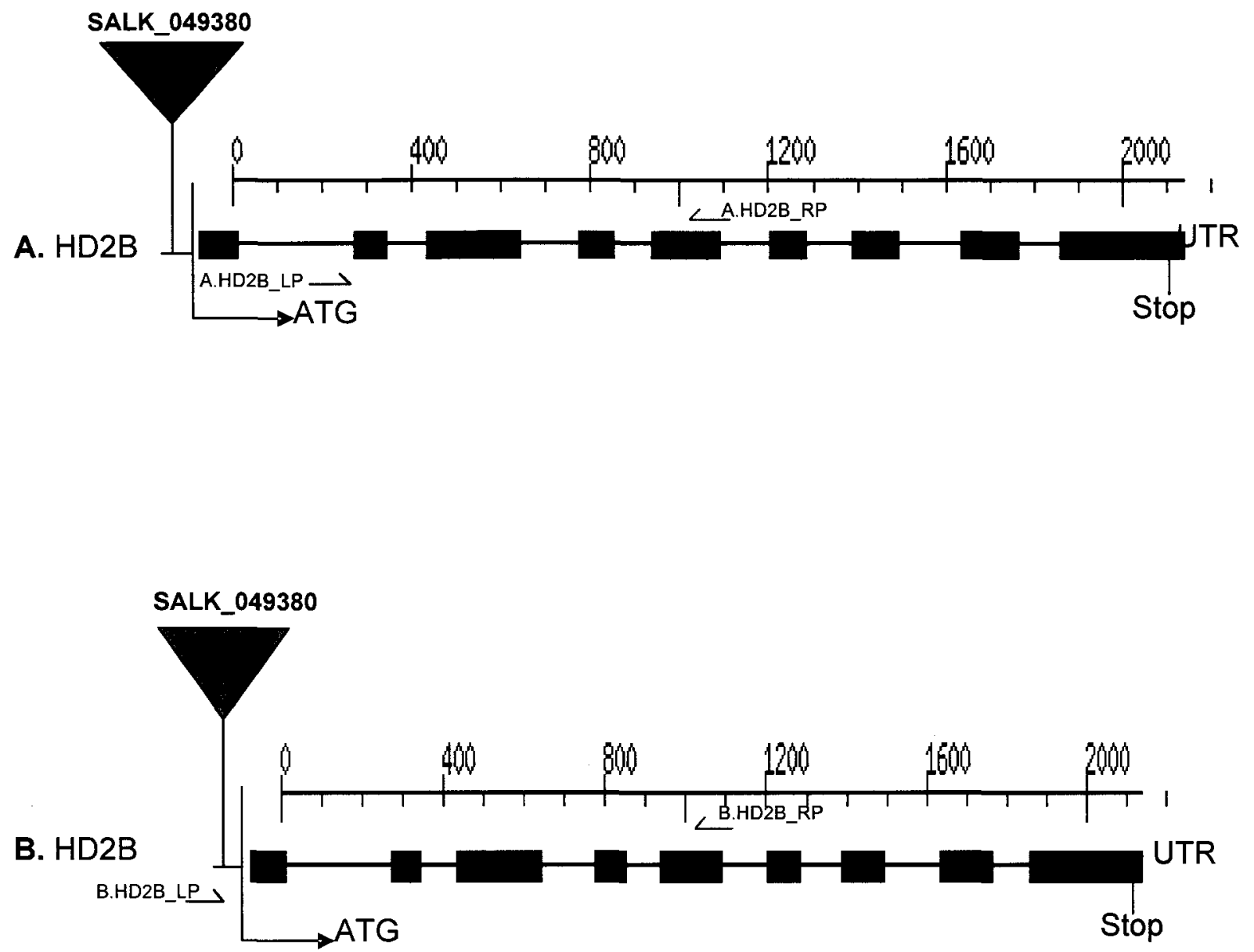

SALK_113500

A. HD2B

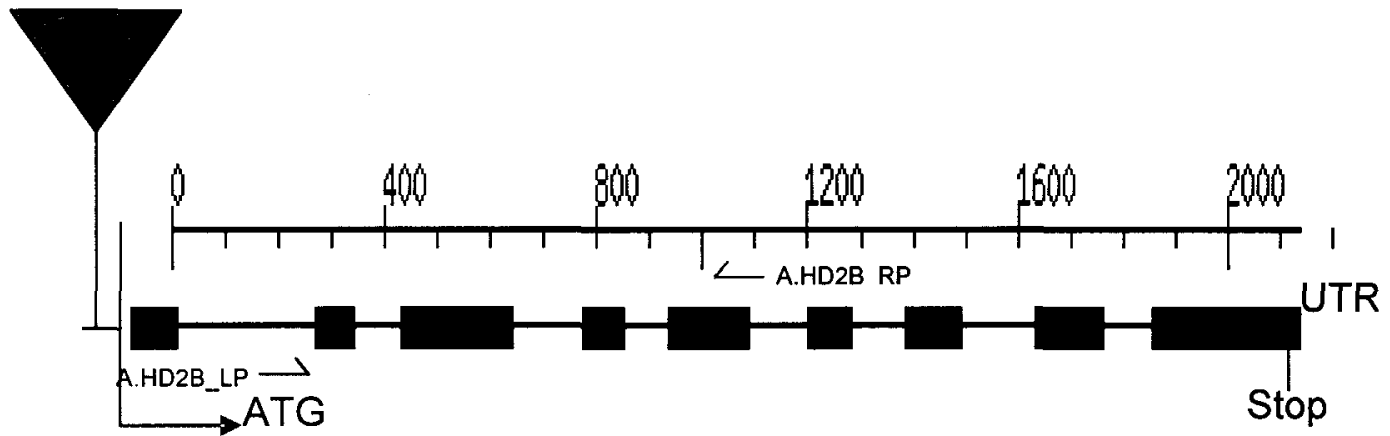




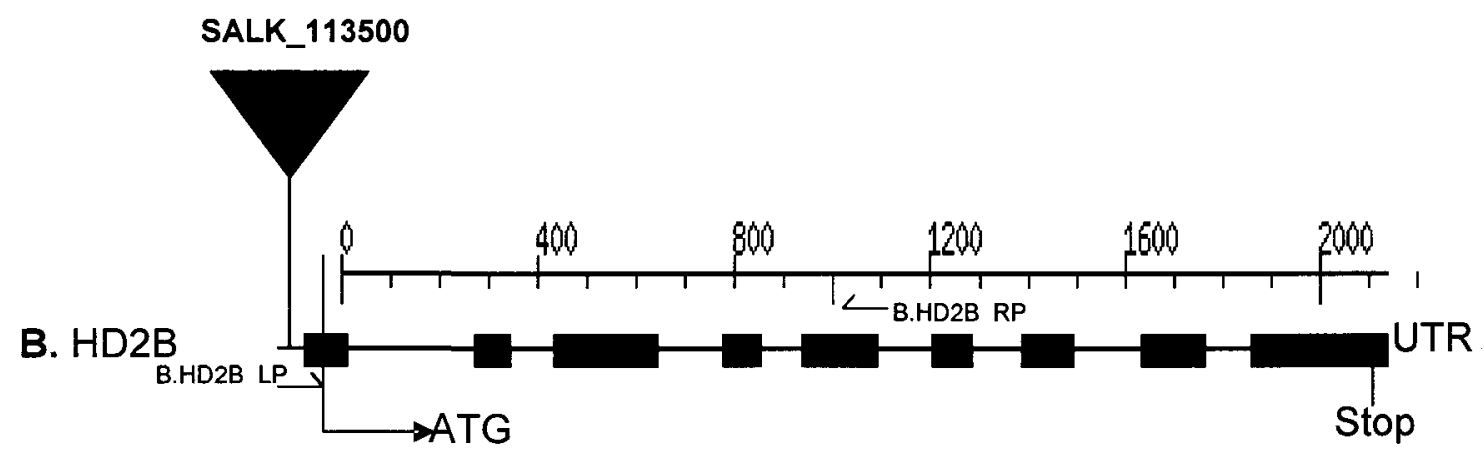


Figure 5: Map of HD2D T-DNA Insertion lines and designated primers for truncated mRNA $(A)$ and full length mRNA (B) detection. A.HD2D_LP represents primers that are designed to detect transcript after the insertion event, while B.HD2D_LP represents primers designed to detect transcript before the insertion event. T-DNA triangle represents the site of T-DNA insertion for each SALK line. .
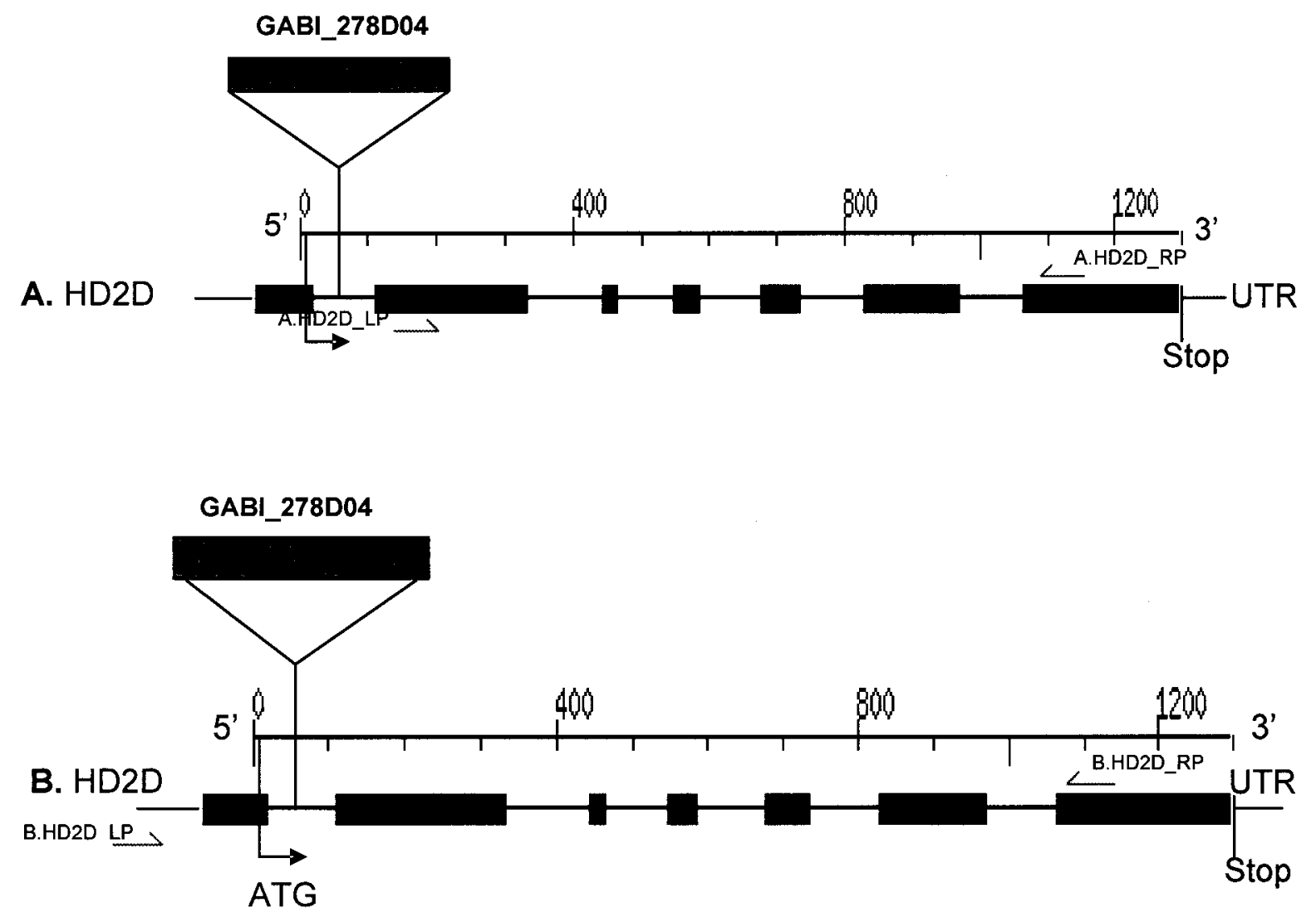

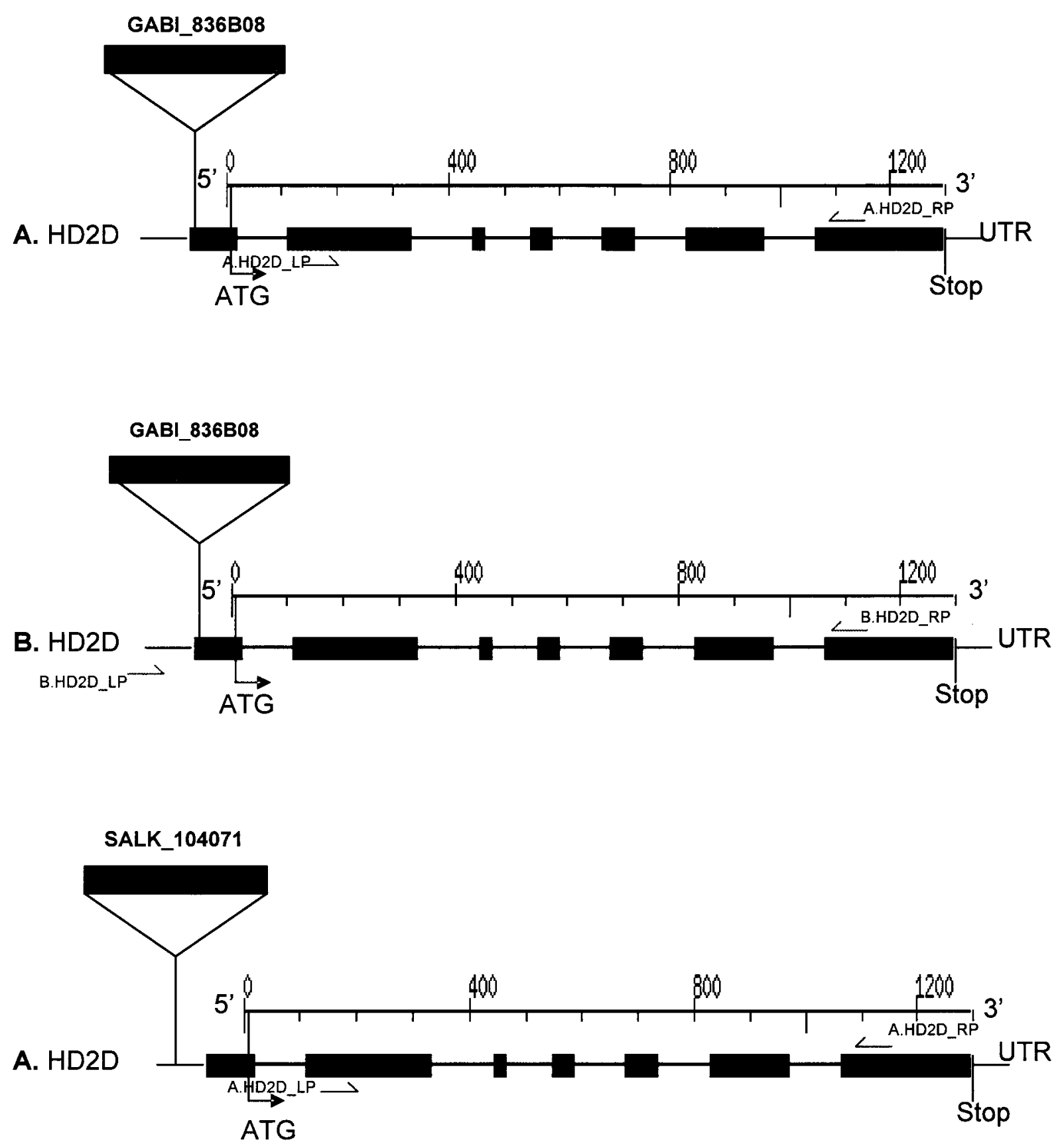

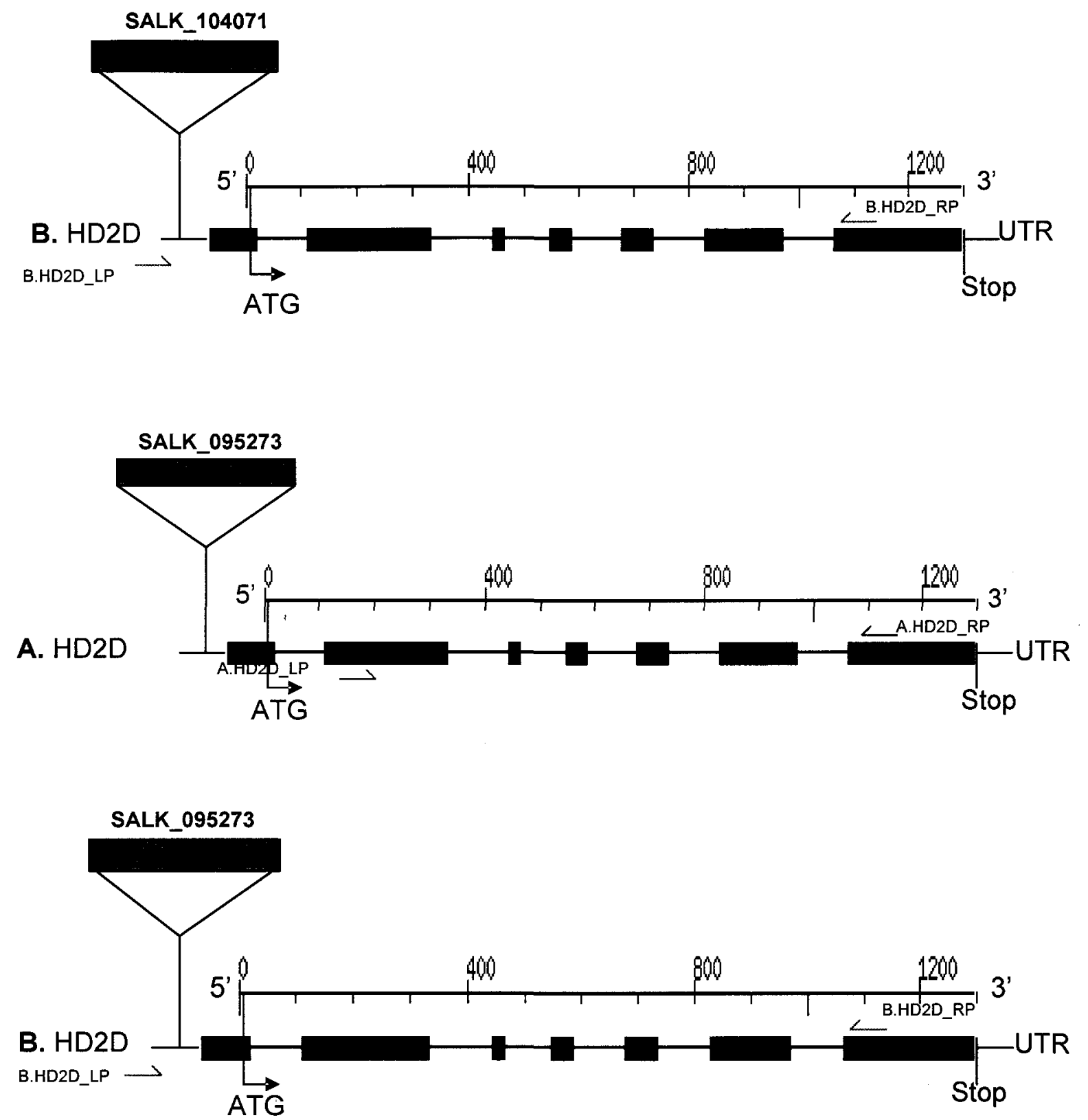

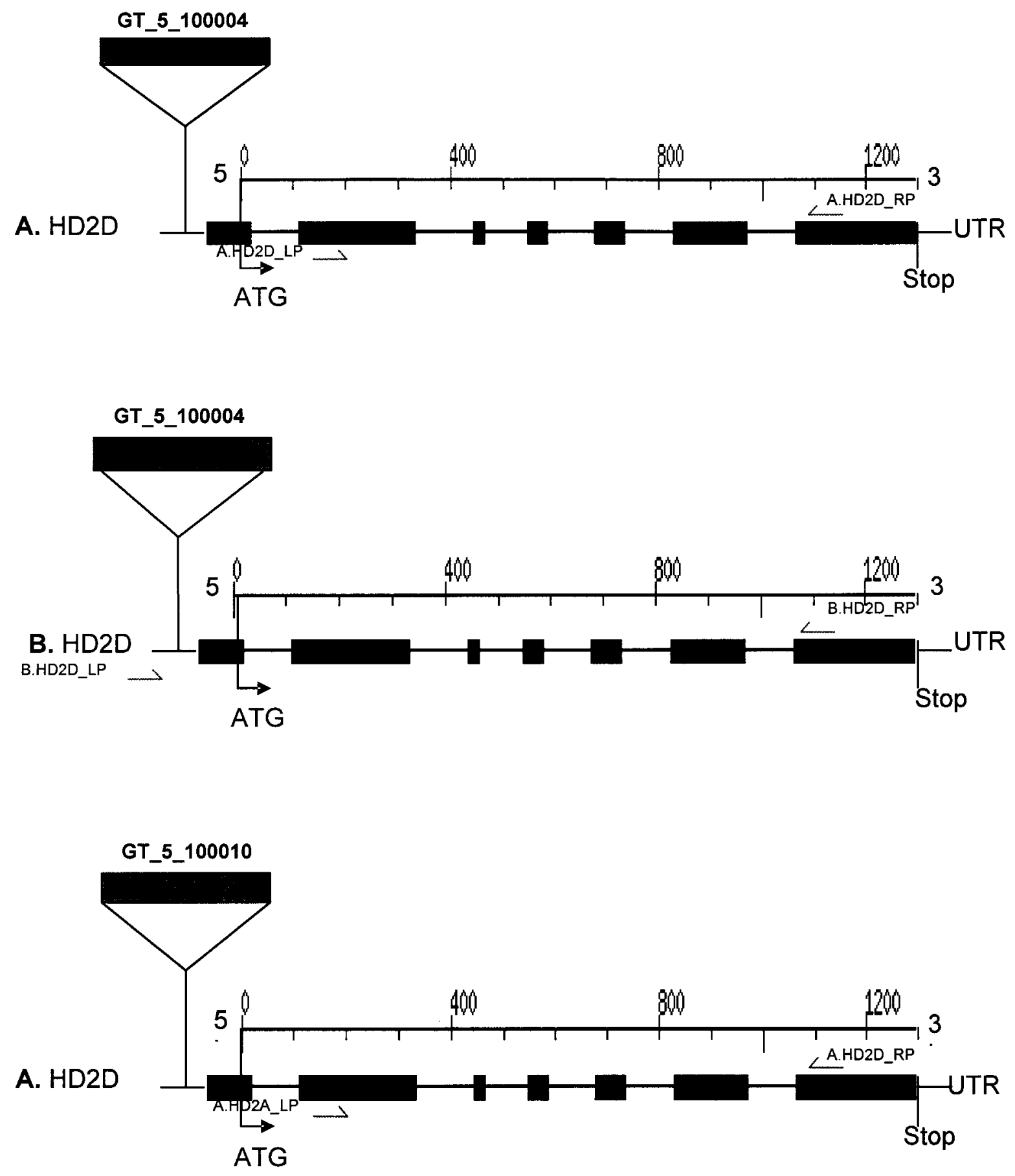

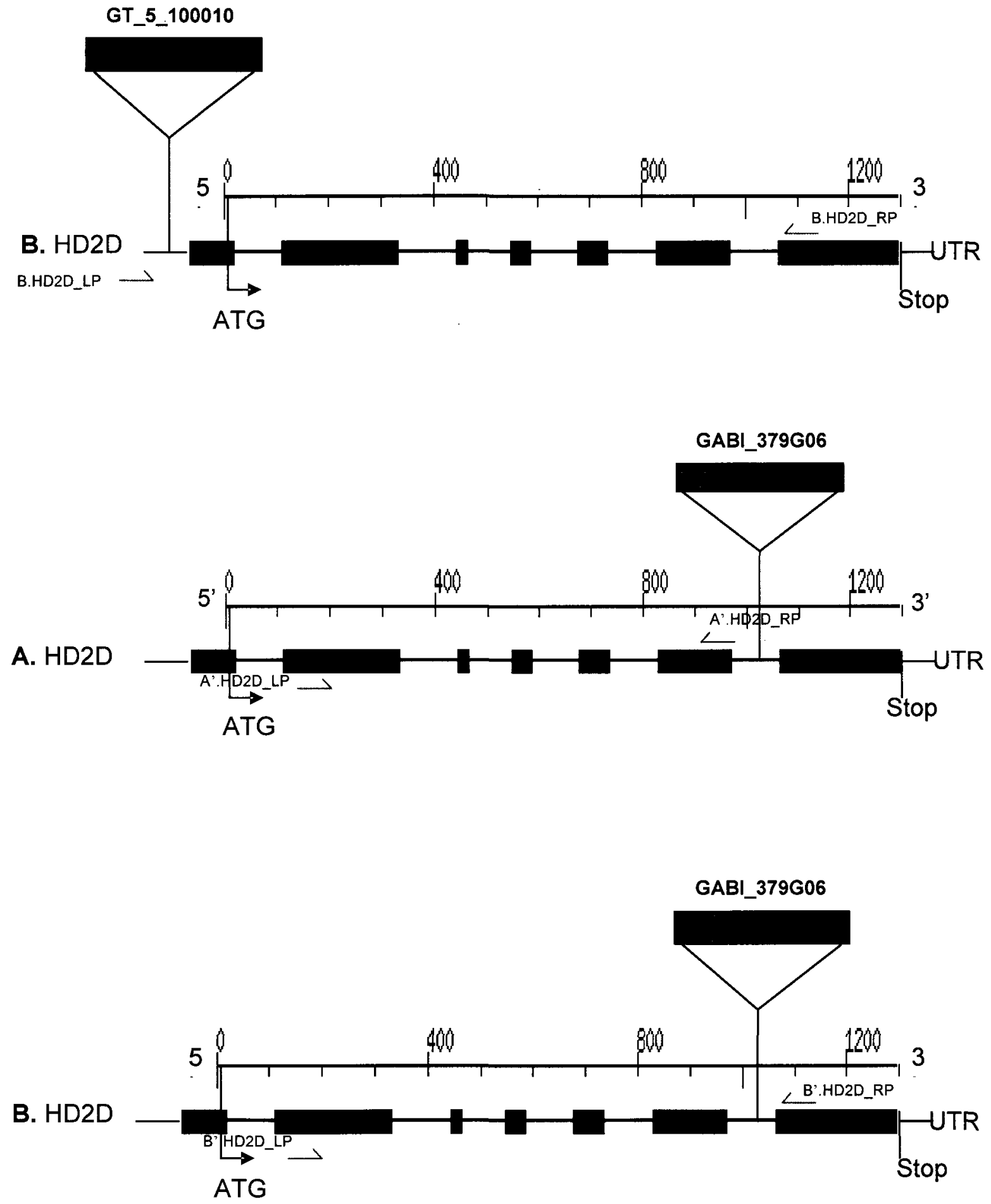
2.3 Induction of HD2 Gene Expression upon Glucose Treatment

Arabidopsis thaliana ecotype Columbia (Col) seeds were surfacesterilized and stratified as previously described. Seeds were sown on $1 / 2 \mathrm{MS}$, $0.8 \%(\mathrm{w} / \mathrm{v})$ Phytagar, $\mathrm{pH} 5.7$ glucose-free media on dialysis membrane backing (BIO-RAD, California USA). Seeds were then allowed to germinate under continuous light for 24 hours. Plants were then incubated in the dark for 4 days. Plates were then incubated with $15 \mathrm{ml} \mathrm{H} 2 \mathrm{O}$, or $3 \%(w / v)$ glucose $(166 \mathrm{mM})$ solution for $2,4,6,8,10,12$, and 16 hours in the dark. $100 \mathrm{mg}$ of treated seedlings were then collected and RNA was then harvested as previously described. Two microgram of total RNA was then transcribed into cDNA as described previously. Reverse-Transcription-PCR (RT-PCR) was then carried out on the HD2 gene family with the same PCR parameters as previously described. The primers employed for checking the transcript of HD2A were HD2A_FW_primer (5'-ATGGAGTTCTGGGGAATTGAAG-'3) and HD2A_RV_primer (5'- ACATGAGACTTGACTGGCCGAC-'3), for HD2B were HD2B_FW_primer (5'-ATGGAGTTCTGGGGAGTTGC-'3), and HD2B_RV_primer )5'-TGTTTGCCTGAGTTGAATGG-'3), for HD2C were HD2C_FW_primer (5'- CAGGTTGCTTTGGGAGAGAG-'3) and HD2C_RV_primer (5'-TCAAGCAGCTGCACTGTGTTTG-'3), and HD2D were HD2D_FW_primer (5'-ATGGAGTTTTGGGGTATCG-'3) and HD2D_RV_primer (5'-AGCCTCATAAGTCACTCAACGA-'3). 
2.4 Transcriptional compensation RT-PCR analysis by RT-PCR

$H D 2 A, H D 2 B$, and $H D 2 D$ genes were analyzed for potential transcriptional down-regulation as a compensatory mechanism for constitutive expression of HD2C. Approximately $100 \mathrm{mg}$ of plant tissues were harvested, RNA extracted and quantified as previously described. RNA quality was then checked by running $2 \mu \mathrm{l}$ of total RNA on a 1.2\% agarose, 1XMOPS (4morpholinopropanesulfonic) buffer RNA gel. First strand of cDNA synthesis was then transcribed by SuperScript III first cDNA synthesis system (Invitrogen, Carlsbad, CA, USA) according to the manufacture's protocol. Two micrograms of total RNA in the presence of a $1 \mu \mathrm{l}$ poly-T2o primer, $1 \mu \mathrm{l} 10 \mathrm{mM}$ dNTP mix, and up to $11 \mu \mathrm{l}$ of sterilized water were incubated at $65^{\circ} \mathrm{C}$ for 5 minutes. At $20^{\circ} \mathrm{C}, 4 \mu \mathrm{l} 5 \mathrm{x}$ first strand cDNA synthesis buffer, $1 \mu \mathrm{l} 0.1 \mathrm{M}$ DTT, $1 \mu \mathrm{l}$ sterilized water, and $1 \mu \mathrm{l}$ Superscript III were added to each sample, and then incubated 60 minutes at $50^{\circ} \mathrm{C}$, and then 15 minutes at $70^{\circ} \mathrm{C}$. The primers used to amplify $H D 2 A$ mRNA transcript were HD2A_FW (5'- ATGGAGTTCTGGGGAATTGAAG-'3) and HD2A_RV primer (5'- ACATGAGACTTGACTGGCCGAC-'3). The amplification of HD2B mRNA transcript was carried out with HD2B_FW (5'ATGGAGTTCTGGGGAGTTGC-'3) and HD2B_RV primer (5'TGTTTGCCTGAGTTGAATGG-'3). The primers employed to amplify HD2D mRNA transcript were HD2D_FW (5'- ATGGAGTTTTGGGGTATCG-'3) and HD2B_RV (5'- AGCCTCATAAGTCACTCAACGA-3). An internal control, $\beta$ TUBULIN was used to ensure accuracy and effectiveness of the reaction using the gene specific primer, $\quad \beta$-TUBULIN_FW primer 
GGGCTAAAGGACACTACACTGAAGG-'3) and $\beta$-TUBULIN_RV primer (5'CCTCCTGCACTTCCACTTCGTCTTC-'3). $2 \mu \mathrm{l}$ of CDNA sample was used to run PCR. Samples were incubated at $94^{\circ} \mathrm{C}$ for 2 minutes, $94^{\circ} \mathrm{C}$ for 30 seconds, $59^{\circ} \mathrm{C}$ (depending on primer) for 30 seconds, $70^{\circ} \mathrm{C}$ for 1 minute for 25 cycles. All the PCR products were run on a $1.2 \%(\mathrm{w} / \mathrm{v})$ agarose gel electrophoresis. Gel images were photographed using a Molecular Imager chemiDoc XRS system (Bio-Rad, Hercules, CA, USA).

Furthermore, single $h d 2 a$ and $h d 2 c$ loss of function mutants, as well as a double $h d 2 a$ hd2c mutant were analyzed for potential transcriptional compensation among these members upon glucose induction. Seeds were germinated in the absence of sucrose and presence of light for 24 hour. Plants were then covered with tin foil. After 4 days, a $3 \%$ glucose $(166 \mathrm{mM})$ solution was added to the plate and total RNA was harvested after 4 hours of exposure in the absence of light. RT-PCR of $H D 2 B$ and $H D 2 D$ transcript was examined for potential transcriptional up-regulation. Same set of primers as used for potential transcriptional down-regulation as a compensatory mechanism for constitutive expression of $H D 2 C$ were used. $\beta$-TUBULIN was used as an internal control. The same PCR conditions and parameters as used for potential transcriptional downregulation as a compensatory mechanism for constitutive expression of $H D 2 C$ GFP were used. 


\section{$2.5 H D 2$ and Sugar Signaling}

Arabidopsis thaliana ecotype Landsberg erecta (Ler) was used as the wild-type control for this study. Homozygous gin2 loss-of-function seeds in the Ler background were denoted by $\mathrm{Dr}$. Jen Sheen, Institution (Department of Molecular Biology-Massachusetts General Hospital). The phenotype of homozygous gin 2 seeds was confirmed by growing seeds on $6 \%$ glucose $(330$ $\mathrm{mM})$. Seeds were then sterilized and stratified as described before. Seeds were sown on $1 / 2 \mathrm{MS}, 0.8 \%(\mathrm{w} / \mathrm{v})$ Phytagar, $\mathrm{pH} 5.7$ glucose-free media (BIO-RAD, California USA). Seeds were germinated in continuous light for 24 hours. Plants were then removed from the continuous light and incubated in the dark for 4 days. Plates were then incubated with either $15 \mathrm{ml}$ of $\mathrm{H}_{2} \mathrm{O}$ or $3 \%(w / v)$ glucose (166 $\mathrm{mM}$ ) solution for 4 hours in the absence of light. RNA was then harvested as described previously. Induction of the HD2 members by $3 \%$ glucose (166 mM) was then assessed by Reverse Transcription-PCR (RT-PCR) using gene specific primers in the control and the mutant background. This process was repeated with other soluble carbohydrates (fructose, sucrose, glucose-6-phosphate, fructose-6-phosphate, mannitol, and 3-O-methyl-D-glucose). PCR parameters were the same as previously described. The primers used for amplification of HD2A transcript were qHD2A_LP_Primer (5'-TGATTCTGACGGAATGGATG-'3) and qHD2A_RP_Primer (5'- CCTGAGTTGAAAGTCTTCTTGC-'3), for HD2B were qHD2B_LP_Primer (5'- GACTCCTAAGAAGCCTGAGCC-'3) and qHD2B_RP_Primer (5'- CTGAGTTGAATGGCTTCTTGT-'3), for HD2C were qHD2C_LP_Primer (5'-CTGGTTTCAAAGCTGCTCC-'3) and qHD2C_RP_Primer 
(5'- CGGTTTCTTCGAATCTGTTTTT-'3), for HD2D were qHD2D_LP_Primer (5'ACGAACTGGATGAGGAGATT-'3) and qHD2D_RP_Primer (5'CTCTTCTTCCCTCCTGATGA-'3), for the internal control $\beta$-TUBULIN were q $\beta$ TUBULIN_LP_Primer (5'ACTGCGATTGCCTTCAAG-'3) and $q \quad \beta$ TUBULIN_RP_Primer (5'-GTTCAAGTCTCCAAAGCTAGGA-'3).

2.6 GUS Histochemical Staining

An identical experimental approach as used for the induction of $H D 2$ gene expression by soluble carbohydrates was used to examine the spatial expression patterns conferred by the HD2 promoter. Transgenic AtHD2C::GUS tissues obtained from Keqiang Wu (Department of Biology, West Virginia University, Morgantown, WV 26506, USA) were submerged in 1mM 5-bromo-4-chloro-3indolyl-glucuronic acid solution in $100 \mathrm{mM}$ sodium phosphate $(\mathrm{pH} 7.0)$ with 10 mM EDTA, $0.5 \mathrm{mM}$ ferricyanide, $0.5 \mathrm{mM}$ ferrocyanide and $0.1 \%$ Triton $\mathrm{X}-100$, vacuumed infiltrated for 30 minutes and incubated at $37^{\circ} \mathrm{C}$ overnight, followed by series of $30 \%, 50 \%$, and $70 \%$ ethanol washes for 2 hours each to remove the chlorophyll. Images were taken by WD 54 Nikon JAPAN microscope. This method was adopted from Wu et al. (2001).

\subsection{GUS Specific Activity}

An identical experimental strategy as used for the induction of $H D 2$ genes were used for HD2 promoter activity upon soluble carbohydrates treatment with transgenic seedlings expressing AtHD2C::GUS. $100 \mathrm{mg}$ of plant tissue was 
harvested and frozen in liquid nitrogen. Frozen tissues were then ground using a Eurostar power-b tissue homogenizer (Eurostar IKA-Labotechnik, Staufen, Germany). $300 \mu$ l of GUS extraction buffer was then added, and further homogenized until no large tissue pieces were left. Samples were then spun for 10 minutes at high speed in the cold room $\left(4^{\circ} \mathrm{C}\right)$. Supernatants were then transferred into $1.5 \mathrm{ml}$ screw cap tubes. $50 \mu$ of the crude extract was then added to $500 \mu \mathrm{l}$ pre-warmed assay buffer $(1 \mathrm{mM} 4$-methylumbelliferyl glucuronide in 0.3 $\mathrm{ml}$ of GUS assay buffer (50 mM NaPO $4, \mathrm{pH} 7.0,10 \mathrm{mM}$ EDTA, $0.1 \% \mathrm{v} / \mathrm{v}$ Triton $\mathrm{X}-100,10 \mathrm{mM} \beta$-mercaptoethanol) and incubated at $37^{\circ} \mathrm{C}$ with a $5 \mathrm{~min}, 30 \mathrm{~min}$, $60 \mathrm{~min}, 120 \mathrm{~min}$ intervals. $100 \mu \mathrm{l}$ aliquots were removed and added to $1.9 \mathrm{ml}$ of $\mathrm{Na}_{2} \mathrm{CO}_{3}$ stop buffer to stop the reaction. Standard curve for MU (7-hydroxy4methylcoumarin) calibration was prepared from $\mathrm{Na}_{2} \mathrm{CO}_{3}$ stop buffer and $10 \mu \mathrm{M}$ MU stock. Fluorescence was then determined by reading samples in the fluorometer. $5 \mu \mathrm{l}$ of the crude extract was used for protein determination (Bradford Assay). Standards were prepared from BSA diluted in GUS Extraction buffer. Stocks of $0,0.2,0.4,1.0,1.5$ and $2.0 \mu \mathrm{gBSA} / \mu \mathrm{l}$ extraction buffer were prepared and $10 \mu \mathrm{l}$ of each stock was added to $1 \mathrm{ml}$ of $1: 5$ diluted Bradford reagent to provide a standard of $2,4,8,10,15$, and $20 \mu \mathrm{g} / \mathrm{ml}$ samples. Samples were then read in the spectrophotometer (Biomate3, Thermo Electron Corporation, Waltham, MA, USA). GUS activity was expressed as pmol 4methylumbelliferone/ $\mathrm{\mu g}$ protein/min. This method was described by Jefferson, (1987). 
2.8 Quantification of HD2 Transcript Level upon Soluble Carbohydrate Treatment by Real-Time PCR Method

Arabidopsis thaliana ecotype Columbia (Col-0) and Landsberg erecta (Ler) seeds and $h d 2$ mutants ( $h d 2 a, h d 2 c$ and $h d 2 a h d 2 c$ ) and gin2-1 null mutant as well as $35 \mathrm{~S}: \mathrm{HD} 2 \mathrm{~A}$ seeds were surface-sterilized and stratified as previously described. Seeds were sown on $1 / 2$ MS, $0.8 \% b(w / v)$ Phytagar, pH 5.7 glucose free media on dialysis membrane backing (BIO-RAD, California USA). Seeds were germinated in continuous light for 24 hours and plants were then removed from the continuous light and incubated in the dark for 4 days followed by the incubation with either $15 \mathrm{ml}$ of $\mathrm{H}_{2} \mathrm{O}$ or $3 \%(\mathrm{w} / \mathrm{v})$ soluble carbohydrate solution (glucose, fructose, sucrose, glucose-6-phosphate, fructose-6-phosphate, mannitol, and 3-O-methylglucose) for 4 hours in the absence of light as previously described. RNA was then harvested as described previously and DNA contamination was removed from the RNA samples by Deoxyribonuclease I, Amplification Grade (Invitrogen, Carlsbad, CA, USA). Up to $1 \mu \mathrm{g}$ of RNA sample, $1 \mu \mathrm{l} 10 \mathrm{x}$ DNase I reaction buffer, $1 \mu$ INase (Amplification Grade, $1 \mathrm{U} / \mu \mathrm{l}$ ), and miliqi-water bringing the sample volume to $10 \mu$ was incubated for 15 minutes at room temperature. The reaction was then inactivated by adding $1 \mu \mathrm{l}$ of $25 \mathrm{mM}$ EDTA ( $\mathrm{pH} \mathrm{8.0)}$ and heating the samples at $65^{\circ} \mathrm{C}$ for 10 minutes. One microgram of cDNA synthesis was then followed as previously described. Real-time PCR was then done in a BioRad MyiQ Single Color Real-Time PCR Detection System using Platinum $®$ SYBR $®$ Green qPCR SuperMix-UDG (Invitrogen, Carlsbad, CA, USA). Each well in the PCR plate contained $12.5 \mu$ Sybr green, $0.5 \mu$ l of $10 \mathrm{mM}$ 
forward primer, $0.5 \mu \mathrm{l}$ of $10 \mathrm{mM}$ reverse primer, $0.5 \mu \mathrm{l}$ ROX reference dye, $2 \mu$ l of 10x diluted CDNA, and $9 \mu \mathrm{LEPC}$-treated water. Samples were incubated at $50^{\circ} \mathrm{C}$ for 2 minutes, $95^{\circ} \mathrm{C}$ for 2 minutes, $95^{\circ} \mathrm{C}$ for 15 seconds, $57^{\circ} \mathrm{C}$ for 30 seconds, $72^{\circ} \mathrm{C}$ for 30 seconds for 40 cycles; 5 minutes at $72^{\circ} \mathrm{C}$. Real-Time data was taken relative to expression with $\beta$-TUBULIN primers.

Primers used to amplify HD2A-D and $\beta$-TUBULIN sequences in real-time PCR are listed in table 10.

Table 10: Primers used for Real-Time PCR

\begin{tabular}{|c|c|c|c|}
\hline Gene & Forward Primer & Reverse Primer & $\begin{array}{l}\text { Product } \\
\text { Size }\end{array}$ \\
\hline $\mathrm{HD} 2 \mathrm{~A}$ & $\begin{array}{l}\text { 5'-TGATTCTGACGGA } \\
\text { ATGGATG-'3 }\end{array}$ & $\begin{array}{l}\text { 5'- CCTGAGTTGAAAG } \\
\text { TCTTCTTGC-'3 }\end{array}$ & $261 \mathrm{bp}$ \\
\hline HD2B & $\begin{array}{l}\text { 5'- GACTCCTAAGAAG } \\
\text { CCTGAGCC-3 }\end{array}$ & $\begin{array}{l}\text { 5'- CTGAGTTGAATGG } \\
\text { CTTCTTGT-3 }\end{array}$ & $263 b p$ \\
\hline HD2C & $\begin{array}{l}\text { 5'-CTGGTTTCAAAGC } \\
\text { TGCTCC-'3 }\end{array}$ & $\begin{array}{l}\text { 5'- CGGTTTCTTCGAA } \\
\text { TCTGTTTT-'3 }\end{array}$ & $353 \mathrm{bp}$ \\
\hline HD2D & $\begin{array}{l}\text { 5'- ACGAACTGGATGA } \\
\text { GGAGATT-'3 }\end{array}$ & $\begin{array}{l}\text { 5'- CTCTTCTTCCCTC } \\
\text { CTGATGA-'3 }\end{array}$ & $277 b p$ \\
\hline$\beta$-TUBULIN & $\begin{array}{l}\text { 5'-ACTGCGATTGCCT } \\
\text { TCAAG-'3 }\end{array}$ & $\begin{array}{l}\text { 5'- GTTCAAGTCTCCA } \\
\text { AAGCTAGGA-3 }\end{array}$ & $302 b p$ \\
\hline
\end{tabular}


2.9 Phenotypic Analysis on HD2 Loss-of-function Lines and Over-expression Line

To reveal the physiological function of the HD2 family, wild-type (Col-0), hd2a mutants, $h d 2 c$ mutants, $h d 2 a h d 2 c$ double mutants, and 35S::HD2A seeds were surface sterilized as previously described and sown on solid minimal media supplemented with $0 \%, 2 \%, 5 \%, 6 \%, 7 \%$ glucose; $0.1 \mu \mathrm{m}, 0.2 \mu \mathrm{m} \mathrm{ABA} ; 100 \mathrm{mM}$ $\mathrm{NaCl} ; 150 \mathrm{mM} \mathrm{NaCl}, 200 \mathrm{mM} \mathrm{NaCl}, 250 \mathrm{mM}$ mannitol and $300 \mathrm{mM}$ mannitol under continuous fluorescent light at 22 to $25^{\circ} \mathrm{C}$ for germination, cell division, cell expansion and abiotic stress assay. Germination (fully emerged radicle) was scored at various time points in triplicate. The effect of the different conditions on seed germination was assessed by scoring the number of seedlings (cotyledon development) in each plate after 4 days of plating the seeds (done in triplicate). The effect of the different conditions on root growth was assessed by measuring the root length after 5 days of germination (done in triplicate). 


\section{CHAPTER 3}

\section{RESULTS}

\subsection{Specificity of $H D 2$ Induction}

A previous study (Colville, 2007) linked HD2 expression to the plant herbicide, imidazolinone, which inhibits branch-chain amino acid synthesis (Ray, 1984; Shaner et al., 1984) and isoxaben which blocks cellulose synthesis in higher plants (Scheible et al., 2001) by microarray analysis. It was found that HDACs induced by imidazolinone and isoxaben were specific to the HD2 family as the expression level of the 14 other HDACs within the Arabidopsis thaliana genome were not altered.

The HD2 up-regulation by imidazolinone and isoxaben treatment led to the hypothesis of $H D 2$ induction by glucose since glucose is the precursor of both cellulose (structural sugar) and de novo amino acid synthesis in Arabidopsis thaliana (Buchanan et al., 2000). It was believed that a disruption of either pathway may lead to an increase in the intracellular glucose concentration (Colville, 2007).

A method was developed to starve tissues of sugar before reintroduction to test the hypothesis and validate the finding by semiquantitative RT-PCR (Colville, 2007). Plants were incubated in the dark to eliminate photosynthesis effect on internal glucose. Since sugar is transported in the form of sucrose and cleaved into glucose and fructose by cell wall invertase enzymes during normal plant growth and development, the possibility of other soluble carbohydrates altering HD2 expression was 
proposed and tested by RT-PCR (Colville, 2007). However, previous data of Colville (2007) and Li et al., (2006) did not demonstrate if there was induction specificity among the HD2 family members. Thus, this study repeated the previous work comparing the relative level of induction of the individual $H D 2$ members using quantitative real-time PCR. Water was used as a control for experimental variation and minimization of background while mannitol was used as a control for osmotic stress.

3.2 Glucose Induction of the $H D 2$ Family is Specific to the $H D 2 A$ and $H D 2 D$ Members

To determine the specificity of the glucose response among HD2 members, we quantified the relative levels of $H D 2$ mRNA on induction by soluble carbohydrate. Real-time PCR was carried out in the wild-type Columbia-0 background. Relative to the water control, $H D 2 A$ and $H D 2 D$ mRNA had the most obvious changes in expression level compared to $H D 2 B$ and $H D 2 C$ upon glucose, fructose and sucrose treatment. Furthermore, expression of $H D 2 A$ and $H D 2 D$ in the presence glucose-6-phosphate, fructose-6-phosphate and 3-O-methyl-D-glucose was similar to that of the water control indicating specificity among the sugars. $H D 2 B$ and $H D 2 C$ mRNA levels did not show a significant induction by glucose, fructose or sucrose (Figure 6). In some experiments $H D 2 B$ and $H D 2 C$ mRNA levels appeared to be elevated in the presence of glucose, fructose, and sucrose 
but the levels were within the range of variability of the controls (data not shown).

The results indicate differences among the HD2 family members in responsiveness to sugars. The HD2 family can be sub-divided into two groups based on the sugar inducibility; the sugar-responsive group, HD2A and $H D 2 D$, which appears to be co-ordinately regulated; and the marginally responsive group, $H D 2 B$ and $H D 2 C$.

The induction of $H D 2 A$ and $H D 2 D$ by glucose, fructose or sucrose, which are direct or indirect substrates for hexokinase respectively, and not by 3-O-methyl-D-glucose which is not a substrate for hexokinase, suggest that induction could be mediated through HXK1-dependent signaling. 


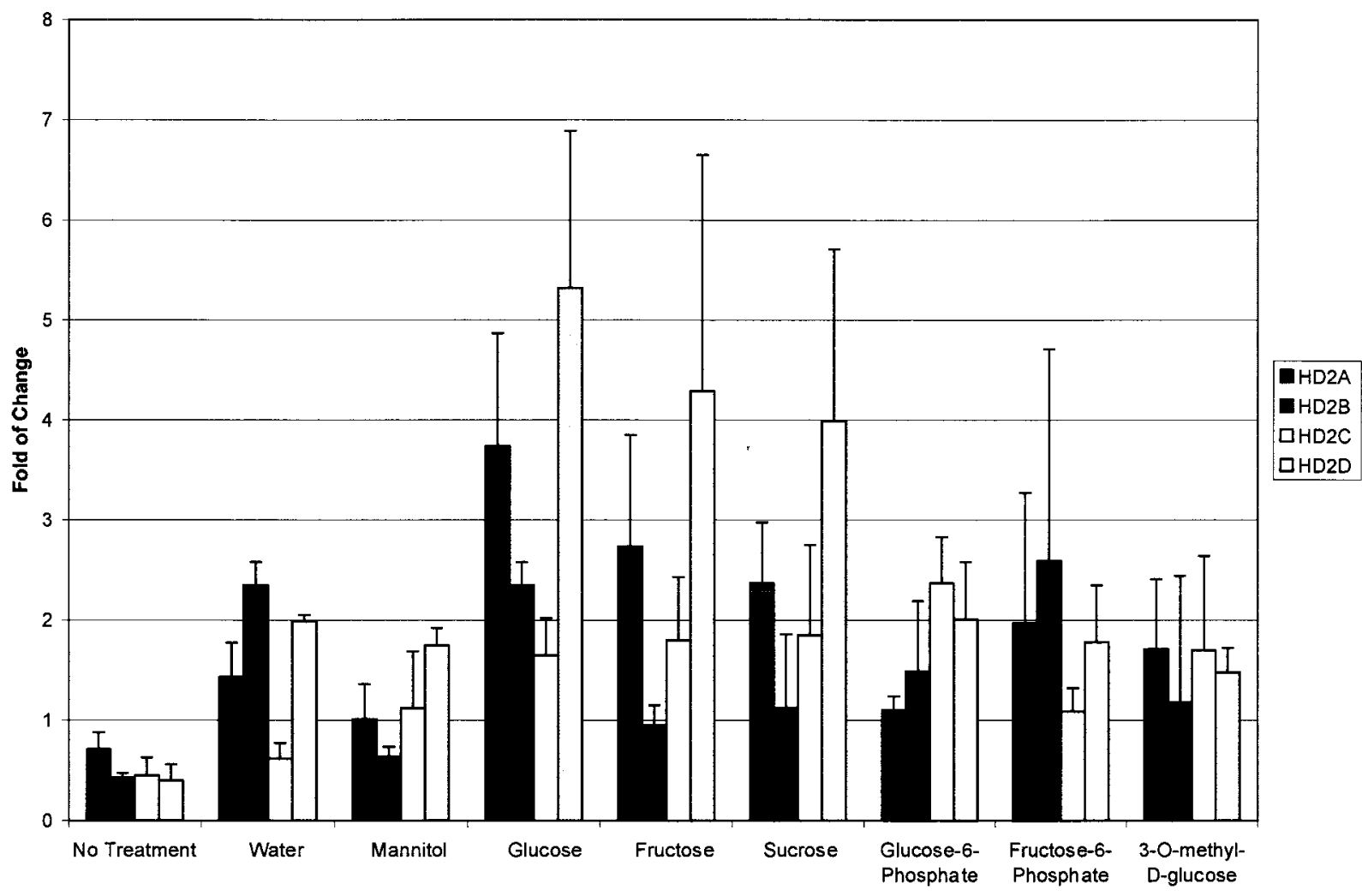

Figure 6. Quantification of soluble carbohydrate induction of the HD2 family $(H D 2 A, H D 2 B, H D 2 C$, and $H D 2 D)$ transcripts by Real-Time PCR in Columbia0 . Values are the mean of three replicate measurements on single RNA samples. Error bars represent the standard deviation above the calculated mean expression value. 
3.3 HD2 Induction by Glucose is Mediated by a Hexokinase-independent Pathway

Hexokinase1 (AtHXK1, gin2 allele) acts as a glucose sensor that integrates light, nutrient, and hormone signaling to control plant growth and development (Jang and Sheen, 1994; Jang et al., 1997; Moore and Sheen, 1999). Since $H D 2 A$ and $H D 2 D$ genes were found to be induced by $H X K$ substrates, it was hypothesized that their expression could be mediated by the HXK1 signal transduction pathway. To test this hypothesis, the AtHXK1 mutant in signaling function (gin2) was obtained from Jen Sheen and further analyses were performed. The phenotype of the gin2-1 null mutant was first confirmed by growing gin2-1 seeds and wild-type Ler (control) seeds on $6 \%$ glucose (330 mM) media. As shown in Figure 7, the gin2-1 seeds were able to germinate and develop cotyledons and true leaves on $6 \%(\mathrm{w} / \mathrm{v})$ glucose (330 mM), while wild-type (Ler) cotyledon and true leaf development were arrested at $6 \%(\mathrm{w} / \mathrm{v})$ glucose $(330 \mathrm{mM})$.

RT-PCR analysis was performed to examine the induction of the HD2 genes in the absence of HXK1 sensing. gin2-1 seeds were germinated in the absence of sucrose and presence of light for 24 hour. Plants were then covered with tin foil to prevent exposure to light. After 4 days, a $3 \%$ glucose (166 mM) solution was added to the plate and total RNA was harvested after 4 hours of exposure in the absence of light. As shown in Figure 8, the HD2 genes in the gin2-1 null mutant behave the same as in the wild-type Ler ecotype. Selective induction of $H D 2 A$ and $H D 2 D$ still occurred in the absence 
of AtHXK1 sensing. Similar results were observed when gin2-1 plants were treated with fructose and sucrose (Figure 9). In this experiment, $H D 2 B$ and $H D 2 C$ may have also been induced but to a lesser extent (Figure 9). The data suggest that the HD2 family may not be a target family in the AtHXK1dependent pathway and may be regulated through a HXK1-independent signaling pathway. This study also supports the previous study in which sugar induction was found to be more persistent for the $H D 2 A$ and $H D 2 D$ members of the $H D 2$ family. 


\section{$6 \%(w / v)$ Glucose}

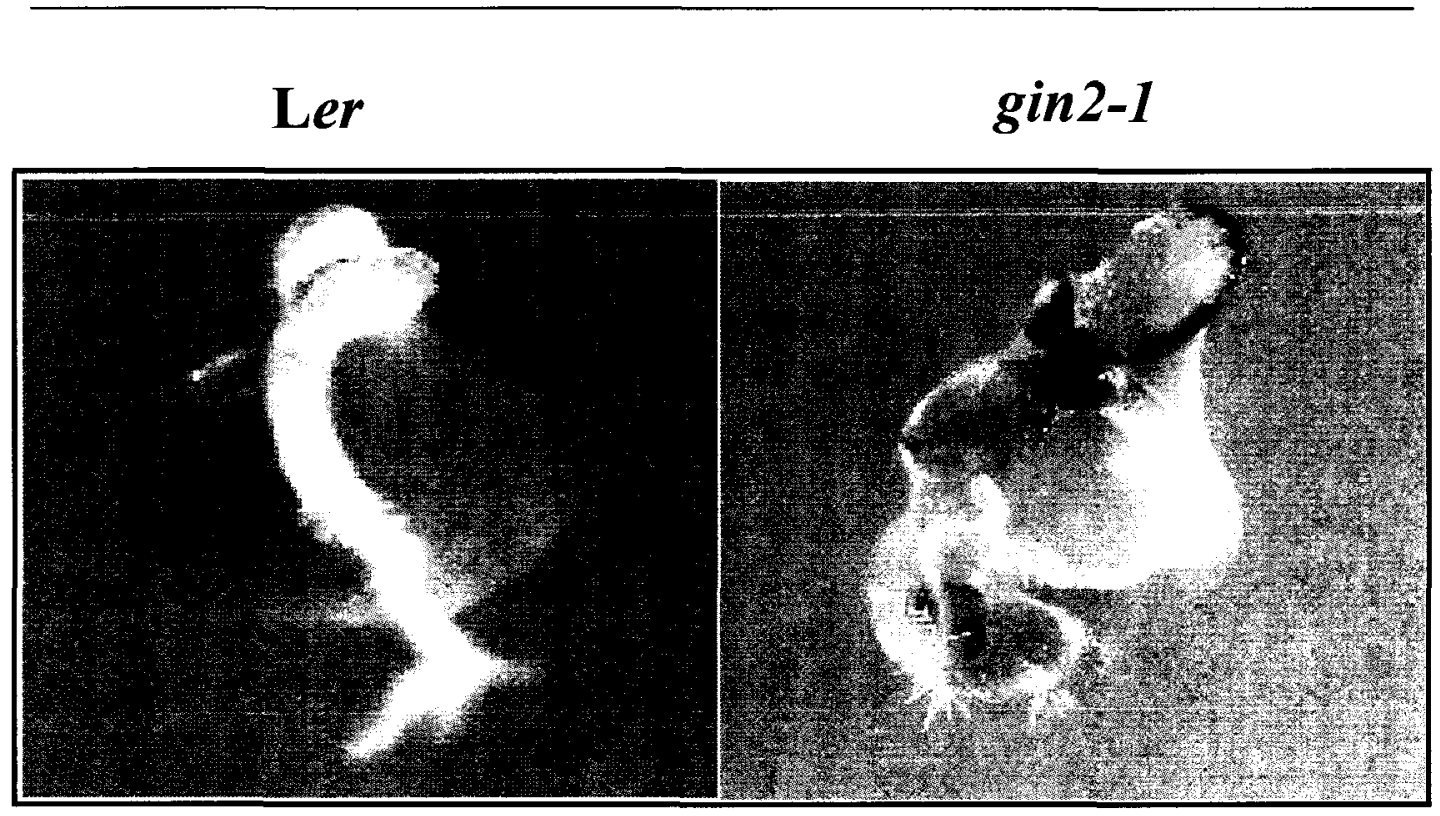

Figure 7. Glucose insensitive-2 (gin2-1) phenotype. Glucose insensitive-2 (gin2-1) and wild-type (Ler) seeds were germinated and grown on $1 / 2$ MS media containing $6 \%(\mathrm{w} / \mathrm{v})$ glucose for one week under continuous light. gin21 mutant seedling was able to develop cotyledon and true leaves under $6 \%$ $(w / v)$ glucose, while wild-type (Ler) seedling cotyledon and true leaves development was arrested at $6 \%(\mathrm{w} / \mathrm{v})$ glucose. 


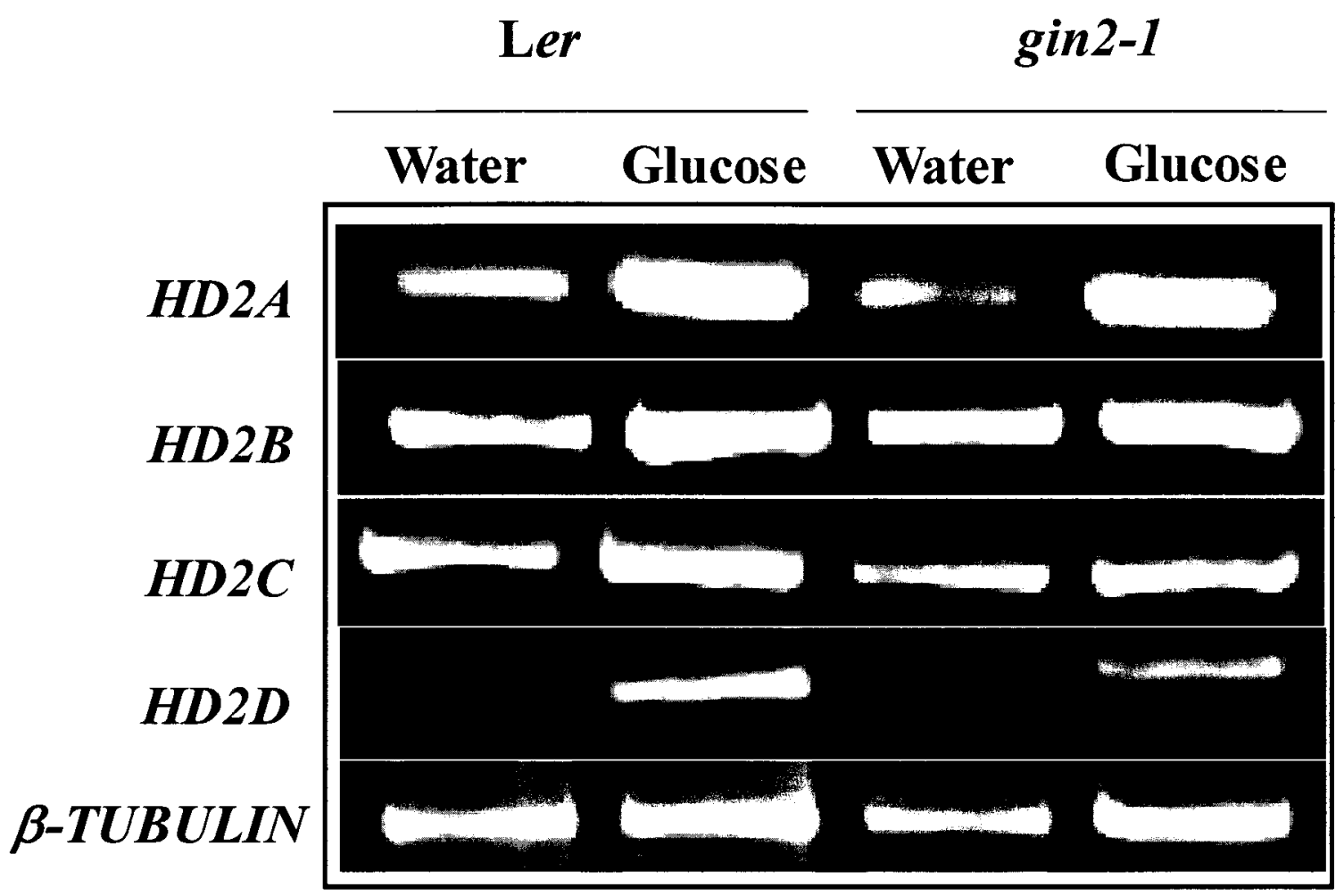

Figure 8. RT-PCR analysis of $H D 2$ induction by $3 \%(\mathrm{w} / \mathrm{v})$ glucose solution in Ler (wt) and gin2-1 null mutant. Plants were germinated in the light on $1 / 2 \mathrm{MS}$, $0.8 \%$ phytagar for 24 hours after 48 hours of stratification at $4^{\circ}$ Celsius. Plants were then incubated in the dark for four days. After four days, plates were sprayed with solutions of $\mathrm{H}_{2} \mathrm{O}$ and $3 \%(\mathrm{w} / \mathrm{v})$ glucose solutions. RNA was extracted after 4 hours of treatment. $2 \mu \mathrm{g}$ of total RNA was converted into CDNA and used as a template for 25 cycles of PCR amplification. The control of this experiment was $\mathrm{H}_{2} \mathrm{O}$. The level of $\mathrm{HD} 2$ genes induction in the gin2-1 null mutant was compared to the level of HD2 genes induction in wild-type Ler. The level of induction in the gin2-1 null mutant appear to be identical to that of the wild-type Ler, in which all HD2 genes (HD2A, HD2B, HD2C, and HD2D) expression were highly induced upon $3 \%(\mathrm{w} / \mathrm{v})$ glucose treatment. $\beta$ TUBULIN was used as a positive internal control for PCR efficiency. 
A)

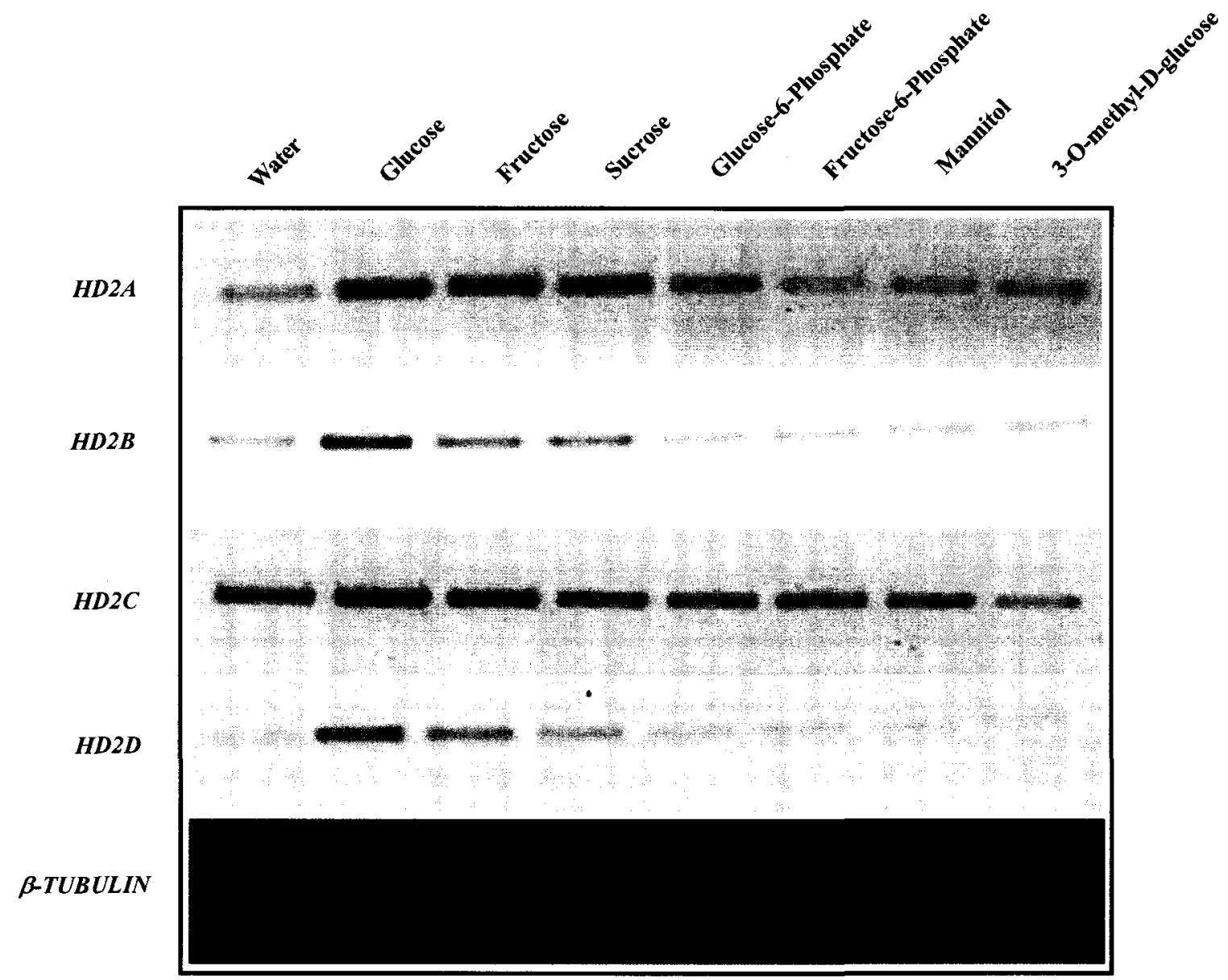

Figure 9. A) RT-PCR analysis of soluble sugars in gin2-1 null mutant. Plants were germinated in the light on $1 / 2$ MS, $0.8 \%$ phytagar for 24 hours after 48 hours of stratification at $4^{\circ}$ Celsius. Plants were then incubated in the dark for four days. After four days, plates were sprayed with solutions of $\mathrm{H}_{2} \mathrm{O}$ and $3 \%$ $(\mathrm{w} / \mathrm{v})$ soluble sugar solutions. RNA was extracted after 4 hours of treatment. $1 \mu \mathrm{g}$ of total RNA was converted into cDNA and used as a template for 25 cycles of PCR amplification. The controls for this experiment include $\mathrm{H}_{2} \mathrm{O}$ and mannitol. $H D 2$ genes $(H D 2 A, H D 2 B, H D 2 C$ and $H D 2 D)$ were examined by RT-PCR. $\beta$-TUBULIN was used as a positive internal control for PCR efficiency. 
B)

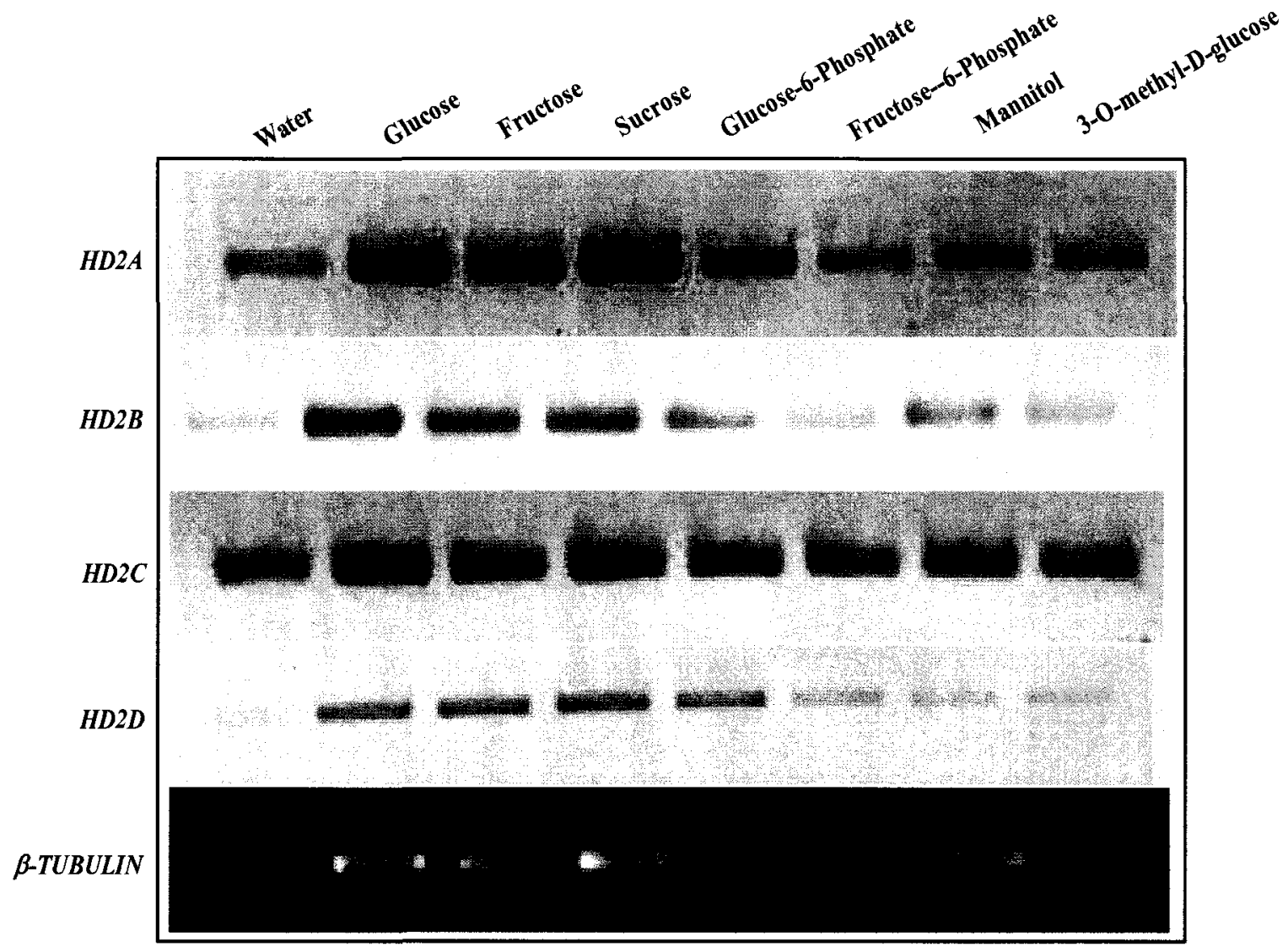

B) RT-PCR analysis of soluble sugars in Ler wild-type control. 


\subsection{HD2 Family Members are Independently Regulated}

Given that $H D 2 A / H D 2 D$ and $H D 2 B / H D 2 C$ behave differently under sugar application, it would be interesting to determine if transcriptional compensation occurs within the HD2 family when one or two of the genes were disrupted. $H D 2 A$ and $H D 2 C$ loss-of-function mutants were previously identified by Colville (2007). Mutants of these two genes are important for this study as there is a mutation in one of the glucose-inducible members and one in the glucose-non inducible members. In addition to these two mutations, a double loss-of-function mutant of $H D 2 A$ and $H D 2 C$ was created by Colville (2007). $H D 2 D$ and $H D 2 B$ mutants were also searched (Materials and Methods, Appendix I) to create a complete knockout of the HD2 family, however, no knockout mutants in $H D 2 B$ or $H D 2 D$ could be found.

To determine whether there is compensation at the transcriptional level upon glucose induction within the HD2 family, single $h d 2 a$ and $h d 2 c$ loss of function mutants, as well as a double $h d 2 a$ hd2c mutant were analyzed. Seeds were germinated in the absence of sucrose and presence of light for 24 hour. Plants were then covered with tin foil. After 4 days, a $3 \%$ glucose (166 mM) solution was added to the plate and total RNA was harvested after 4 hours of exposure in the absence of light. RT-PCR of $H D 2 B$ and $H D 2 D$ transcript was examined for potential transcriptional up-regulation in the absence of the HD2C and HD2A respectively (Figure 10). $\beta$-TUBULIN was used as a positive control to ensure the quality of RNA and the efficiency of the PCR reaction. Figure 10 shows that there was a thin $H D 2 A$ band in the 
hd2a loss-of-function mutant, upon glucose treatment. Since the primers used do not flank the T-DNA insertion site; a small portion of the HD2A gene may be transcribed. $h d 2 c$ is a null mutant with a complete loss of $H D 2 C$ transcription. There was no accumulation of $H D 2 C$ mRNA in the $h d 2 c$ mutant background upon water or glucose treatment. In addition, there was no HD2A or HD2C mRNA accumulation in the double $h d 2 a$ hd2c mutant background. The potential redundant partners of $H D 2 C$ and $H D 2 A(H D 2 B$ and $H D 2 D$, respectively) were transcribed in the mutants and there was no apparent change in response of $H D 2 B$ or $H D 2 D$ mRNA levels in the absence or presence of glucose relative to wild-type. $\beta-T U B U L I N$ was used as a positive control to check the quality of RNA and the efficiency of the PCR reaction. The $\beta$-TUBULIN control of the PCR reaction was elevated in the $h d 2 c$ mutant sample. Although semi-quantitative RT-PCR is not the most accurate method to identify changes in the regulation of gene transcription in the background of mutants lines, it was sufficient here to show that the deletion mutation did not alter the transcription of the other HD2 genes.

$H D 2 A, H D 2 B$, and $H D 2 D$ genes were analyzed for potential transcriptional down-regulation as a compensatory mechanism for constitutive expression of HD2C (35S::HD2C-GFP). RT-PCR analysis (Figure 11) indicated that over-expression of one of the HD2 members (35S::HD2C-GFP) had no effect on the transcript level of the other HD2 members by $3 \%$ glucose (166 mM) treatment. The combined data indicated that the HD2 genes are regulated independently. Potential redundant partners $H D 2 A / H D 2 D$ and 
$H D 2 B / H D 2 C$ did not display altered expression patterns when one of the pattners was silenced by a T-DNA insertion and responded to sugar signals in the same manner as wild-type lines. The three mutant lines therefore provide material to examine the functional consequences of specific HD2 gene loss by phenotypic analyses. 


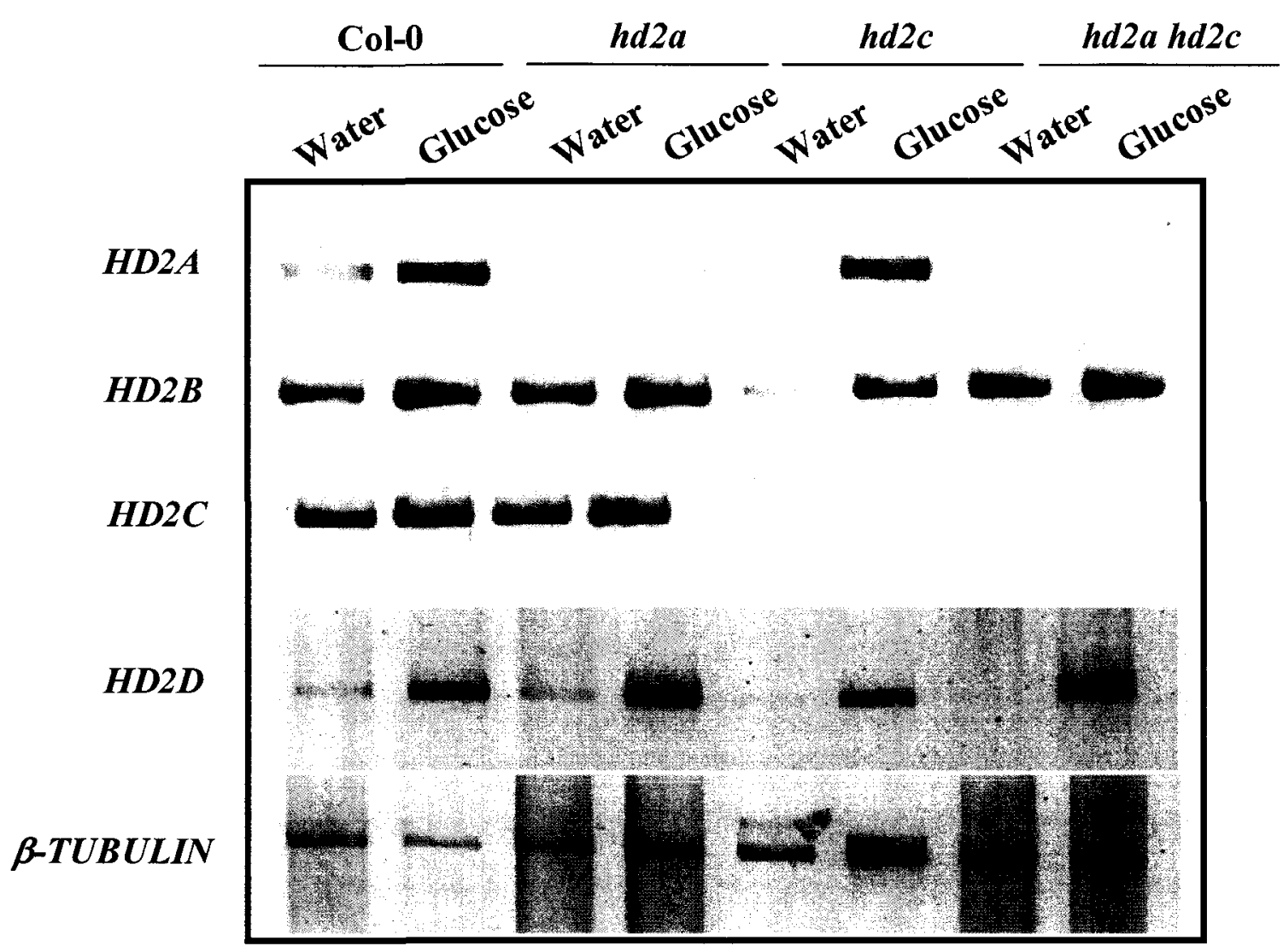

Figure 10. RT-PCR analysis of HD2 mutant lines. RT-PCR analysis was carried out to detect upregulation of other HD2 genes in the absence of one or two functioning $H D 2$ genes upon $3 \%$ glucose. Plants were germinated in the light on $1 / 2 \mathrm{MS}, 0.8 \%$ phytagar for 24 hours after 48 hours of stratification at $4^{\circ}$ Celsius. Plants were then incubated in the dark for four days. After four days, plates were sprayed with solutions of $\mathrm{H}_{2} \mathrm{O}$ and $3 \%(w / v)$ glucose solutions. RNA was extracted after 4 hours of treatment. $2 \mu \mathrm{g}$ of total RNA was converted into cDNA and used as a template for 25 cycles of PCR amplification. The control of this experiment was $\mathrm{H}_{2} \mathrm{O}$. Other members $(H D 2 B$ and $H D 2 D$ ) were induced to a similar level to that of the wild-type control and there was no up regulation of $H D 2 B$ or $H D 2 D$ in the background mutants. $\beta$ TUBULIN was used as a positive internal control for PCR efficiency. 


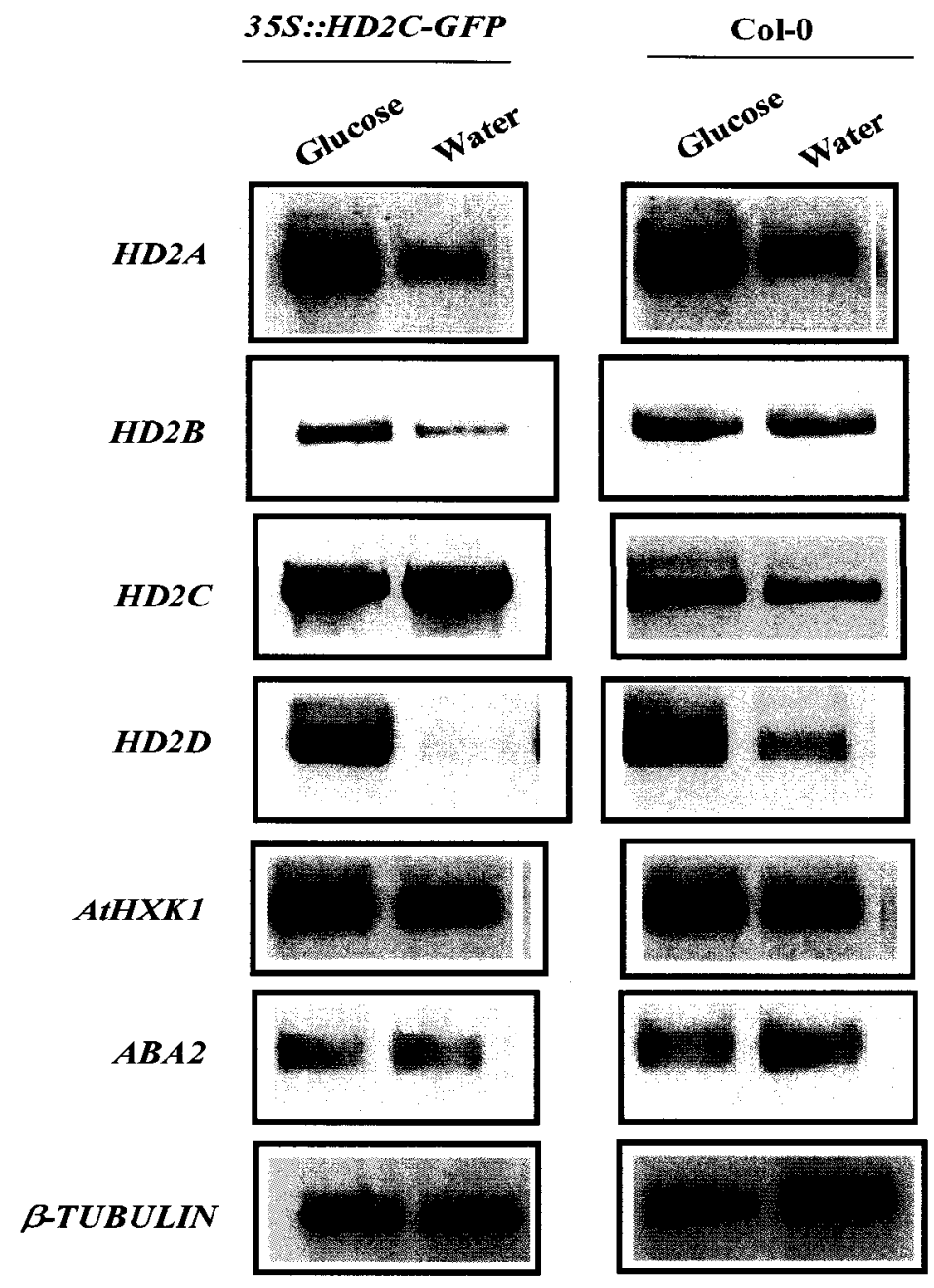

Figure 11 : RT-PCR analysis of $H D 2$ induction by $3 \%(\mathrm{w} / \mathrm{v})$ glucose solution in the 35S::HD2C-GFP and wild-type Col-0 background. Plants were germinated in the light on $1 / 2$ MS, $0.8 \%$ phytagar for 24 hours after 48 hours of stratification at $4^{\circ}$ Celsius. Plants were then incubated in the dark for four days. After four days, plates were sprayed with solutions of $\mathrm{H}_{2} \mathrm{O}$ and $3 \%(\mathrm{w} / \mathrm{v})$ glucose solutions. RNA was extracted after 4 hours of treatment. $2 \mu \mathrm{g}$ of total RNA was converted into CDNA and used as a template for 25 cycles of PCR amplification. The control of this experiment was $\mathrm{H}_{2} \mathrm{O}$. The level of $\mathrm{HD} 2$ genes induction in the 35S::HD2C-GFP background was compared to the level of $H D 2$ genes induction in wild-type Col-0. The level of induction of the other HD2 members in the 35S::HD2C-GFP background appear to be similar to that of the wild-type Col-0, in which all HD2 genes (HD2A, HD2B and HD2D) expression were highly induced upon $3 \%(\mathrm{w} / \mathrm{v})$ glucose treatment, and were not affected by the overexpression of HD2C. $\beta$-TUBULIN was used as a positive internal control for PCR efficiency. 


\subsection{Roles of HD2 Family Members in Seed Germination}

Glucose signaling has been implicated in regulating the transition from quiescent to germinative embryos (Price et al., 2003; Dekkers et al., 2004; Yuan, 2006). Sugars are known to delay seed germination and arrest seedling development. An assay for plant sugar signaling has been developed in which delays in germination and post-germinative development such as cotyledon greening and expansion, hypocotyls elongation, true leaf development, and root growth are assessed (reviewed by Price et al., 2003).

Previous research (Wu et al., 2000b) showed the involvement of HD2A in embryo development and since $H D 2 A / H D 2 D$ genes are also induced by sugar in post-germinative seedlings we wanted to investigate their role in germination. Thus, we used HD2 null mutants and over-expression lines to correlate composition of the HD2 family and germination. Our analysis revealed that the HD2A-GFP over-expression line (35S::HD2A-GFP) and hd2a null mutant both exhibited accelerated germination relative to wt controls, $h d 2 c$ mutants and $h d 2 a h d 2 c$ mutants (Figure 12). In contrast $h d 2 c$ mutants exhibited a slower germination rate that took up to almost 72 hours for full germination to occur. This data provided evidence that HD2A and $H D 2 C$ may play different roles in seed germination. Furthermore, it appeared that $H D 2 A-G F P$ yielded the same response as the $h d 2 a$ null mutant suggesting that $H D 2 A-G F P$ is acting functionally as a competitive inhibitor in the $35 S:: H D 2 A-G F P$ lines. 
In addition, we also studied the effect of glucose on germinative and post-germinative development of $H D 2$ null mutants and over-expression lines. Our data showed that glucose-induced delay in germination is enhanced in hd2c null mutants and deminished in hd2a null mutants and the HD2A-GFP transgenic plants line (Figure 13A). hd2a hd2c double mutants were more resistance to glucose than $h d 2 c$ null mutants but to a lesser extent than $h d 2 a$ null mutants confirming the different and opposing roles of $H D 2 A$ and $H D 2 C$ in germination. Post-germinative development was assayed by measuring root length at different concentration of glucose as described in Sridha and Wu (2006). This measurement did not account for the differences in secondary root proliferation. All of the mutants and transgenic lines displayed a reduction in root length as we increased the glucose concentration (Figure 13B). This data supports the previous germination data and shows that $H D 2 A$ and $H D 2 C$ have different and opposite functions in germination and a less significant role in post-germinative development. 


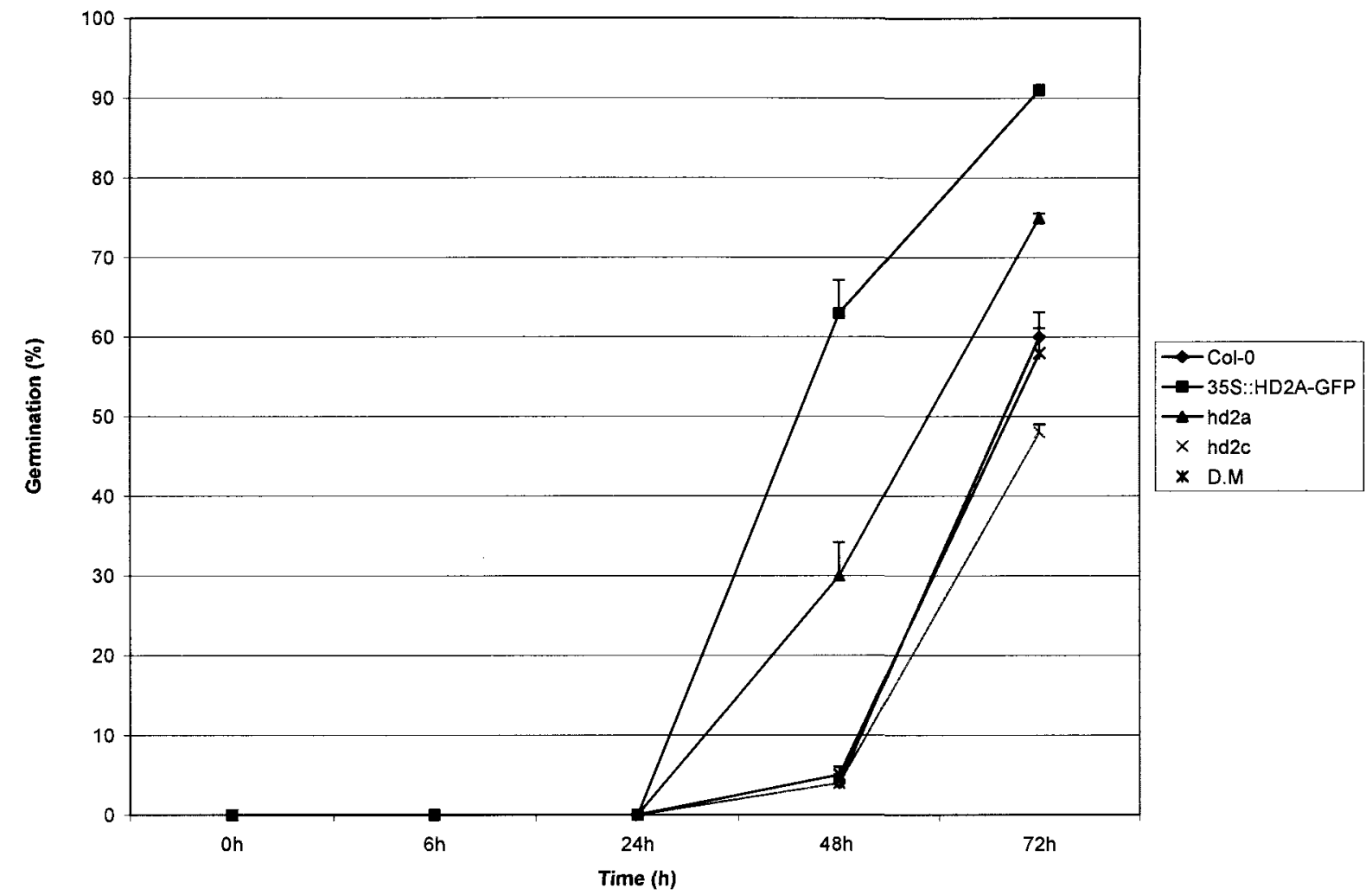

Figure 12. Seed germination analysis. Germination rate analysis of 35S::HD2A-GFP, hd2a, $h d 2 c$, and $h d 2 a$ hd2c (double mutants (D.M)) seeds on MS medium. Germination (fully emerged radicle) was scored at various time points. Values are average of 3 biological replicates $(n=3)$. Error bars represent standard deviation. 
A)

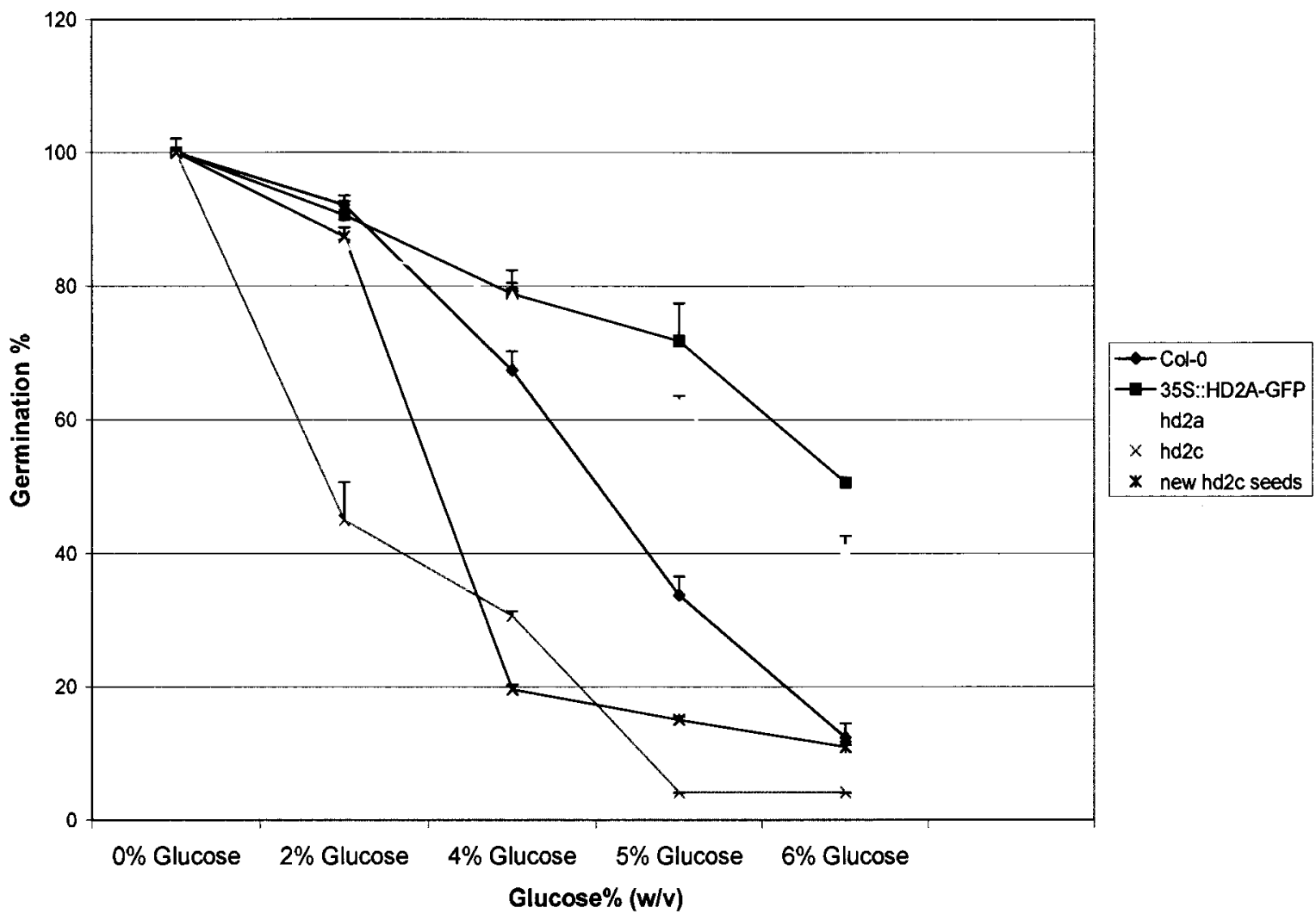

Figure 13: Glucose effect on seed germination (A) and root length (B). Germination and root analysis of 35S::HD2A-GFP, hd2a, hd2c, hd2a hd2c (double mutants (D.M)) seeds under different concentrations of glucose. (A) Number of seedlings was scored 4 days after germination. (B) Root length was measured 6 days after germination. Values are average of 3 biological replicates $(n=3)$. Error bars represent standard deviation. 
B)

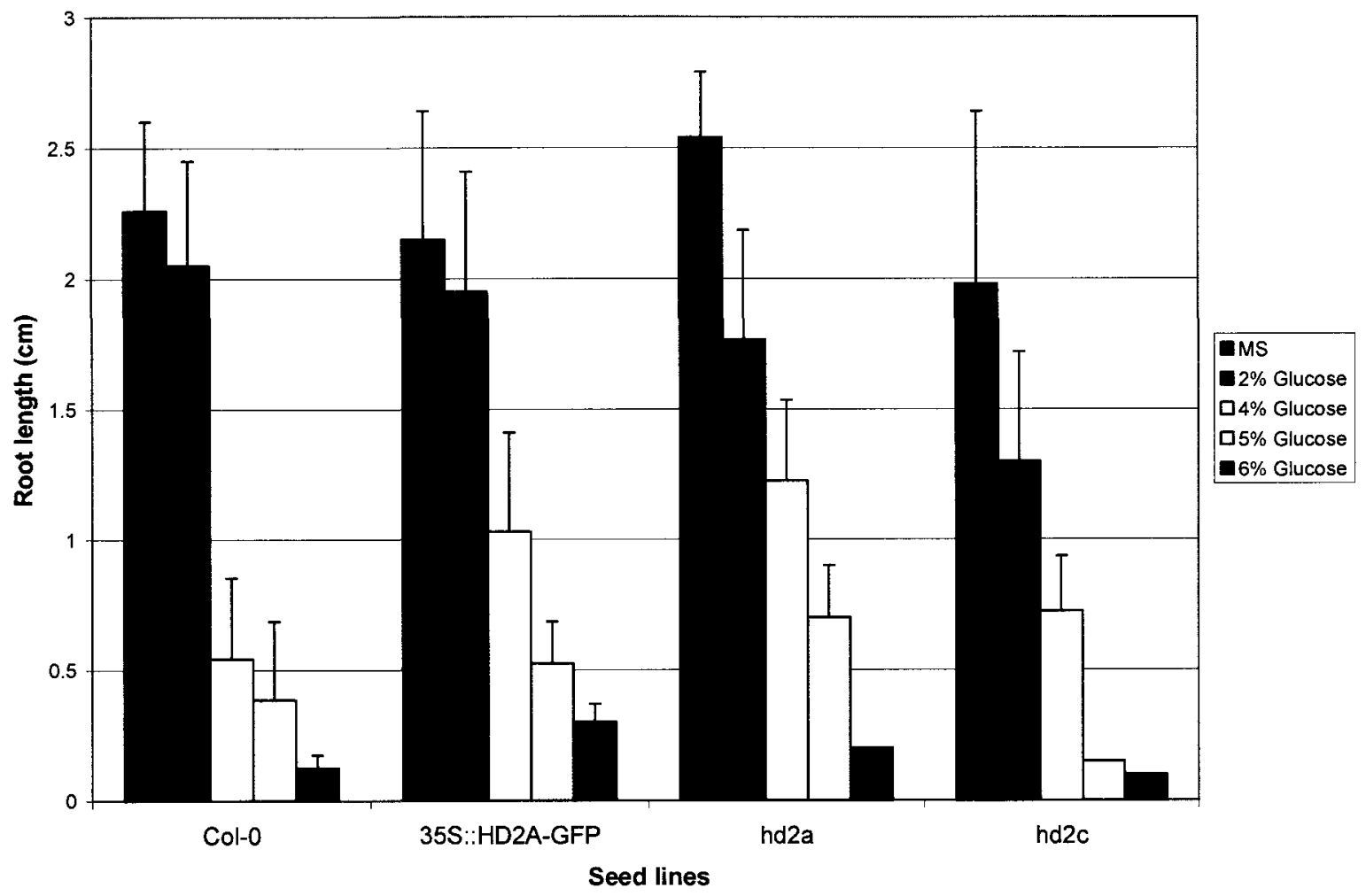




\subsection{HD2 Function in Post-germinative Development}

Many studies have linked different Abscisic acid (ABA) signaling pathways downstream of the glucose signaling pathway (Yamagishi et al., 2009). Microarray studies have confirmed that the expression of a variety of genes associated with carbohydrate metabolism, signal transduction, and metabolite transport are co-regulated by sugar and ABA (Price et al., 2004, Li et al., 2006). The phytohormone ABA has been shown to regulate aspects of plant growth and development such as germination, seedling growth, seed development, seed dormancy, the transition from vegetative to reproductive phase as well as the responses to environmental stress including drought, cold, and salt (Finkelstein et al., 2002).

Recent findings show that epigenetic regulation plays a role in ABAmediated plant processes and links $A B A$ signaling to chromatin remodeling (reviewed by Chinnusamy et al., 2008). The finding that $H D 2 A$ is required for H3 Lys9 deacetylation and subsequent H3 Lys9 methylation verify that HD2 proteins modulate gene expression through histone modification (Lawrence et al., 2004). Other studies have shown that histone acetylation is involved in response to abiotic stresses (Kim et al., 2004; Lee et al., 2005; Song et al., 2005; Stockinger et al., 2001; Vlachonasios et al., 2003). Stockinger et al., (2001) proposed that histone acetyltransferases may be employed through transcription factors, such as the AP2/ERF transcription factor, CBF1, to regulate cold-induced genes. Song et al., (2005) found that AtERF7 (the transcription repressor) can interact with the HDAC complexes to repress 
ABA induced and stress response genes. These findings reveal that gene expression in the plant response to abiotic stresses may be chromatin controlled via the acetylation and deacetylation of histones on target genes. Sridha and Wu (2006) and Fu et al., (2007) found that a number of the HDACs members were repressed by ABA in Arabidopsis thaliana and rice. Sridha et al., (2006) showed that $A t H D 2 C$ is repressed by abscisic acid and in over-expression of AtHD2C-GFP transgenic plants conferred an $A B A$ insensitive phenotype. Furthermore, the expressions of several abscisic acidresponsive genes were found to be effected in the 35S::AtHD2C-GFP plants suggesting that $A t H D 2 C$ may modulate abscisic acid.

\subsubsection{Modulation of HD2 Expression by the Plant Hormone ABA}

We considered the possibility that $H D 2 A / H D 2 D$ induction through glucose signaling during germination results in chromatin remodeling and induction of $A B A$ genes which repress $H D 2 C / H D 2 B$ genes during postgerminative development. As shown in Figure 14 our results show $H D 2 A / H D 2 D$ and $H D 2 C / H D 2 B$ are repressed by $100 \mu \mathrm{m}$ ABA. Furthermore, ABA biosynthesis gene expression (ABA2, AAO3) over 16 hours of glucose treatment did not correlate with HD2 induction by glucose. HD2 induction initiated after 4 hours of glucose treatment and began to decline after 16 hours of glucose treatment (Figure 15); whereas, $A B A 2$ and $A A O 3$ gene expression was constant during the same period of time. This data differed from the previous report from Jen Sheen's lab (Cheng et al., 2002). They allowed 
seeds to germinate under normal growth conditions for two weeks with the presence of glucose in the MS media; whereas, in this analysis tissues were starved of sugar for 4 days before glucose reintroduction. Both seedling age and methodology could account for the different results. In addition, a different study by Price et al., 2003 indicated that the earliest induction of ABA2 upon glucose treatment was noticed two days after germination, and that the induction rate correlated with the onset of germination and not with glucose concentration indicating that glucose may not increase $A B A$ biosynthesis during germination by transcriptional up-regulation. This was consistent with our results and suggests that the glucose induction of $H D 2 A / H D 2 D$ is not directly linked to the ABA pathway at the transcriptional level. 


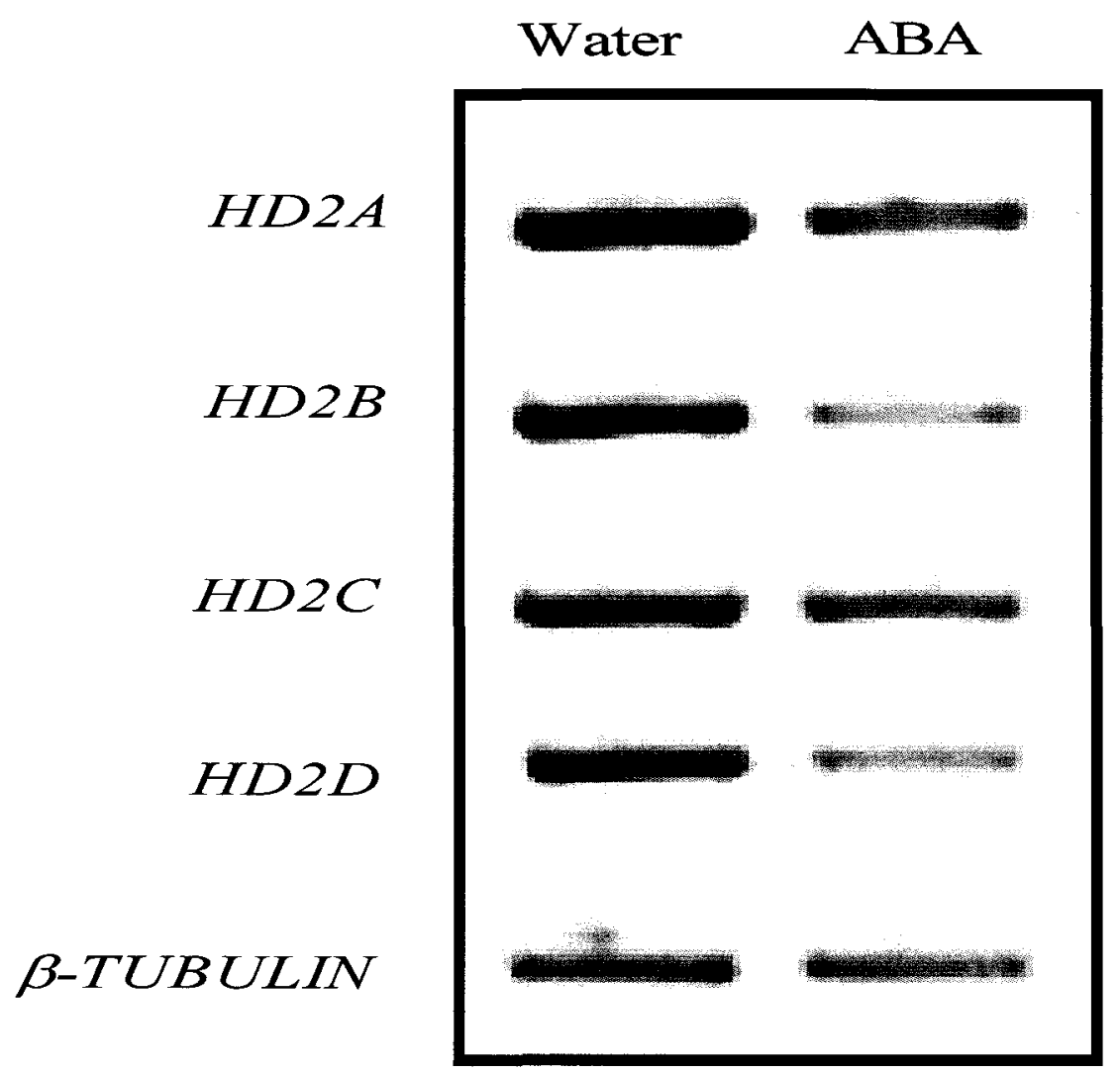

Figure 14. RT-PCR analysis of $H D 2$ repression by $100 \mu \mathrm{m}$ ABA. Plants were germinated in the light on $1 / 2 \mathrm{MS}, 0.7 \%$ phytagar for 10 days after 48 hours of stratification ( $4^{\circ}$ Celsius). Plants were then treated with solution of water or $100 \mu \mathrm{m} A B A$ and incubated in the growth chamber for 6 hours. RNA was then extracted and $2 \mu \mathrm{g}$ of total RNA was converted to cDNA which served as a template for 25 cycles of PCR amplification. Water served as a control. All HD2 genes were repressed upon $100 \mu \mathrm{m}$ ABA. $\beta-T U B U L I N$ was used as a positive control. 


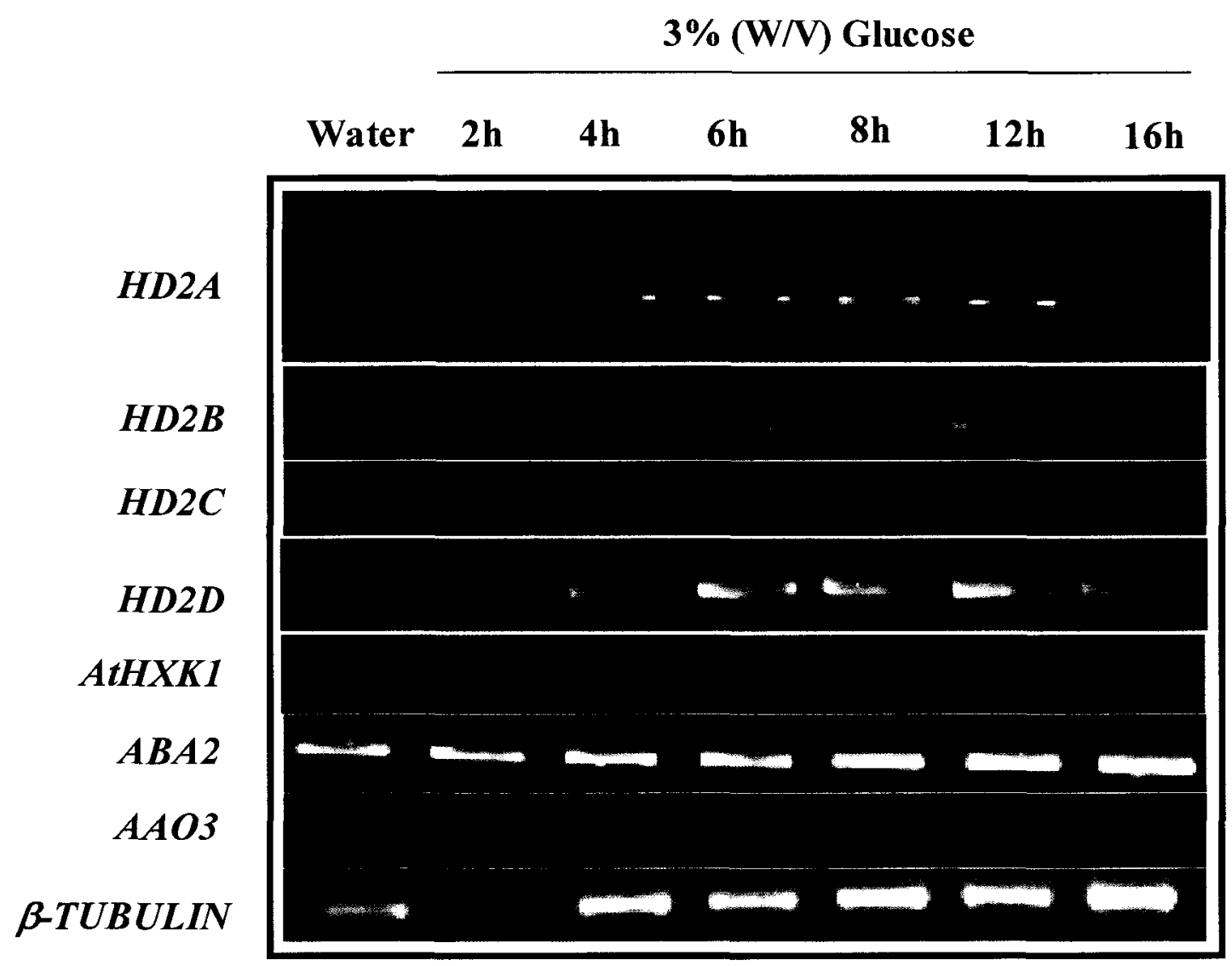

Figure 15. RT-PCR analysis of $H D 2$ and ABA biosynthesis gene expression over 16 hours in response to $3 \%(\mathrm{w} / \mathrm{v})$ glucose application. Wild-type Col-0 seeds were germinated in the light on $1 / 2$ MS, $0.8 \%$ phytoagar for 24 hours after 48 hours of stratification at $4^{\circ}$ Celsius. Plants were then incubated in the dark for four days. After four days, plates were sprayed with solutions of $\mathrm{H}_{2} \mathrm{O}$ and $3 \%(\mathrm{w} / \mathrm{v})$ glucose solutions. RNA was extracted after $4 \mathrm{~h}, 6 \mathrm{~h}, 12 \mathrm{~h}, 14 \mathrm{~h}$ and $16 \mathrm{~h}$ of treatment. $2 \mu \mathrm{g}$ of total RNA was converted into cDNA and used as a template for 25 cycles of PCR amplification. HD2 induction initiates after 4hours of glucose treatment and remains constant up to 16 hours of glucose treatment, while the expression of ABA biosynthesis genes remains unchanged. $\beta$-TUBULIN was used as a positive internal control for PCR efficiency. 


\subsubsection{Relationship of glucose/HD2 and ABA Signal Transduction Pathways}

Since $A B A$ is known to be involved in chromatin remodeling, we wanted to determine if a functional relationship existed between specific deacetylases such as AtHD2A or AtHD2C, and ABA, abiotic stress. Wild-type seeds exhibited enhanced delays in germination and development at $0.2 \mu \mathrm{m}$ ABA while $35 S:: H D 2 A-G F P$ seeds displayed decreased sensitivity to $A B A$ and were able to germinate and develop healthy cotyledons (Figure 16A) and did not show true leaves at $0.2 \mu \mathrm{m}$ ABA. A similar phenotype, but to a lesser extent was also observed by the $h d 2 a$ mutants. The $h d 2 c$ mutant seeds were extremely sensitive to $0.2 \mu \mathrm{m} A B A$ and exhibited similar phenotype to that of wild-type. $h d 2 a h d 2 c$ double mutants exhibited sensitivity to $0.2 \mu \mathrm{m} A B A$ (Figure 16A). Furthermore, as shown in Figure 10B, $0.2 \mu \mathrm{m}$ ABA caused a $66 \%$ decrease in the germination of the wild-type seeds, $35 \%$ decrease in the rate of the $35 S:: H D 2 A-G F P, 47 \%$ decrease in the germination rate of $h d 2 a$, $89 \%$ decrease in the germination rate of $h d 2 c$, and $50 \%$ decrease in the germination rate of double mutants (D.M). In addition, as shown in Figure 17 $A \& B, 0.1 \mu \mathrm{m}$ and $0.2 \mu \mathrm{m}$ ABA reduced root length in all wild-type, mutants and over-expression line. Our result suggested that exogenous $A B A$ acts to delay germination and post-germinative development of roots in all of the mutant and transgenic lines but accentuates their differences as described earlier. Effectively, mutation and possibly inhibition of HD2A expression by HD2A-GFP did not offset the dramatic impact of ABA on post germinative root development. Mutation of $H D 2 C$ expression resulted in greater delays in 
germination and post-germinative development in the presence of ABA. It therefore appears that the functional differences attributable to HD2A and $H D 2 C$ during germination are in effect upstream of the phenotypic effects induced by exogenous $\mathrm{ABA}$ during post-germinative development. As $H D 2 A$ is responsive to glucose this may position the glucose/HD2A pathway upstream of the ABA pathway. 
Figure 16. Phenotypic analysis of 35S::HD2A-GFP, hd2a, hd2c, and $h d 2 a$ $h d 2 c$ (double mutants (D.M)) under stress conditions $(0.1 \mu \mathrm{m}$ and $0.2 \mu \mathrm{m}$ ABA). Col-0 wild-type, 35S::HD2A-GFP, hd2a, hd2c, and hd2a hd2c seeds were stratified for 48 hours at $4^{\circ} \mathrm{C}$ Celsius and sown on $1 / 2 \mathrm{MS}, 0.7 \%$ phytagar minimal media supplemented with 0 (MS media), $0.1 \mu \mathrm{m}$ and $0.2 \mu \mathrm{m}$ in the growth incubator under continues florescence light (24 hour). Col-0 wild-type was used as a control for this study.

A) Seedlings were observed under the WDS 54 Nikon JAPAN microscope after 4 days of germination. Wild-type seeds exhibited growth arrest and further development inhibition at $0.2 \mu \mathrm{m}$ ABA while $35 \mathrm{~S}:: H D 2 A-$ GFP seeds displayed decreased sensitivity to $A B A$ and were able to germinate develop healthy cotyledons and true leaves at $0.2 \mu \mathrm{m}$ ABA. A similar phenotype, but to a lesser extend was also observed by the hd2a mutants. The $h d 2 c$ mutant seeds were extremely sensitive to 0.2 $\mu \mathrm{m} A B A$ and exhibited similar phenotype to that of wild-type. hd2a $h d 2 c$ double mutants exhibited a moderate sensitivity to $0.2 \mu \mathrm{m} \mathrm{ABA}$, less than wild-type and $h d 2 c$ mutants.

B) Germination rate analysis. Effect of $A B A$ on germination rate was scored 4 days after germination. $0.2 \mu \mathrm{m}$ ABA caused a $66 \%$ decrease in the germination rate of the wild-type seeds, $35 \%$ decrease in the rate of the 35S::HD2A-GFP, $47 \%$ decrease in the germination rate of hd2a, $89 \%$ decrease in the germination rate of $h d 2 c$, and $50 \%$ decrease in the germination rate of double mutants (D.M). Values are average of 3 replicates $(n=3)$. Error bars represent standard deviation. 
A)

MS Media
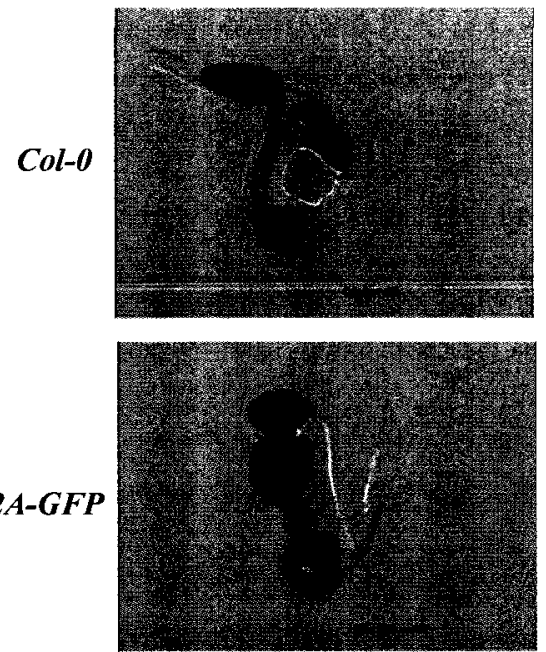

hd $2 a$ mutant

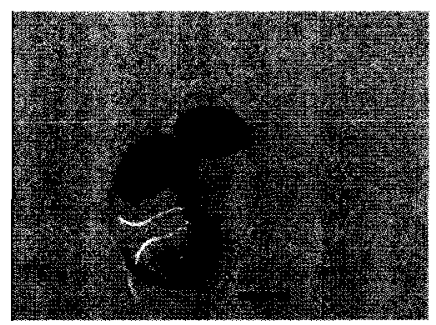

$h d 2 c$ mutant

$h d 2 a h d 2 c$

double mutant
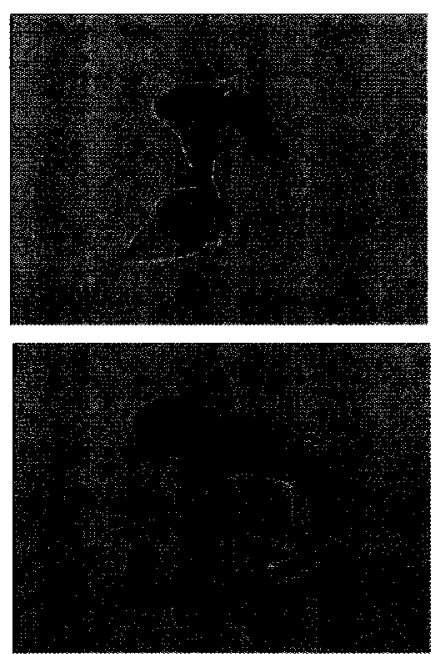

$0.1 \mu \mathrm{m}$ ABA
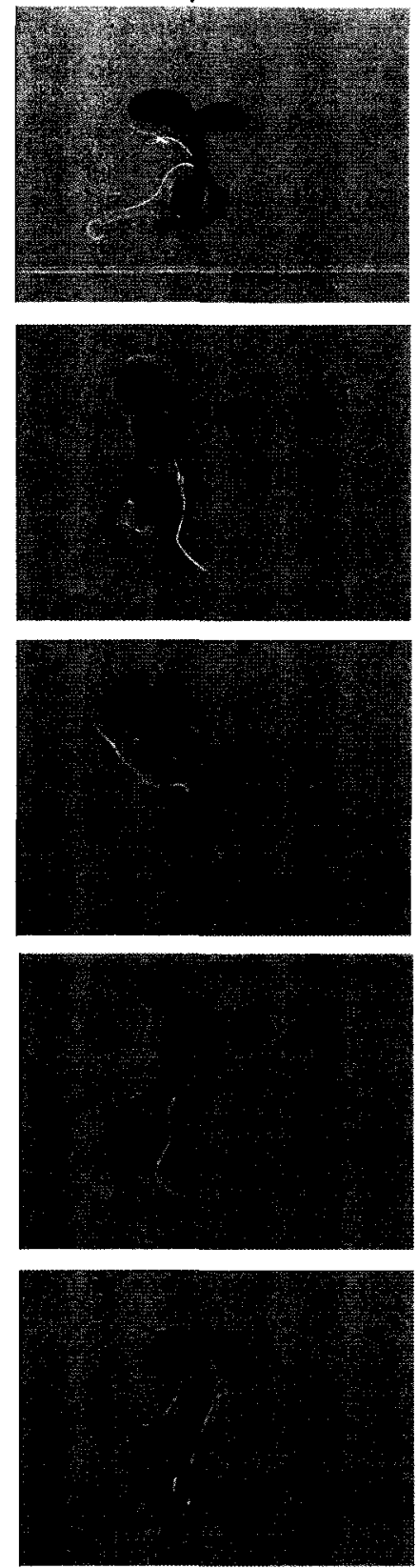

$0.2 \mu \mathrm{m}$ ABA
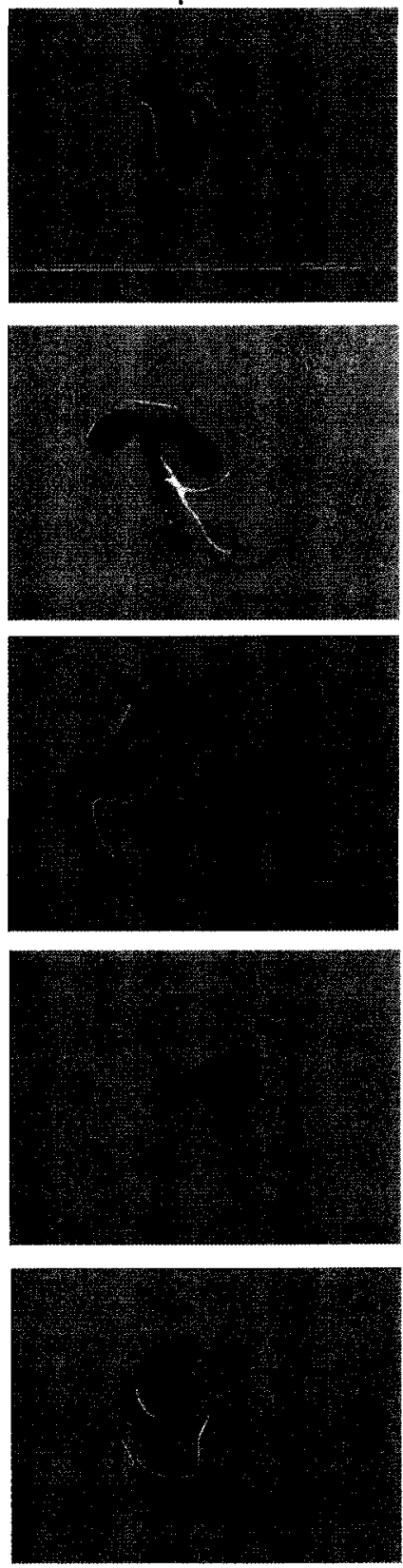
B)

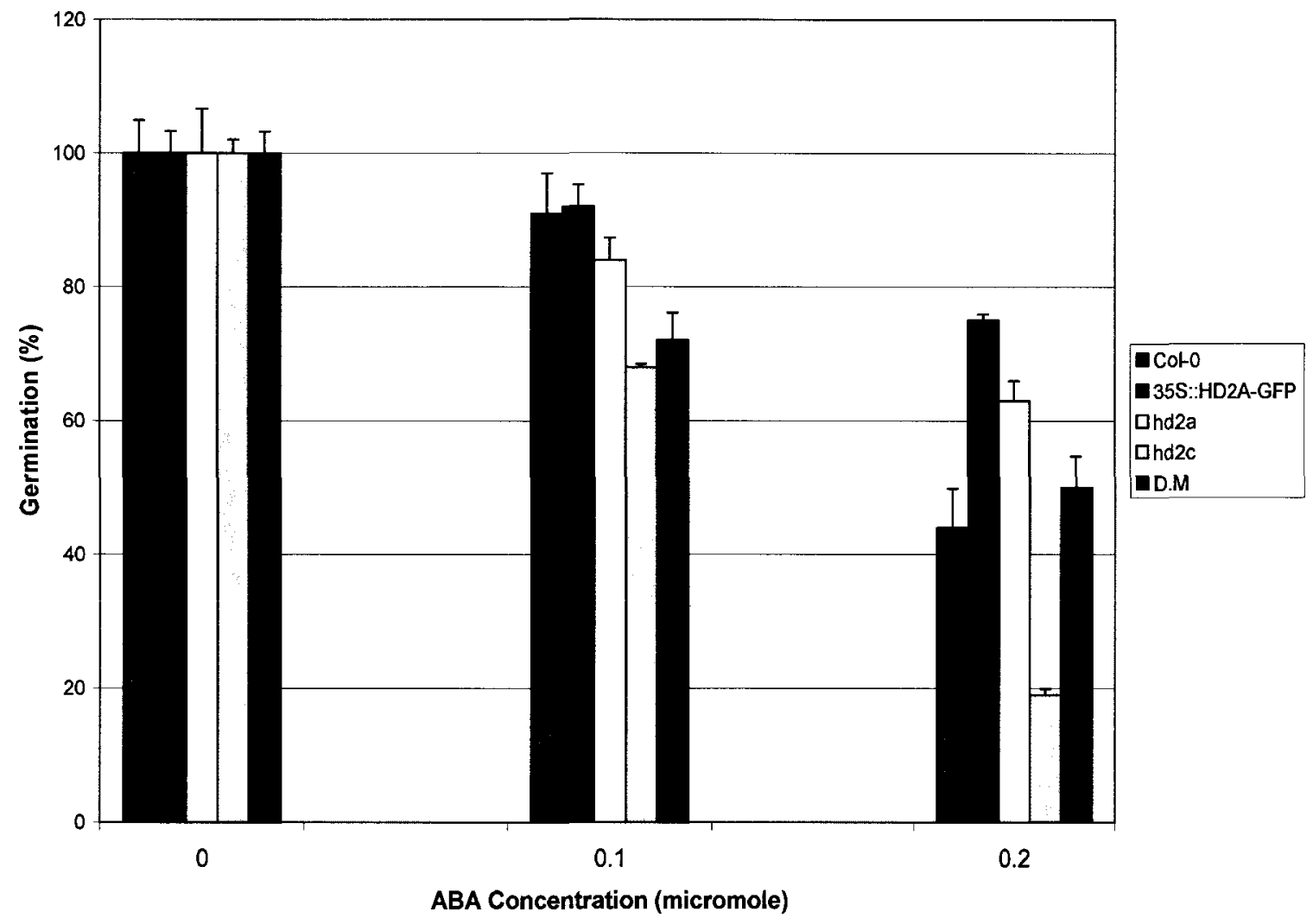


Figure 17. Root phenotypic analysis of 35S::HD2A-GFP, hd2a, hd2c, and hd2a hd2c (double mutants (D.M)) under osmotic stress conditions $(0.1 \mu \mathrm{M}$ $A B A$ and $0.2 \mu \mathrm{M} A B A$ ). Col-0 wild-type was used as control for this study. Seeds were germinated on a minimal media supplemented with 0 (MS media), $0.1 \mu \mathrm{M}$ ABA and $0.2 \mu \mathrm{M}$ ABA. Root length was then observed after 5 days of germination.

A) All seeds showed a reduction in root length under $0.2 \mu \mathrm{M} A B A$.

B) Root length measurements analysis. Values are average of 3 replicates $(n=3)$ and error bars represent standard deviation. 
A)

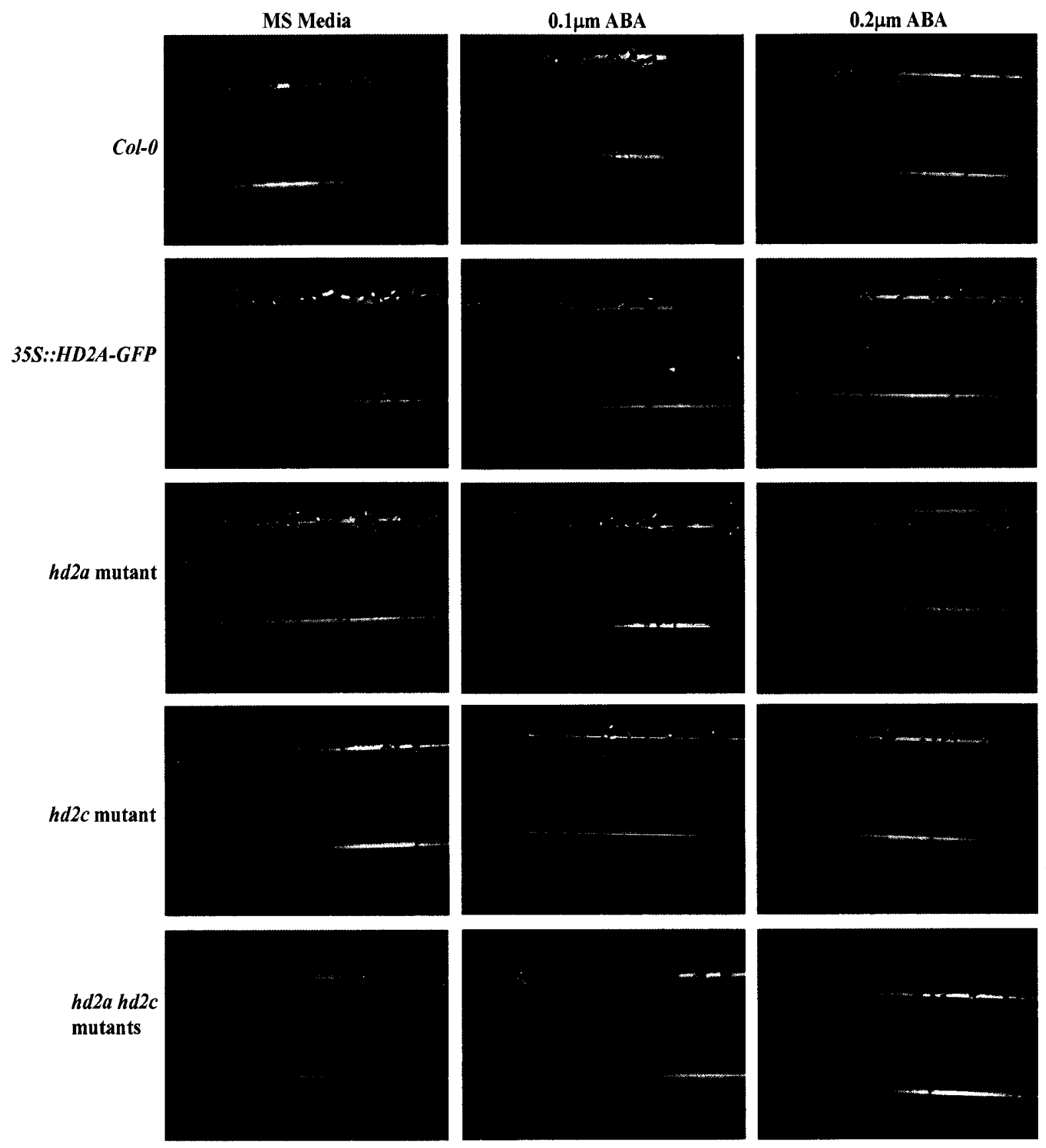


B)

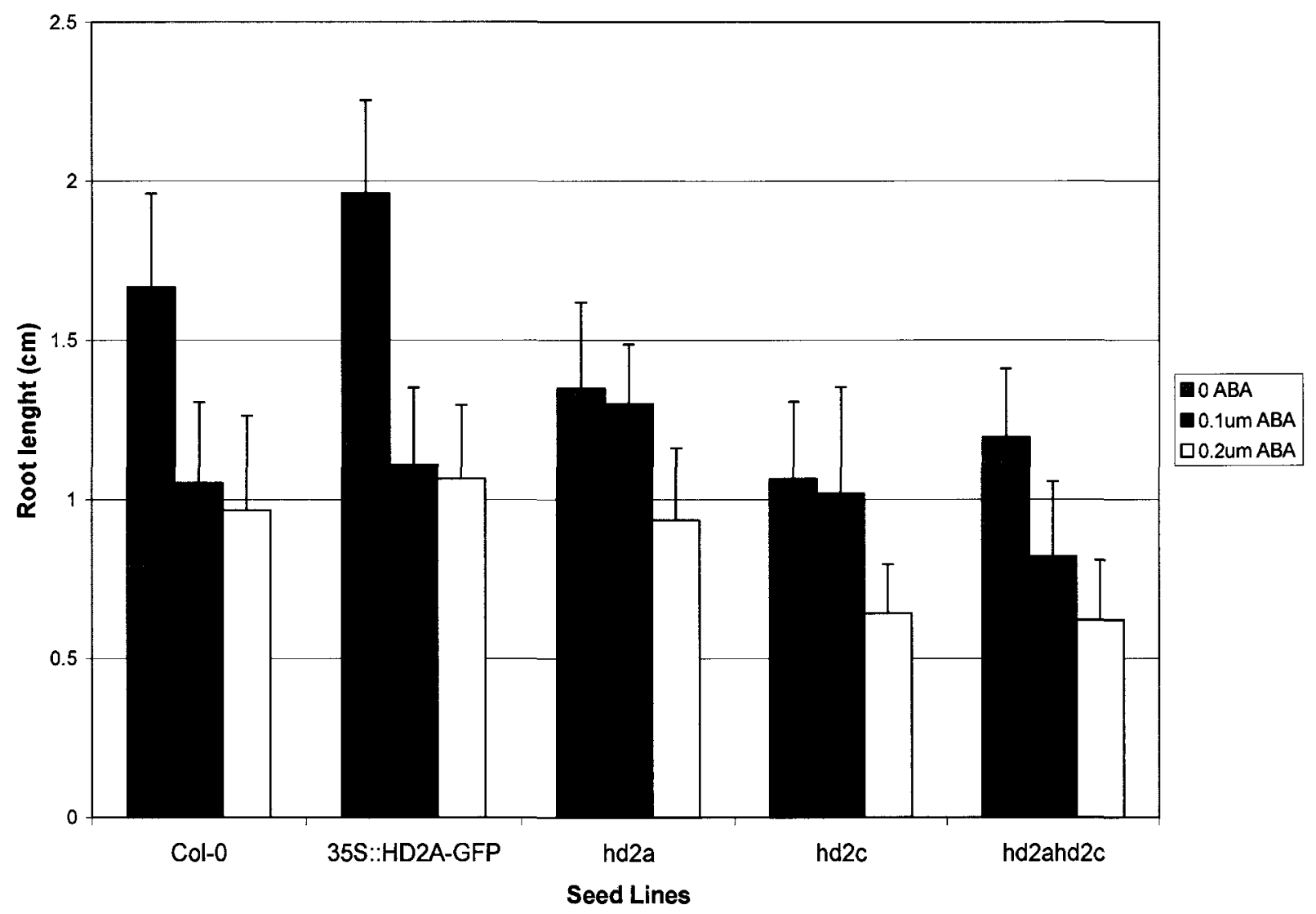


3.6.3 Relationship of the glucose/HD2 Pathway Relative to Abiotic Stress Pathways

Since there is evidence of cross-talk among signaling pathways regulating response to $A B A$ and various stresses (drought, salinity, and cold) (Finkelstein et al.,2002; Xiong et al., 2002) and since It has been found that the ABA-deficient (aba) and ABA-insensitive (abi) mutants have a tendency to exhibit salt insensitivity during germination (Leon-Kloosterziel et al., 1996), we tested the salt sensitivity of the $35 S:: H D 2 A-G F P, h d 2 a, h d 2 c$, and $h d 2 a h d 2 c$ double mutants plants. Our results show that $100 \mathrm{mM} \mathrm{NaCl}$ reduced the germination rate of wild-type, 35S::HD2A-GFP, hd2a, hd2c, and hd2a hd2c double mutants plants while seedling germination inhibited at $150 \mathrm{mM}$ and $200 \mathrm{mM} \mathrm{NaCl}$ (Figure $18 \mathrm{~A} \& \mathrm{~B}$ ). In addition, $150 \mathrm{mM} \mathrm{NaCl}$ and $250 \mathrm{mM} \mathrm{NaCl}$ inhibited root growth of wild-type, 35S::HD2A-GFP, hd2a, hd2c, and hd2a hd2c double mutants plants (Figure 19). In both cases there was no selective difference among the lines. Differences among the lines were less apparent during post-germinative development then during germination.

Mannitol has been also used to assess the response of plants to osmotic stress since it has been shown to increase when plants are exposed to drought stress (Patonnier et al., 1999). As shown in Figure 20 wild-type seeds exhibited growth arrest and further development inhibition at $300 \mathrm{mM}$ mannitol with a germination rate of $32 \%$ while $35 \mathrm{~S}:: H D 2 A-G F P$ seeds displayed decreased sensitivity to mannitol and were able to germinate ( $81 \%$ 
germination rate) and develop healthy cotyledons and true leaves at $300 \mathrm{mM}$ mannitol. A similar phenotype, but to a lesser extent was also observed by the $h d 2 a$ mutants $(62 \%$ germination rate). The $h d 2 c$ mutant seeds were extremely sensitive to $300 \mathrm{mM}$ mannitol ( $30 \%$ germination rate) and exhibited similar phenotype to that of wild-type. $h d 2 a h d 2 c$ double mutants exhibited a moderate sensitivity to $300 \mathrm{mM}$ mannitol ( $71 \%$ germination rate), less than wild-type and $h d 2 c$ mutants. However, $300 \mathrm{mM}$ mannitol had a similar effect on root growth of wild-type, 35S::HD2A-GFP, hd2a, hd2c, and hd2a hd2c double mutants plants (Figure 21). These results were very similar to the results obtained with salt stress. Under both salt and osmotic stress the differences among the mutant lines were less pronounced during postgerminative development than during germination. Furthermore, the differences in germination among the lines were retained on exposure to increasing levels of abiotic stress. The data suggests that the $H D 2 A$ and HD2C genes play opposing roles in processes that are central to seed germination and upstream of the abiotic stress response pathways. 
Figure 18. Phenotypic analysis of $35 \mathrm{~S}:: H D 2 A-G F P$, hd2a, hd2c, and hd2a hd2c (double mutants (D.M)) under abiotic stress conditions. $100 \mathrm{mM} \mathrm{NaCl}$; $150 \mathrm{mM} \mathrm{NaCl}, 200 \mathrm{mM} \mathrm{NaCl}$ were used as an abiotic stress condition. Col-0 wild-type was used as a control for this study. Seeds were germinated on minimal media supplemented with $100 \mathrm{mM} \mathrm{NaCl} ; 150 \mathrm{mM} \mathrm{NaCl}, 200 \mathrm{mM} \mathrm{NaCl}$ in the growth incubator under continues florescence light (24 hours).

A) Seedlings were observed under the WDS 54 Nikon JAPAN microscope after 4 days of germination. All seeds displayed growth arrest at $150 \mathrm{mM} \mathrm{NaCl}, 200 \mathrm{mM} \mathrm{NaCl}$.

B) Germination rate analysis. Effect of $\mathrm{NaCl}$ on germination rate was scored. $150 \mathrm{mM}$ and $200 \mathrm{mM} \mathrm{NaCl}$ inhibited germination and caused growth arrest of the wild-type, 35S::HD2A-GFP, hd2a, hd2c, and double mutants (D.M) seeds. Values are average of 3 biological replicates $(n=3)$. Error bars represent standard deviation. 
A)

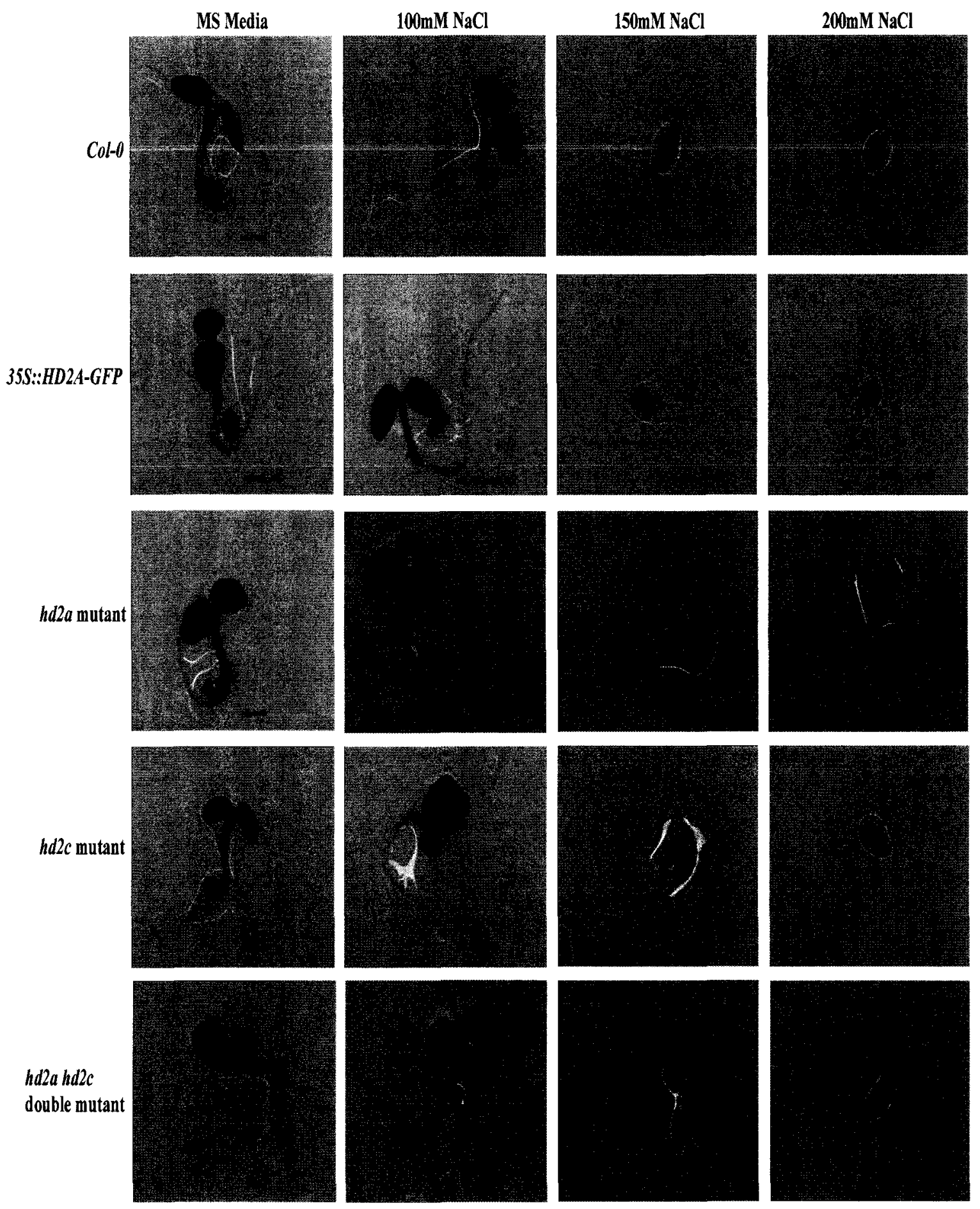


B)

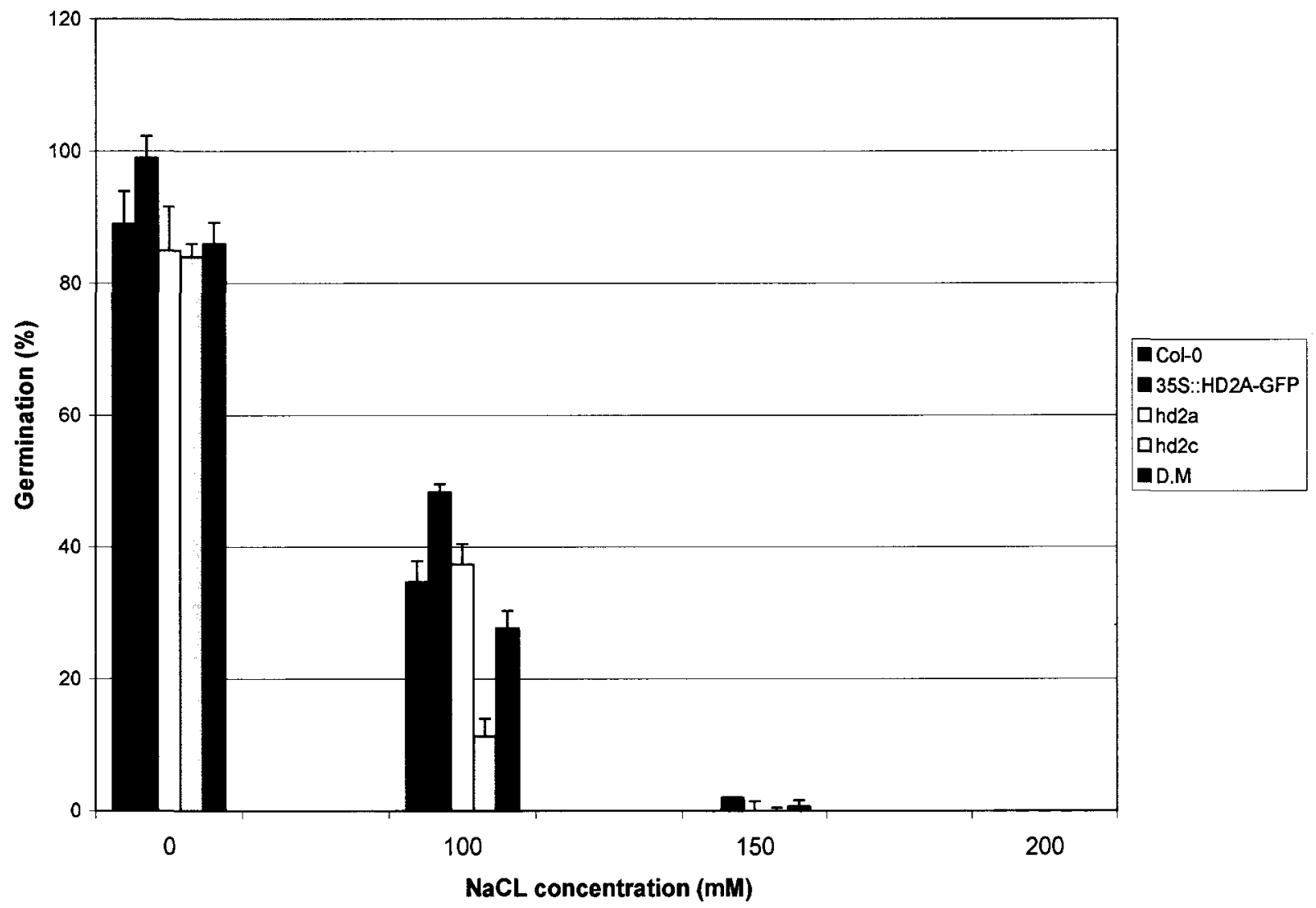


Figure 19. Root phenotypic analysis of $35 \mathrm{~S}:: H D 2 A-G F P, h d 2 a, h d 2 c$, and hd2a hd2c (double mutants (D.M)) under abiotic stress conditions. $100 \mathrm{mM}$, $150 \mathrm{mM}$ and $200 \mathrm{mM} \mathrm{NaCl}$ were used as an abiotic stress condition. Col-0 wild-type was used as a control for this study. Seeds were germinated on minimal media supplemented with 0 (MS media), $100 \mathrm{mM}, 150 \mathrm{mM}$ and $200 \mathrm{mM} \mathrm{NaCl}$. All seeds showed a reduced root length under $150 \mathrm{mM}$ and $200 \mathrm{mM} \mathrm{NaCl}$ in the growth incubator under continues florescence light (24 hours). Root length was then observed after 5 days of germination.

A) All seeds showed a reduction in root length under $150 \mathrm{mM}$ and $200 \mathrm{mM} \mathrm{NaCl}$.

B) Root length measurements analysis. Values are average of 3 biological replicates $(n=3)$ and error bars represent standard deviation. 
A)

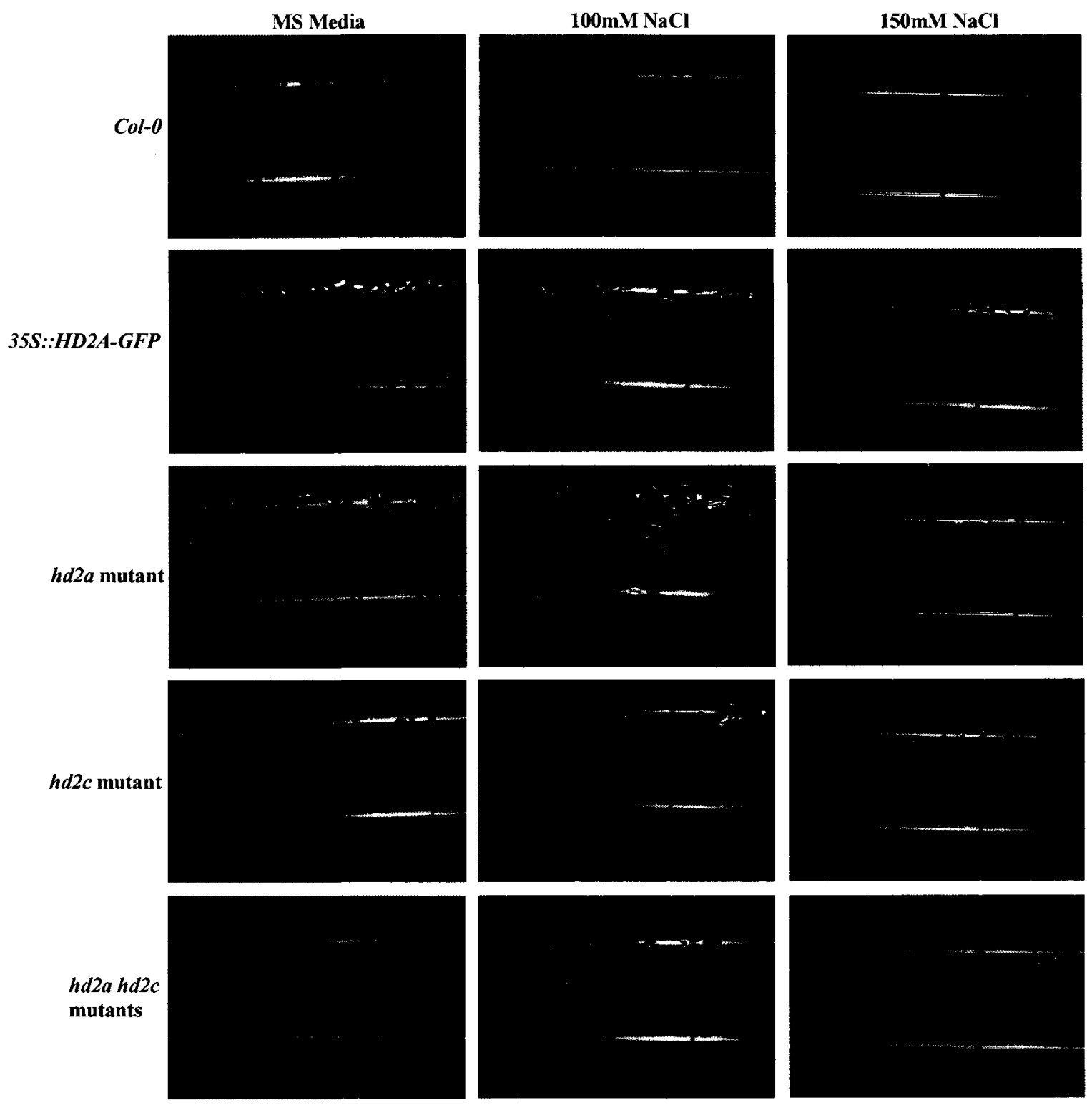


B)

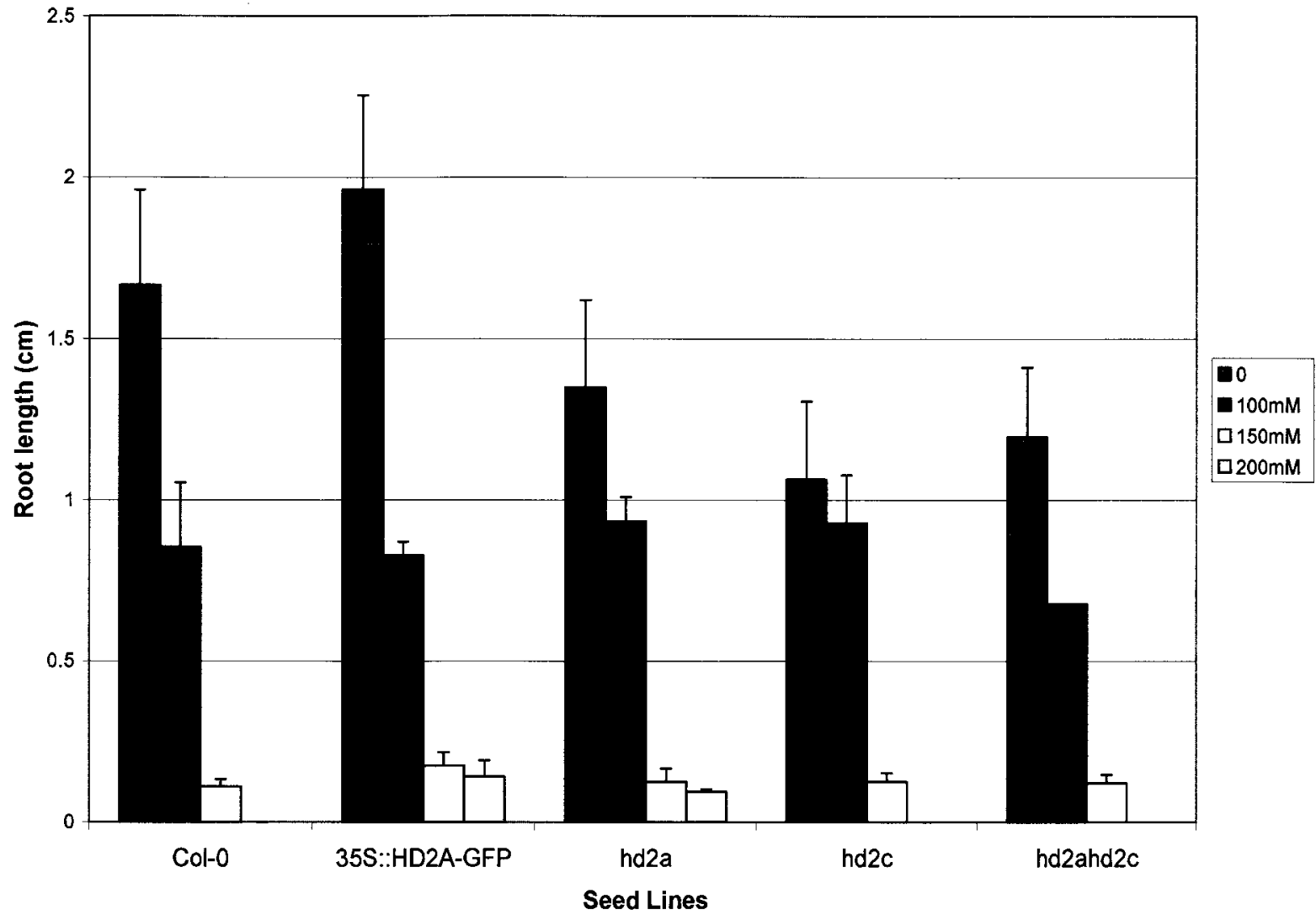


Figure 20. Phenotypic analysis of $35 \mathrm{~S}:: H D 2 A-G F P, h d 2 a, h d 2 c$, and $h d 2 a$ hd2c (double mutants (D.M)) under abiotic stress conditions. $250 \mathrm{mM}$ and $300 \mathrm{mM}$ mannitol were used as an abiotic stress condition. Col-0 wild-type was used as a control for this study. Seeds were germinated on minimal media supplemented with 0 (MS media), $250 \mathrm{mM}$ and $300 \mathrm{mM}$ mannitol in the growth incubator under continues florescence light (24 hours).

A) Seedlings were observed under the WDS 54 Nikon JAPAN microscope after 4 days of germination. Wild-type seeds exhibited growth arrest and further development inhibition at $300 \mathrm{mM}$ mannitol while 35S::HD2A-GFP seeds displayed decreased sensitivity to mannitol and were able to germinate and develop healthy cotyledons and true leaves at $300 \mathrm{mM}$ mannitol. A similar phenotype, but to a lesser extend was also observed by the hd2a mutants. The hd2c mutant seeds were extremely sensitive to $300 \mathrm{mM}$ mannitol and exhibited similar phenotype to that of wild-type. $h d 2 a h d 2 c$ double mutants exhibited a moderate sensitivity to $300 \mathrm{mM}$ mannitol, less than wild-type and $h d 2 c$ mutants

B) Germination rate analysis. Effect of Mannitol on germination rate was scored. Wild-type seeds exhibited germination rate of $9 \%$ at $300 \mathrm{mM}$ mannitol, while 35S::HD2A-GFP seeds displayed decreased sensitivity to mannitol and were able to exhibit a germination rate of $40 \%$ at 300 $\mathrm{mM}$ mannitol. A similar phenotype, but to a lesser extend was also observed by the hd2a mutants which exhibited a germination rate of $27 \%$. The hd2c mutant seeds were extremely sensitive to $300 \mathrm{mM}$ mannitol and had a very low germination rate of $6 \%$ at $300 \mathrm{mM}$ mannitol. $h d 2 a$ hd2c double mutants exhibited a moderate sensitivity to $300 \mathrm{mM}$ mannitol and had a germination rate of $31 \%$. Values are the average of 3 biological replicates $(n=3)$. Error bars represent standard deviation. 
A)

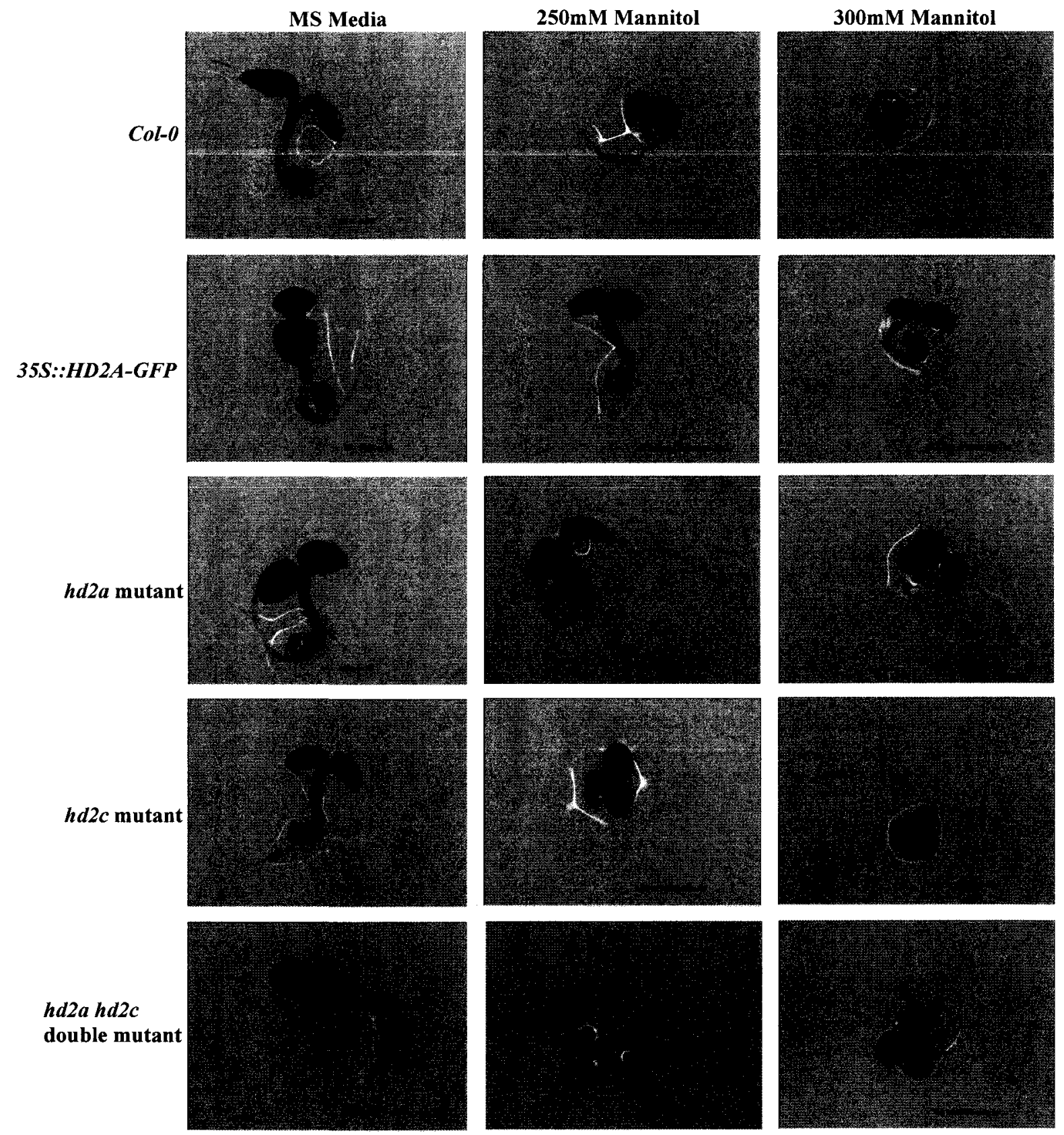


B)

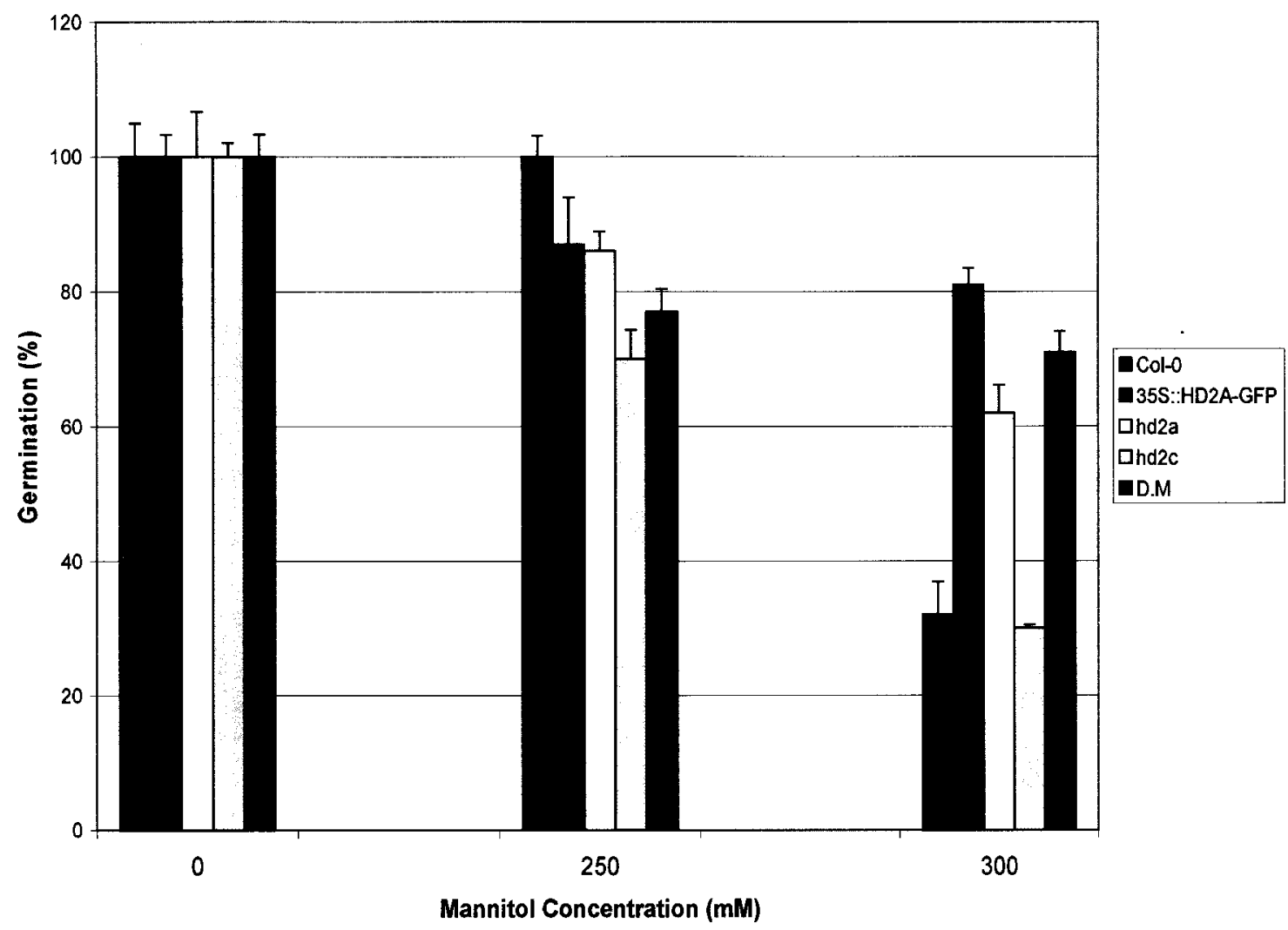


Figure 21. Root phenotypic analysis of 35S::HD2A-GFP, $h d 2 a, h d 2 c$, and hd2a hd2c (double mutants (D.M)) under osmotic stress conditions. $250 \mathrm{mM}$ and $300 \mathrm{mM}$ mannitol were used as an osmotic stress condition. Col-0 wildtype was used as a control for this study. Seeds were germinated on minimal media supplemented with 0 (MS media), $250 \mathrm{mM}$ and $300 \mathrm{mM}$ mannitol $\mathrm{NaCl}$ in the growth incubator under continues florescence light (24 hours). Root length was then observed after 5 days of germination

A) All seeds showed a reduction in root length under $250 \mathrm{mM}$ and $300 \mathrm{mM}$ mannitol.

B) Root length measurements analysis. Values are average of 3 biological replicates $(n=3)$ and error bars represent standard deviation. 
A)

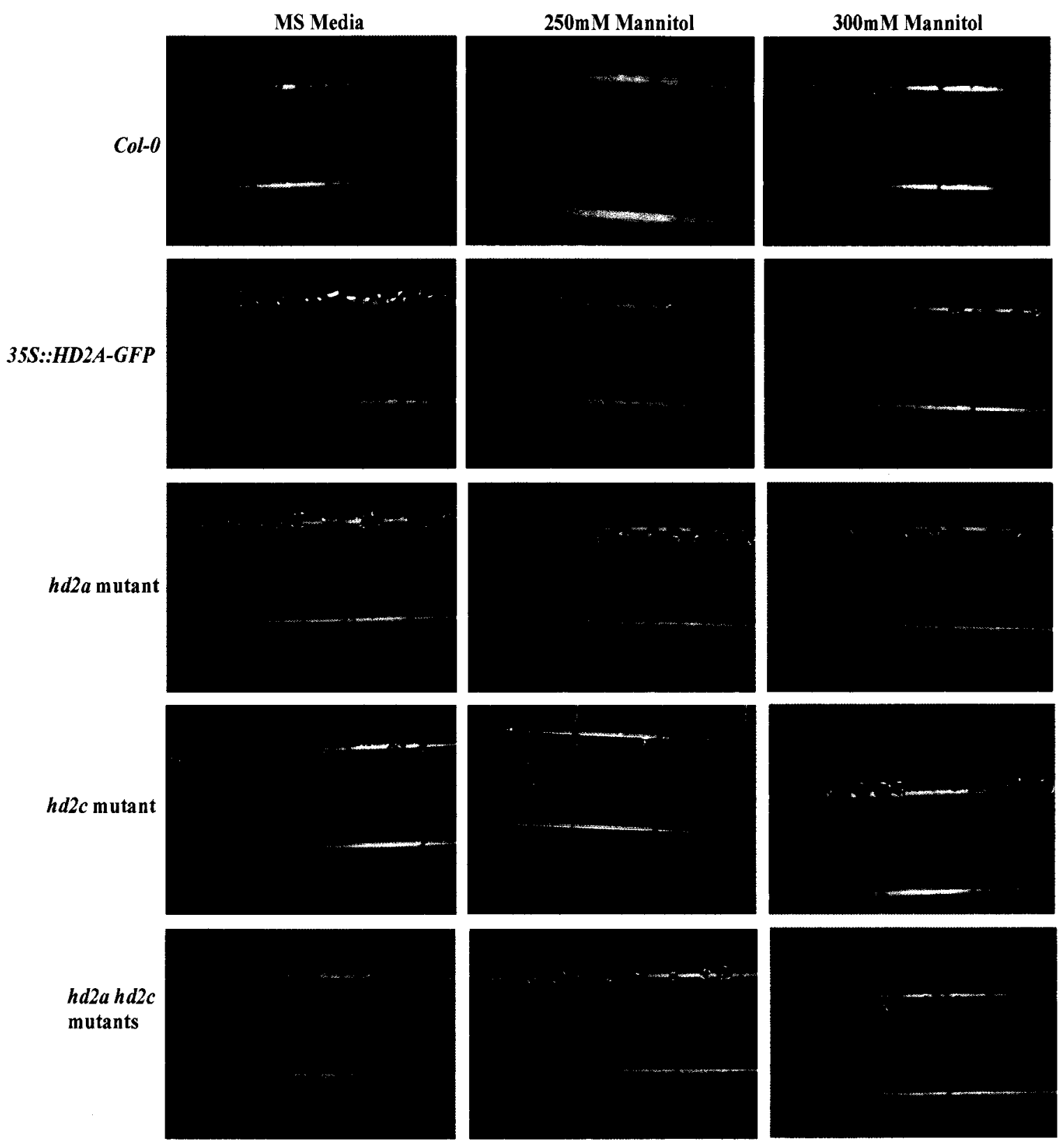


B)

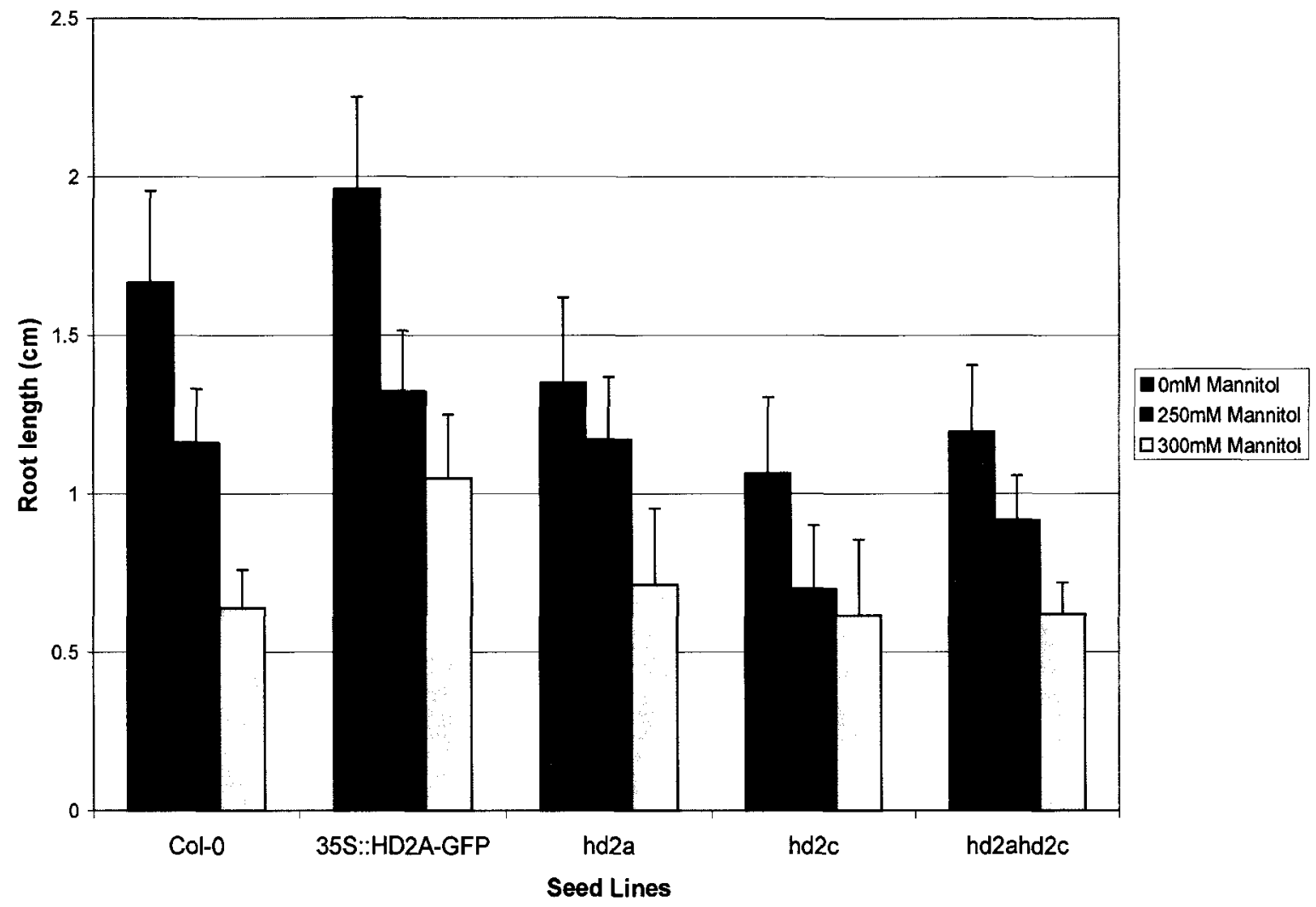


3.7 The HD2C Promoter Is Not Responsive to Glucose In Wild-type Background

Our finding that $H D 2$ induction by glucose is mediated through an AtHXK1-independent pathway rather than AtHXK1-dependent pathway led to the hypothesis of a direct glucose induction through $H D 2$ promoter motifs that have been identified previously in the promoters of glucose responsive genes. Thus, we have investigated the induction of HD2 promoter activity upon soluble carbohydrates treatment with transgenic seedlings expressing AtHD2C::GUS since AtHD2C::GUS seeds were available to perform this analysis from Keqiang Wu (West Virginia University). AtHD2A::GUS lines are also under construction but were not available at the time of this thesis.

AtHD2C::GUS expression patterns upon soluble carbohydrate treatment were analyzed. Our data indicated that AtHD2C::GUS expression was high in all tissues under all treatments as shown in Figure 22 and Figure 23. Thus, we performed T-test statistical analysis to identify whether the difference between the control and experiments is significant. The T-test analysis $(\mathrm{P}<0.14$ (glucose), $\mathrm{P}<0.24$ (fructose), $\mathrm{P}<0.17$ (sucrose)) showed that there is no significant difference between the control and soluble carbohydrate treatment as shown in Figure 23.

These results were expected as they are also consistent with our semiquantitative RT-PCR and $\mathrm{PPCR}$ in which $H D 2 C$ induction upon soluble carbohydrate treatment is not as significant as $H D 2 A$ and $H D 2 D$ induction, however, since AtHD2C::GUS seeds were available we put them to the test. The important study would be to investigate the induction of HD2 promoter 
activity upon soluble carbohydrates treatment in AtHD2A::GUS and AtHD2D::GUS promoters since they were shown to be specific to the glucose response in wild-type background.

This study is not precise and might include some artifacts since the HD2C promoter was cloned into the pCAMBIA 1302 vector which has a $35 \mathrm{~S}$ constitutive promoter in the opposite direction of the cloning sites which might have contributed to the constitutive and high expression of $H D 2 C$. Thus, this experiment needs to be repeated with promoter cloned in a different vector that does not include a $35 \mathrm{~S}$ promoter site to obtain more precise results. 


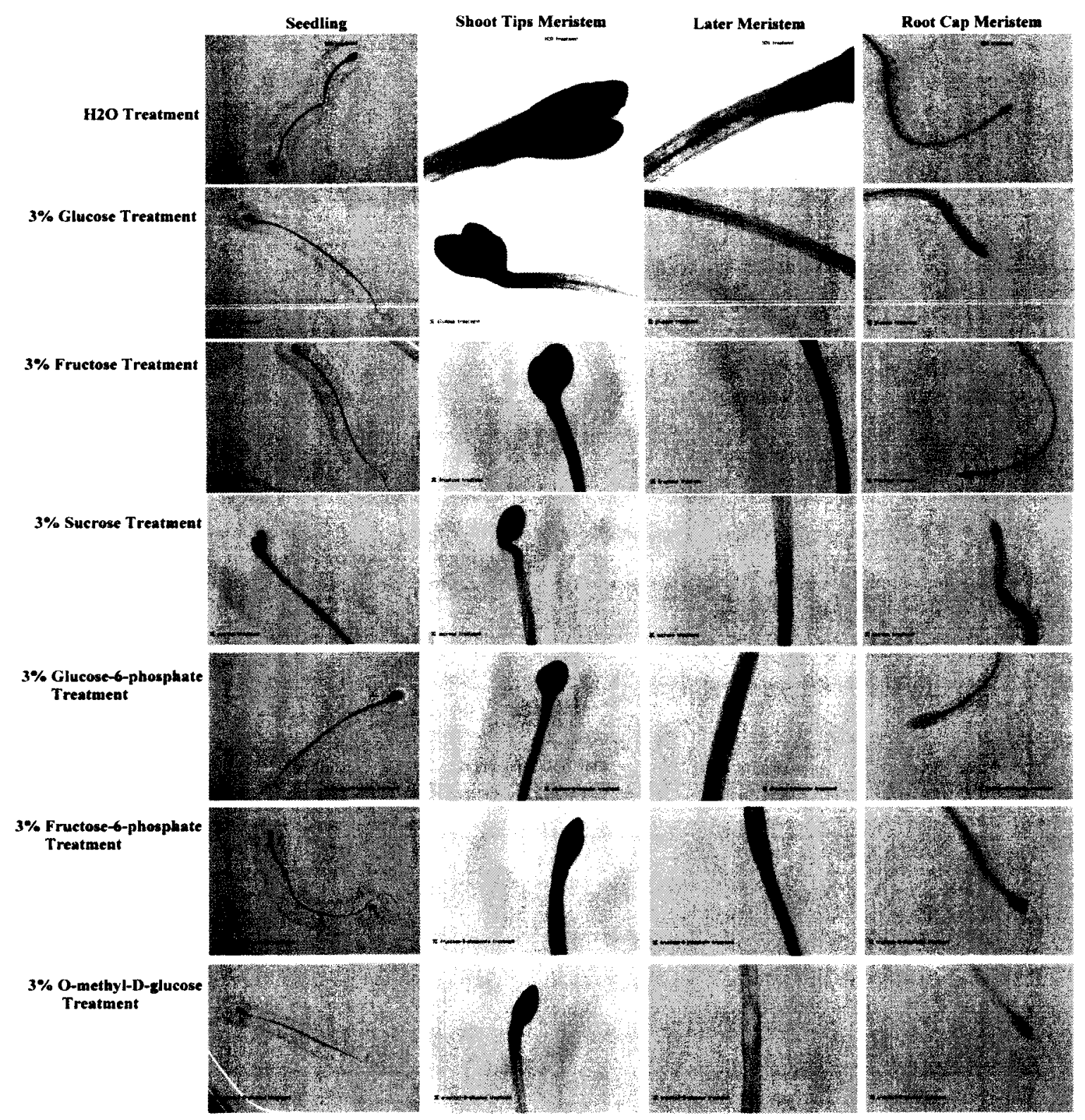

Figure 22. Histochemical GUS staining of AtHD2C::GUS transgenic plants. Seedlings were treated with $3 \%(\mathrm{WN})(166 \mathrm{mM})$ soluble carbohydrate solutions and stained with $1 \mathrm{mM}$ X-Glc. 


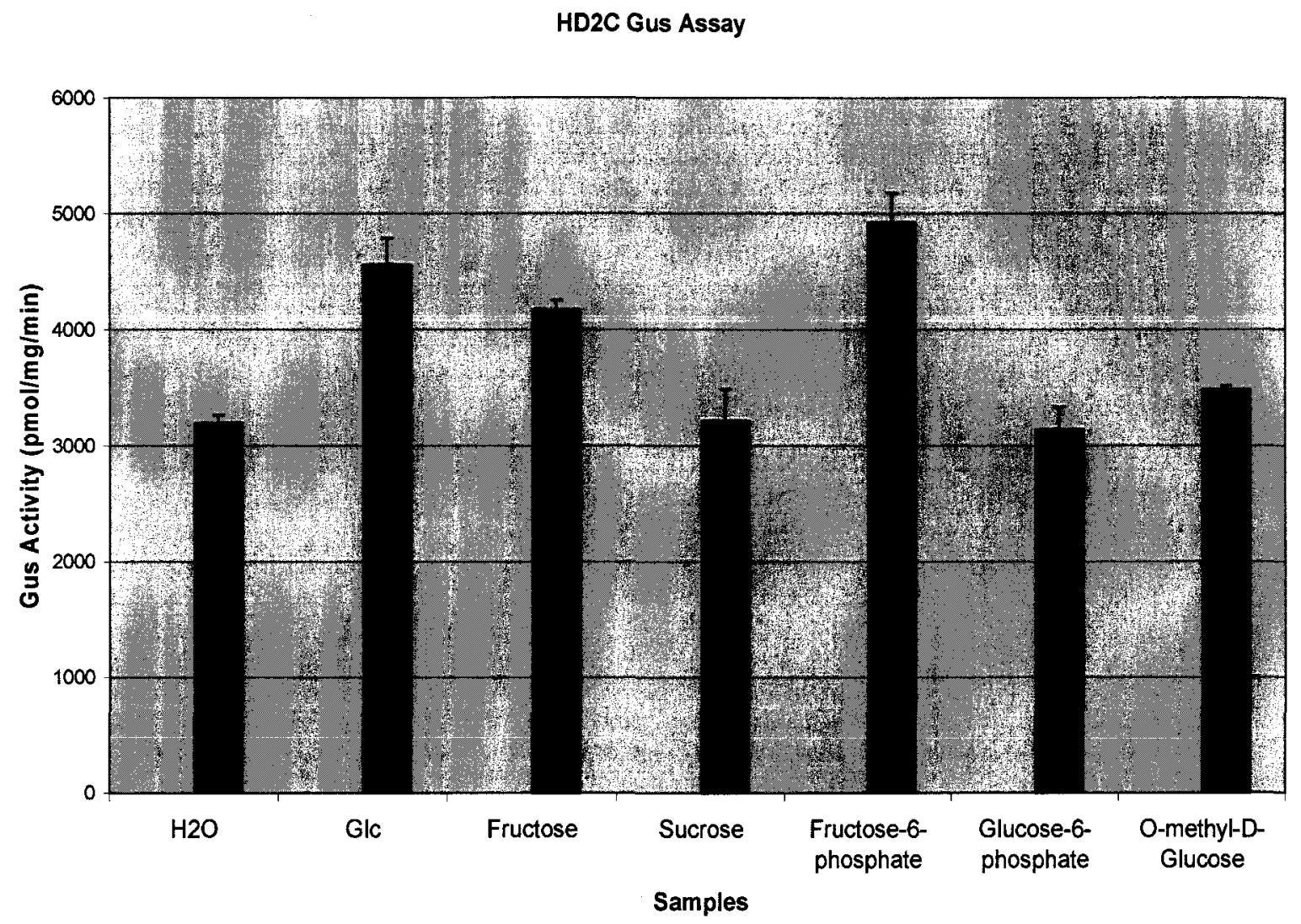

Figure 23. Quantitative analysis of GUS specific activity in AtHD2C::GUS transgenic plants. Plants were germinated in the light on $1 / 2 \mathrm{MS}, 0.8 \%$ phytoagar for 24 hours after 48 hours of stratification at $4^{\circ}$ Celsius. Plants were then incubated in the dark for four days. After four days, plates were sprayed with solutions of $\mathrm{H}_{2} \mathrm{O}$ and $3 \%(\mathrm{w} / \mathrm{v})$ sugar solutions. GUS activity was reported as pmol 4-methylumbelliferone/mg protein/min. Values are the mean of three replicates. Error bars represents the standard deviation of three replicates. 


\section{CHAPTER 4}

\section{DISCUSSION}

Histone deacetylases are an important class of enzymes that are involved in chromatin remodeling and the regulation of gene expression by controlling the level of acetylation of the amino-terminal domains of histones (Aalfs and Kingston, 2000). Many internal and external factors are involved in the regulation of chromatin remodeling. The ability to detect physiological stimuli and transfer the proper response to chromatin is critical to all cellular functions. Distinct signal transduction pathways are activated upon receiving external signals which results in the induction or repression of a specific set of genes. Sugars are known to act as signal molecules in plants resulting in the regulation of certain gene expression (Farrar, 1991; Sheen, 1994; Koch, 1996; Jang and Sheen, 1997; Smeekens and Rook, 1997). Sugars play a vital role in the control of many aspects of the plant life cycle such as germination and seedling development (Moore et al., 2003)

The goal of this study was to understand in greater detail the functions of histone deacetylase 2 (HD2) during plant development in relation to chromatin remodeling under modulation of the external sugar environment. This study shows that among the four members of the $H D 2$ family, $H D 2 A$ and $H D 2 D$ are the most responsive to glucose, fructose and sucrose, whereas the response of $H D 2 B$ and $H D 2 C$ is variable. Further examination of the $H D 2$ promoter elements revealed the presence of telo-box motifs within the promoters of $H D 2 A$ and $H D 2 D$ but not $H D 2 B$ or $H D 2 C$ which may be responsible for the glucose selective 
inducibility (Appendix II). Examination of the glucose inducibility of the HD2 genes in the gin2-1 mutant revealed that the glucose inducibility of the HD2 family occur via a HXK1-independent signal transduction pathway. Furthermore, examination of $h d 2 a, h d 2 c$ and $h d 2 a / h d 2 c$ null mutants revealed distinct functional roles for $H D 2 A$ and $H D 2 C$ during seed germination. The implications of these findings are discussed in relation to sugar signal transduction and embryo germination.

\subsection{Sugars and ABA Signal Transduction Pathways in Embryo Development}

Fine control of developmental timing and spatial patterning is vital for development in organisms. Developmental phases are characterized by phasespecific genes. Transition between phases requires the down-regulation of the previous phase-specific genes and the up-regulation for the subsequent phasespecific genes (Kermode, 1990).

Studies have demonstrated the role of sugars and $A B A$ signal transduction pathways in embryo development and seed maturation (reviewed in Finkelstein and Gibson, 2002). Both sugars and ABA have also been implicated in embryo transition to seed maturation through cell division, and cell enlargement (Wobus and Weber, 1999A;1999B) In higher plants, embryo maturation continues after the completion of pattern formation by accumulation of seed reserves and acquisition of desiccation tolerance (Goldberg et al., 1994; McCarty et al., 1995). The accumulation of seed reserves and storage products are mediated by the sugar ratio (hexoses to sucrose) (Wobus and Weber, 1999A; 1999B) as well as 
ABA level (Finkelstein et al., 2002; Wobus and Weber, 1999B; Rock and Quatrano, 2001). The expression and biosynthesis seed storage protein (SSP) and late embryogenesis abundant (LEA) genes which may be involved in acquisition of desiccation tolerance are induced by ABA (Dure, 1993).

Seeds have mechanisms that inhibit germination under conditions of biotic stress (Carles et al., 2003) to ensure seedling development. Studies have identified genes that are implicated in this response such as $A B \mid 4, A B I 5$, and ABA2. Mutants of those genes are able to germinate at inhibitory osmotic and salt concentrations (Quesada et al., 2000; Carles et al., 2003). However, Carles et al., (2003) has shown that such mutants die within two weeks after germination, demonstrating that proper response to potential environmental stresses is essential for seedling survival and that $A B \mid 4$ and $A B \mid 5$ genes play an important role in this process. Furthermore, germination is known to be regulated by both the growth potential of the embryo in the seed and restrictive properties of the seed coat (Bentsink and Koornneef, 2002). Dekkers et al., (2004) demonstrated that glucose acts directly on the embryo by showing that the removal of the seed coat leaves the embryo sensitive to inhibition by glucose. In addition, ABA has been shown to be involved in the regulation of glucose inhibition of germination. ABA level within the seed seems to determine the extent of glucose response. For example, ABA biosynthesis mutant aba2-1 displays decrease sensitivity to glucose germination inhibition (Dekkers et al., 2004). The glucose inhibition of germination seems to be affected by the ABA levels present in the seed that might affect the sensitivity to the glucose inhibitory 
pathway. This is proved by the fact that low ABA concentration in combination with glucose increases the glucose response (Dekkers et al., 2004) while decreased ABA levels lead to glucose insensitivity (Ullah et al., 2002; Price et al., 2003). Thus, $A B A$ and glucose signal transduction play critical roles in embryo maturation and seed germination.

Chromatin remodeling has also been shown to be involved in the repression of embryo transition during germination. The $P K L$ gene which codes for a CHD3-chromatin remodeling factor has been found to keep the embryo program in its repressed state during germination (Ogas et al., 1997; Ogas et al., 1999). PKL repression of embryonic traits regulators LEAFY COTYLEDON1 (LEC1), LEAFY COTYLEDON2 (LEC2) and FUSCA3 (FUS3) is mediated through transcriptional regulation (Ogas et al., 1999; Rider et al., 2003). Null mutations in the LEC genes result in cotyledons that displays leaf-like characteristics and various defects in seed development (Meinke, 1992; Keith et al., 1994; Meinke et al., 1994; West et al., 1994; Parcy et al., 1997), while overexpression of LEC1 or LEC2 results in miss- expression of embryonic characteristics in vegetative tissues (Lotan et al., 1998; Stone et al., 2001). The transcript level of $L E C 1$ and $L E C 2$ in germinating $p k l$ seedling was shown to be increased significantly over that found in wild-type seedlings (Ogas et al., 1999; Rider et al., 2003) indicating that the PKL gene is necessary to maintain the embryo traits in its repressed state.

In addition, previous studies have shown that the histone deacetylase $H D 2 A$ gene is also essential for proper embryo development. Studies on the 
spatial expression patterns of $H D 2 A$ in Arabidopsis thaliana indicated selective expression in meristematic and embryonic cells (Zhou et al., 2004). Moreover, over-expression of $H D 2 A$ resulted in developmental abnormalities such as abnormal leaves, delayed flowering, and aborted seed development indicating that proper expression of $H D 2 A$ is critical for embryo developmental processes and that $H D 2 A$ may play a role in the regulation of gene expression essential for embryogenesis and embryo development (Zhou et al., 2004). Another histone deacetylase that is selectively induced in germinating seedlings is the Rpd3/HDA1 class I gene, HDA19 (Alinsug et al., 2009). Moreover, Tian et al., (2005) indicated that HDA19 acts as a global repressor of gene expression to allow proper plant development and stress responses (Tian and Chen, 2001). HDA19 proteins are nuclear and may be associated with condensing chromatin during mitosis of dividing cells (Fong et al. 2006) as well as repression of the embryonic traits mediated by the ABA pathway during germination (reviewed by Chinnusamy et al., 2008). Repression of both HDA6/HDA19 the rpd3 type histone deacetylases together resulted in arrested growth after germination and expression of somatic embryos and expression of embryo marker genes (Tanaka et al., 2008). These findings illustrate that chromatin remodeling involves histone deacetylation to repress the embryonic pathways during germination as well as prevent germination during periods of stress through the ABA signaling pathway (Chinnusamy et al., 2008). However, the relationship between sugar sensing, signal transduction and histone deacetylases was not linked or investigated. 
Germination is a highly regulated process that is influenced by many factors including interactions between plant hormones such as ABA, glucose, GA, and ethylene. Glucose and ABA have been shown to prevent seed germination and $A B A$ deficient/glucose insensitive and $A B A$ insensitive mutants show reduced seed dormancy (Koornneef et al., 1982, 1984). On the other hand, GA has been shown to stimulate seed germination in which GA-deficient mutants (ga1, ga2, and ga3) are incapable of germination (Debeaujon and Koornneef, 2000; Bentsink and Koornneef, 2002). Germination assay studies of several sugar effects on seed germination inhibition revealed that glucose, sucrose, and 3-OMG had pronounced effect on seed germination. The inhibitory effect of 3OMG which is taken up by plant cells but not further phosphorylated by HXK (Cortes et al., 2003) suggests that HXK activity or further glucose metabolism is not required to elicit the germination response (Price et al., 2003; Dekkers et al., 2004) and that sugar effects on the germination process is mediated through a HXK1-independent signaling pathway. In addition, sugar-insensitive mutants are resistance to elevated sugar levels with respect to early seedling growth. This developmental block depends on HXK activity, $\mathrm{ABA}$ biosynthesis and $A B / 4$ signaling (Rolland et al., 2002). However, Price et al., 2004 show that HXK and $A B / 4$ are not involved in glucose inhibition of germination, indicating that a different glucose signaling pathway precedes the HXK1/ABA/ABI4 signaling cascade during germination. How glucose represses seed germination is unknown, however, it has been speculated that a similar mechanism to that of yeast in which sugar sensing is mediated by cell membrane such as SNF3 and 
RGT2 (O"zcan et al., 1996) might exist in plants (Lalonde et al., 1999; Roitsch, 1999; Sheen et al., 1999).

HXK1-independent glucose signaling seem to be upstream of hormone signaling. Dekker et al., 2004 demonstrated that glucose acts on the embryo, while GA acts on the seed coat. In addition, Dekker et al., 2004 also showed that addition of GA, ACC or BR does not relieve glucose inhibition of seed germination. Furthermore, abi4 and WT display similar sensitivity to sugar (Dekker et al., 2004) indicating that glucose inhibition of germination via HXK1independent glucose signaling is upstream of the hormone signaling pathway.

Sugars have been shown to be important in controlling the initiation and termination of seed maturation programs and transition from embryo maturation to active vegetative growth during seed germination (Tsukagoshi et al., 2007). Sugars play various functions in seed germination; for example, sugar repress the embryo maturation genes via the transcriptional repressor, HIGH LEVEL EXPRESSION OF SUGAR-INDUCIBLE GENE 2 (HSI2), to prevent expression of the seed maturation programs during seed germination (Tsukagoshi et al., 2007) probably through chromatin remodeling involving histone deacetylases (Tanaka et al., 2008). Suppression of embryogenesis-related genes during germination via HDAC has been reported (Tanaka et al., 2008).

Moreover, sugar has been shown to be involved in cell cycle progression. The expression of cyclin proteins correlate with the level of endogenous sugars and the length of $\mathrm{G} 1$ and $\mathrm{G} 2$ phases could be lengthened or shortened by sugar application or removal (Hartig and Beck, 2005). This suggests that sugars plays 
roles in coordinating the genes involved in cell cycle progression which also have an impact on plant tissue and organ development during germination. Sugars have been shown to play a direct role in the re-entry of inactive cells in G1 arrest into the cell cycle (Riou-Khamlichi et al., 2000; Gutierrez et al., 2002). Cytokinins, auxins, brassonosteroids, gibberrelins and sugars have been shown to regulate the D-type of cyclins (CycD) in the G1-S transition (reviewed by Gutierrez et al., 2002; Inzé and De Veylder 2006). Riou-Khamlichi et al., (2000) showed that glucose or sucrose activate CycD2 through HXK1-dependent signaling and CycD3 through a HXK1-independent pathways in Arabidopsis. Hirano et al., (2008) showed that the formation of complexes between the D-cyclins and the cyclin-dependent kinase (CDK) activity which depend on the availability of sucrose results in the phosphorylation of RETINO-BLASTOMA-RELATED PROTEIN 1 (At RBR1) which results in the de-repression of the S-phase genes and the progression of the $\mathrm{G} 1$ phase to $S$ phase. Furthermore, interactions between retinoblastoma-related proteins (ZmRBR1) and Rpd3-type histone deactylases ( $\mathrm{mmRd} 3 \mathrm{l})$ have been demonstrated in maize indicating the involvement of chromatin remodeling in transcriptional reprogramming (Rossi et al., 2003). These findings suggest the involvement of sugars and chromatin remodeling in the regulation of cell proliferation and differentiation. Thus, glucose signaling is essential for G1-S cell cycle progression and may be an early event in embryo germination; however this connection has not been made yet. 
4.2 Discovery of a New Glucose Signal Transduction Pathway Mediated by HD2 Genes

In this study, we examined the germination of embryo and seedling development to explore the sugar sensing and histone deacetylases relationship using glucose-induced delay in germination as an assay to examine the effect of changes in HD2 family composition. Previous studies have suggested the involvement of $H D 2 A$ in embryo maturation (Zhou et al., 2004) and involvement of $H D 2 C$ in ABA signaling (Sridha and $\mathrm{Wu}, 2006$ ).

Our data revealed that the $H D 2$ family members, $H D 2 A$ and $H D 2 C$ play different and opposing roles during seed germination. The use of the AtHXK1 null mutant, gin2-1, revealed that glucose induction of $H D 2 A$ and $H D 2 D$ genes is mediated through a HXK1-independent signal transduction pathway. These two members are co-ordinately regulated and may function redundantly in the glucose-induced delay in germination. Our germination analysis showed that in hd2a mutants, germination is enhanced relative to wild-type seeds. In addition, over-expression of a HD2A-GFP fusion protein showed similar results to that of hd2a mutants. Thus, HD2A-GFP fusion protein may have acted as a competitive inhibitor to the resident $H D 2 A / H D 2 D$ proteins. On the other hand, our data showed that germination was delayed in the $h d 2 c$ mutant relative to wild-type seeds and that this delay is offset in the $h d 2 a h d 2 c$ double mutants. One explanation for this offset in the delay of germination is that the loss of HD2A and $H D 2 C$ in the double mutants may be partially compensated by the redundant 
partners $H D 2 D$ and $H D 2 B$. It is expected that the ratio of $H D 2 A / H D 2 D$ to $H D 2 C / H D 2 B$ may play an important role in modulating the rate of germination. Hence, our germination data seem to conclude that the glucose/HD2A/HD2D pathway acts as an antagonist to an opposing pathway mediated by $H D 2 C$ and possibly $H D 2 B$ that enhance germination. To confirm our finding, a double mutant of the $H D 2 A / H D 2 D$ and $H D 2 C / H D 2 B$ should be created by obtaining available loss-of-function lines in the $H D 2 D$ and $H D 2 B$ genes from seed collections. However, none were available at the time of this study.

The role of $H D 2 A$ in embryo development elevated the possibility that a similar mechanism for the prevention of germination during unfavorable conditions may be used to retain the embryo maturation program in seeds. In this study, we examined the effect of exogenously applied ABA and abiotic stresses (mannitol and $\mathrm{NaCl}$ ) on germination and root growth as an indicator of postgerminative seedling development. Our data showed that the application of ABA and abiotic stresses resulted in the strong inhibition of seed germination and seedling development in all of the mutants and the HD2A-GFP over-expression line. In addition, the relative extent of inhibition of germination rates among these lines were unaltered indicating that $H D 2$ family regulation of germination was upstream of the ABA pathways. However, at advanced post-germinative stages, exogenous $A B A$ has been shown to repress $H D 2 C$ transcript levels. This may result in the accentuation of the ABA inhibitory effects and the tolerances to abiotic stresses (Sridha and $\mathrm{Wu}, 2006$ ). In support to this conclusion a germination assay of $A B A$ effect on the time of wild-type and mutant initiation of 
germination should be performed as a follow up experiment. Based on our analysis, ABA should accentuate delay in $h d 2 c$.

In addition, we examined the effect of exogenously applied glucose on germination and root growth as well. The effect of glucose on root growth was similar among all lines. However, our germination data showed that $h d 2 c$ null mutant were very sensitive to glucose whereas $h d 2 a$ null mutants and $H D 2 A$ GFP line were hyposensitive to glucose. The glucose-induced delay in germination is increased in $h d 2 c$ null mutant but restrained in $h d 2 a$ null mutants and HD2A-GFP line positioning the glucose/HD2A pathway upstream of the HD2C/ABA pathway. Glucose is known to delay seed germination (Dekkers et al., 2004). However, this phenomenon is offset in the hd2a null mutant suggesting that $H D 2 A$ may play a role in glucose repression of seed germination.

Glucose has also been shown to be involved in cell cycle progression. The expression of cyclin proteins correlate to the level of endogenous sugars as well as the length of each G1 and G2 phases (Hartig and Beck, 2005). This suggests that sugars plays roles in coordinating the genes involved in cell cycle progression. Literature has indicated that one of the early events in germination is re-activation of the cell cycle in embryos by glucose through HXK1-ABI4independent signal transduction pathways (Dekkers et al., 2004). The transition from the quiescent embryo to the germinating embryo occurs at the G1-S phase transition in the cell cycle (Georgieva et al., 1994). The transition is characterized by an initial phase of global gene repression followed by the activation of genes required for translation and chromatin remodeling (Nicolai et al., 2006). S-phase 
genes include ribosomal proteins and proliferating cell nuclear antigens. The promoters of those genes are characterized by the presence of a telo-box motif. The telo-box motifs are found in the promoters of glucose responsive genes (Manevski et al., 2000). Nicolaie et al., (2006) showed that arrest of cell division through restriction of sugars involves unbalanced levels of translational repression of genes involved in cell cycle, protein synthesis and chromatin remodelling. Our promoter examination of the $H D 2$ family indicated the presence of the telo-box motifs (aaaccctaa) in the promoters of the glucose-responsive redundant partners, $H D 2 A$ and $H D 2 D$. One mechanism for the co-ordination of transcriptional programming of glucose-inducible genes could be through the regulation of the promoters with telo-box motifs. In the case of ribosomal proteins and histone deacetylases transcriptional and translational reprogramming may occur simultaneously to prepare for the onset of cell division.

Moreover, cell division must be balanced with organized meristematic activity within the germinating embryo. These developmental programs must be strongly imposed to ensure the development of the seedling. Various transcription factors appear to suppress the embryo program through chromatin remodeling such as PKL (Ogas et al., 1999; Rider et al., 2003 ) and AGL15 (Hill et al., 2008) HDA6, HDA19 (Tanaka et al., 2008). At the present time little is known about the mechanisms of HD2 family members action; however, the selective expression and requirement for $H D 2 A$ during embryogenesis, embryo development (Wu et al., 2000; Zhou et al., 2004 ) and germination indicate that $H D 2 A$ could be important for maintaining the embryo maturation phase 
transcriptional program. During germination this program may remain an essential pathway for delaying the rate of germination during unfavorable environmental conditions. The finding that $H D 2 C$ plays an opposing role is not surprising if the members of the HD2 family act together within a mechanism for fine tuning of the rate of germination possibly by balancing the rate of release from the embryo program with the progression of the germination program.

Our data indicates that $H D 2 A$ and $H D 2 D$ early induction by glucose occurs through a HXK1-independent pathway that could be through the telo-box motifs found in their promoters. $H D 2 C / H D 2 B$ are presumed to act downstream of $H D 2 A / H D 2 D$ since their induction by glucose is not to the same extent as HD2A/HD2D. Sridha and Wu (2006) have shown downstream interactions between the ABA pathway and HD2C. Several ABA pathways for signaling in seed germination and post-germinative development have been demonstrated in the literature (Yamagichi et al., 2009). Thus, HD2C may function in a pathway that enhances germination as well as being responsive to inhibition to downstream ABA pathway. The germination pathway could be under multiple controls, thus, whether different HD2 members function selectively in these pathways is still unknown. However, the data from the double mutant seems to suggest that $H D 2 C$ and $H D 2 B$ could be acting redundantly.

GUS reporter constructs were made with the promoters of the HD2A and $H D 2 C$ genes to determine if the elevation in transcript levels by sugars was associated with the telo-box motif in the promoter (Ming Hu, Unpublished). Furthermore, a deletion of the telo-box motif in the HD2A promoter and insertions 
of telo-box motifs in the HD2C promoter were constructed to assess the role of the telo-box motifs. Transgenic seeds with those constructs were not available at the time of the thesis. However, the responsiveness to sugars and ABA will be tested as a follow up to our finding to determine if $H D 2 A$ and $H D 2 C$ are selectively activated. This will offer greater insight into the signal transduction pathway of the HD2 promoters.

4.3 Model for the Fine Control of Germination by the Glucose/HD2 pathway

Since our data showed that glucose inducibility of HD2 members is mediated through HXK1-independent pathway in seedling, the possibility of a direct glucose- $H D 2$ induction at the promoter level through glucose responsive motifs was raised. Thus, promoter sequences comprising $3000 \mathrm{bp}$ upstream of the predicted ATG initiation codon of HD2 genes predicted in Tair (Arabidopsis.org/tools/bulk/sequences/index.jsp) were assembled. Motif elements in glucose-regulated genes identified by Li et al., (2006) were searched by the DNAsis program in an attempt to identify a DNA binding domain that may regulate expression to sugar (sugar responsive element-SRE) . telo-motifs with a recognition sequence of (aaaccctaa) were found in $H D 2 A$ and $H D 2 D$ promoters but not in $H D 2 C$ or $H D 2 B$. The position of the telo-box motif in $H D 2 D$ promoter was found to be downstream of the TATA box as in a number of proliferating cell nuclear antigen (PCNA) genes from plants (Manevski et al., 2000); whereas, the telo-box in the HD2A promoter was found to be upstream of the TATA box as in the promoter of the Ap40 ribosomal protein (Manevski et al., 2000). Thus, 
those members may be regulated at the level of the promoter through the telobox motif.

In addition, since the $h d 2 a$ null mutant enhanced the rate of germination relative to wild-type controls under normal and stress conditions (high concentration of glucose), our germination data indicated that $H D 2 A$ in wild-type seem to play a role in preventing germination, and maintaining the embryo maturation program (i.e. dormant state). Thus, $H D 2 A$ seem to provide a mechanism for preventing germination under unfavorable conditions. Whereas $H D 2 C$ germination data suggested that $H D 2 C$ in wild-type seem to allow the transition of the embryo from its repressed state to undergo germination. This conclusion is based on the observation that the $h d 2 c$ null mutant displayed a delay in seed germination. Dormancy is known to occur at the final stages of seed development and it is a trait of considerable adaptive significance that prevents seed germination under unfavorable conditions.

Studies have shown that seed dormancy is induced by the plant hormone $A B A$ and also inhibits seed germination. Mutants in ABA biosynthesis (aba) or sensitivity (abi) display reduced dormancy (Koornneef and Karssen, 1994; Bewley, 1997). Thus, the balance between $H D 2 A$ and $H D 2 C$ expression may be required for the control and regulation of seed dormancy and germination and act together with $A B A$ but through distinct pathways.

We have developed a model for $H D 2$ function in chromatin remodeling to effect changes in embryo development and germination. The model addresses the major findings of this thesis. Our model suggests that glucose signaling to 
$H D 2 A / H D 2 D$ perceived by the telo-box motifs simultaneously repress the embryo maturation program and initiates the activation of cell cycle progression in embryos resulting in a switch in transcriptional programs to cell division. This may subsequently activate the $A B A$ signal pathway that might be modulated by $H D 2 C$ as the embryo transitions from the germination phase to the postgerminative developmental stage. The $H D 2 C / H D 2 B$ in the germination pathway may be modulated by feedback control of downstream ABA signal pathways. However, the elements that control $H D 2 C / H D 2 B$ are unknown at this time. 
A)

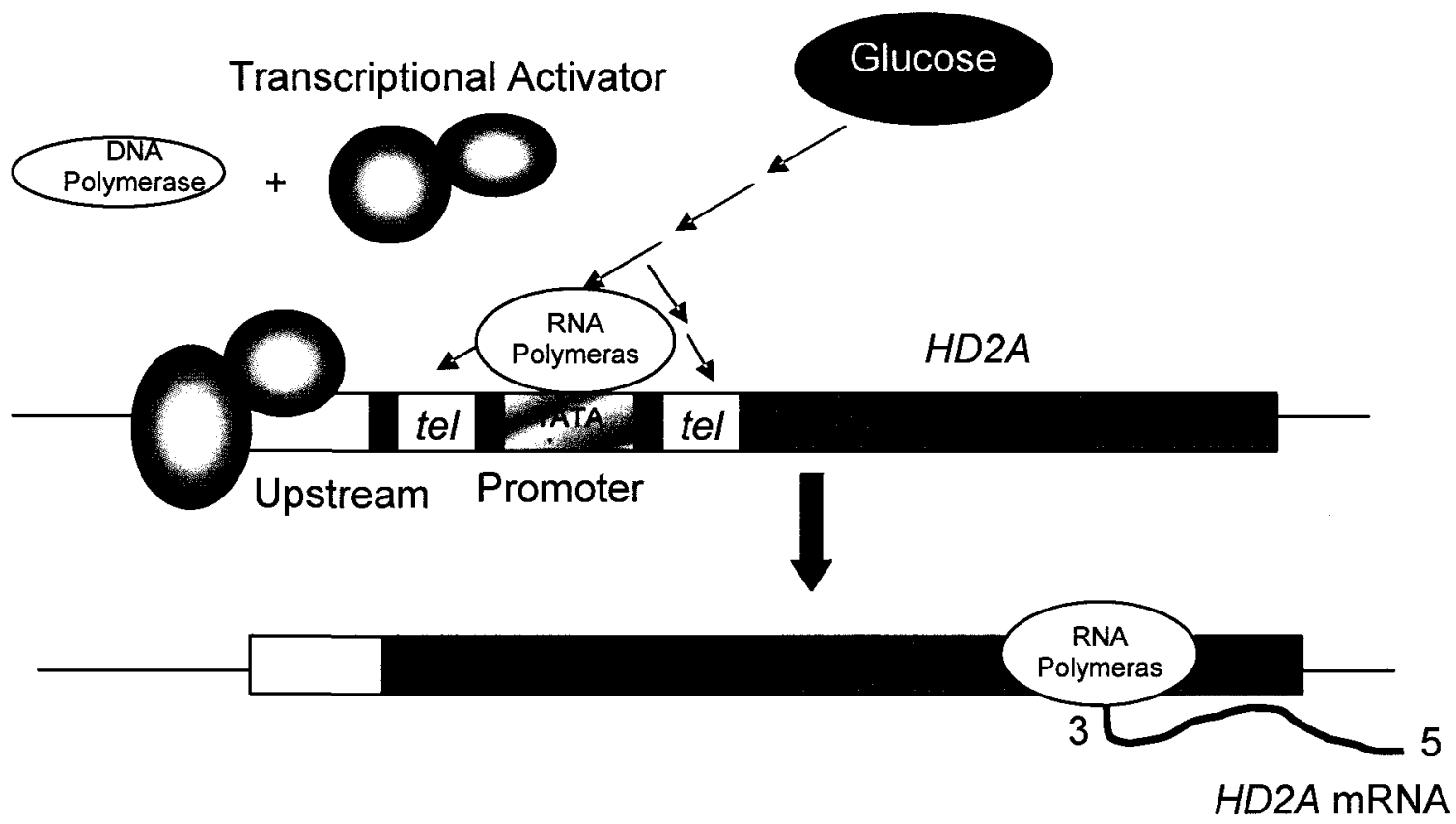

B)

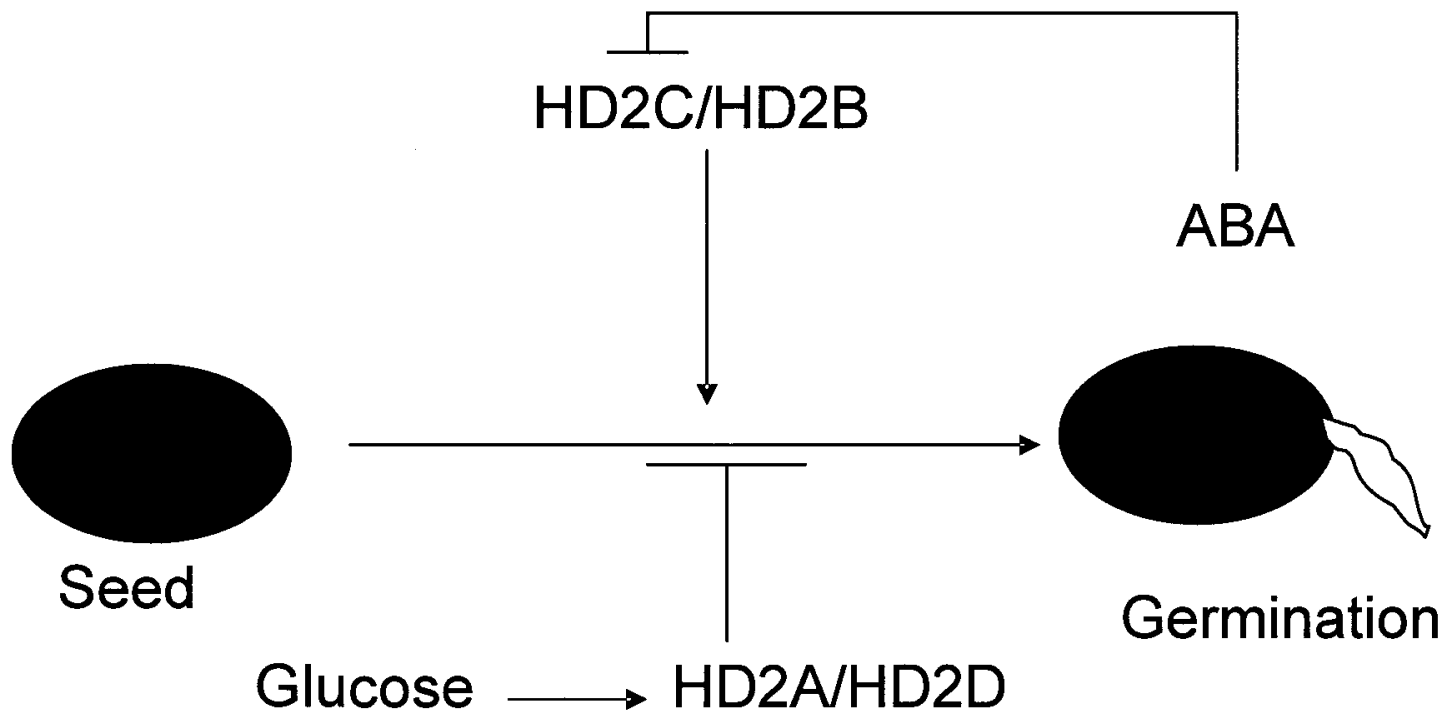

Figure 24: New proposed model of $H D 2$ function. Diagram A propose glucose sensing through the telo-motif in HD2A gene that result in its up-regulation. Diagram $B$ illustrates the role of glucose/HD2A in embryogenesis and $H D 2 C / H D 2 B$ in seed germination and ABA signal pathway. 
Our model does not address the phosphorylation cascade that may be involved in HD2 expression and activation. 12 possible phosphorylation sites were found in $H D 2 A, H D 2 B, H D 2 C$, and $H D 2 D$ of Arabidopsis thaliana (Pandey et al., 2002). Phosphorylation of the maize HD2 complex was shown to be essential for its activity (Lusser et al., 1997). In addition, our model does not address the subcellular localization to the nucleolus of HD2 genes (Lusser et al., 1997; Zhou et al., 2004).

\subsection{Conclusion}

In conclusion, our study suggests a role of $H D 2 A$ in embryogenesis and in glucose - mediating suppression of seed germination. The $H D 2 C$ gene also appears to be involved in the germination pathway and maybe modulated by feedback control of downstream ABA signal pathways. Our data also indicates that the ratio of $H D 2 A / H D 2 D: H D 2 B / H D 2 C$ genes is essential for the fine control of germination rate possibly by balancing the rate of release from the embryo program with the progression of the germination program. Further studies of the telo-motif constructs and possibly null mutants in the $H D 2 D$ and $H D 2 B$ genes will support our conclusion and shed the light into the HD2 role and mechanism in plant development. This model can be applied to other species as similar expression pattern of Arabidopsis HD2 class were found in other species such as Solanum chacoense (Lagacé et al., 2003). 


\section{APPENDIX}

\section{Appendix I}

\section{MUTANT CHARACTERIZATION}

Molecular Characterization of HDTB and HDTD T-DNA Insertion Lines

To identify the possible role of $H D 2 B$ and $H D 2 D$ in addition to $H D 2 A$ and HD2C, on Arabidopsis thaliana development, available collections of Arabidopsis thaliana mutants for $H D 2 B$ (three lines) and $H D 2 D$ (seven lines) were obtained from the Arabidopsis Biological Resource Center (ABRC) and Nottingham Arabidopsis Stock Center (NASC). HD2A and HD2C mutants had previously been isolated by Adam Colville (Colville, 2007). T-DNA insertion lines within the $H D 2 B$ and $H D 2 D$ locus were screened for homozygous plants. Homozygous plants were then screened for the absence of full-length and short-length mRNA transcript. Both short-length and full-length mRNA transcript of all $H D 2 B$ and HD2D T-DNA insertion lines were identified. Thus, no knockout alleles were obtained for $H D 2 B$ or $H D 2 D$ gene. Figure 25 shows an example of one of the $H D 2 D$ T-DNA insertion line in the intron region. Similar results were obtained for the other insertion lines (data not shown).

Introns vary dramatically in size and can be very large. As introns are spliced out of the nascent transcript and are not present in the mature mRNA, a T-DNA in an intron may be spliced out along with the rest of the original intron. It may be inefficient with T-DNA present in an intron, but a proportion of native mature transcript could be produced. This would results in a knock-down, but not a knock out gene. 


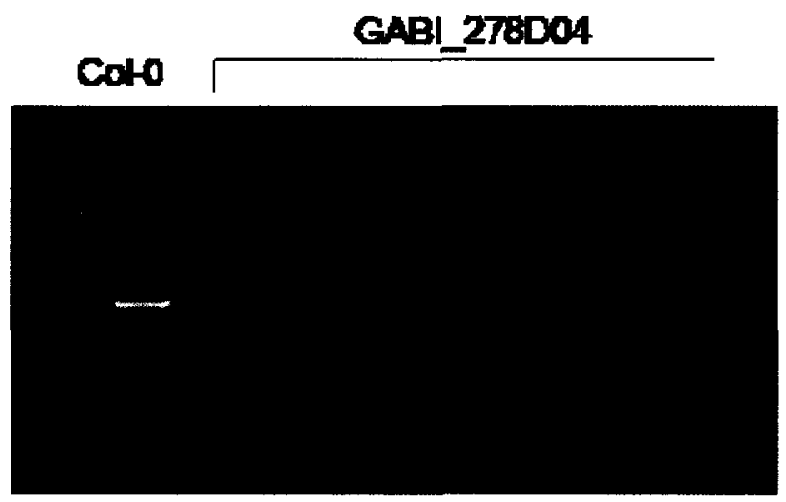

LP+RP pimers

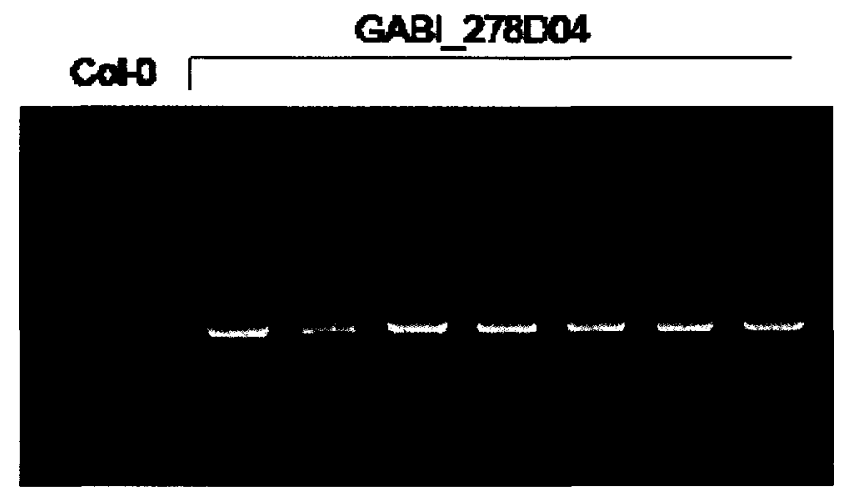

LP+LB primers

B)

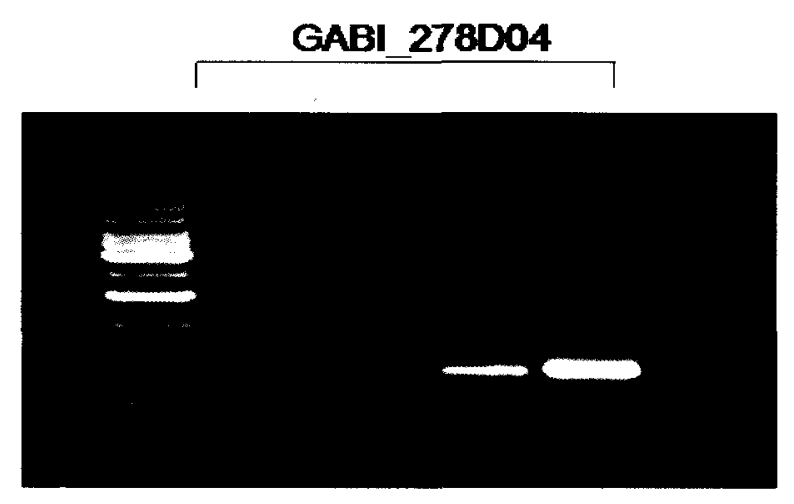

\section{B'.HD2D LP + B'.HD2D RP primers}

Figure 25: A) Genotyping HD2D T-DNA insertion line with LP, RP and LB primers to identify homozygous lines $B$ ) Loss-of-function confirmation detection with $H D 2 D$ full length primers by RT-PCR. A) Lane1 represent DNA extracted from wild-type Col-0, Lane 2-8 represents DNA extracted from GABI_278D04 line. B) Lan1-4 represents one-step RT-PCR of homozygous T-DNA insertion lines of GABI_278D04. 


\section{Appendix II}

\section{BIOINFORMATICS PROMOTER ANALYSIS}

Putative Sugar Motifs Search

DNAlsis program was used to perform a search analysis on the HD2 promoter sequence for putative sugar element motifs. The aim of this search was to find elements known in glucose-regulated genes through which glucoseinduction of $H D 2$ genes could be occurring.

Promoter sequences comprising $3000 \mathrm{bp}$ upstream of the predicted ATG initiation codon of HD2 genes predicted in Tair (Arabidopsis.org/tools/bulk/sequences/index.jsp) were assembled. Motif elements in glucose-regulated genes identified by Li et al., (2006) were searched by the DNAsI program. telo-motifs with a recognition sequence of (aaaccctaa) were found in $H D 2 A$ and $H D 2 D$ promoters. Consensus sequence of (aacccta) was found in the $H D 2 B$ promoter but not HD2C. TEF motifs element with a recognition sequence of CATAAT that are known to interact with the telo-motif, and forms the core of the 16-bp TEF motif, was found in the HD2D promoter sequence. Furthermore, 3 of the 45 mer motifs which comprise 11 of the $16-n t$ TEF motifs were found in the HD2A promoter sequence, two in the HD2B promoter sequence, and two in the $H D 2 C$ promoter sequence.

The telo-motif was initially identified in promoters of genes encoding components of the translational machinery and is classified as the top motif for glucose induced genes (Tremousaygue et al., 1999). The telo-motifs are conserved among different plant species and are found at the 5' region of plant 
genes (Manevski et al., 2000). It is required with other elements such as the TEF, trap40, and Ila/llb elements for high level expression in actively dividing cells in root meristems (Tremousaygue et al., 1999, 2003; Manevski et al., 2000). telomotifs are also known to be enhanced in the promoters of protein and nucleotide synthesis genes (Li et al., 2006). In addition, Li et al., (2006) showed by measuring GUS activity that promoters containing the telo-motif are expressed at highest after 4hours of glucose addition. Quantitative analysis of GUS expression in TEF1TELO3:: GUS transgenic plants expressed a 6.9-fold higher GUS activity in response to glucose treatment compared to mannitol treatment (Li et al., 2006). Moreover, another study by Tatematsu et al., (2005) shows that telo-motif is overrepresented in the promoters of genes up-regulated during axillary bud outgrowth in Arabidopsis, such as ribosomal protein and cell cycle genes. This indicates that the telo-motif play a role in regulating the expression of genes in response to growth stimuli such as glucose and decapitation (Li et al., 2006).

Thus, investigating the role of telo-motifs in HD2 promoters might provide mechanism for the coordinate expression of HD2 genes with other genes involved in plant development. 
Figure 26: Promoter sequence search of telo-box motifs in HD2A (A) and HD2D (B) promoters. Yellow highlight represents TATA box, and underlined sequences represents the telo-box with the sequence of (AAACCCTAA). 


\begin{abstract}
A)
aagcttgcatgcGAAATGAAGAAAATACAAGATAAAAGTAAGTTTCTTACCGAGTGGGCA CGTAAGTCGAATGCAGACCTATCTGCTATACCAACACATTCAATCCACCCATATGAACTC TCAAATTCAGCATCCAAACAATCTGCAGCATAGCGGGCCATTTCATTTGCAAAATGCTGG CGGAAACGCAACTGTTCCTTGTCTGTGCCAAGACGGACAAGGAAAAGATACACTCTCGCA ATGAAGTAGCCTACAGTTTCTTTGTTCACAGTTCCCTATTCAAATACAGTTGCAGGCATC AATAAGCAATTAAGAAACAAAAGGTCTGAGGGAGTGCATACTAGAAGTAAGTAGACGCAG ATGATTACAGATACTGACCTTGGCAACAACTTCGCCAAGGCAAAGTTTTTTCGCAGATTG GCCAGACATTTGTTCCTCTCTTGGAAACATAAGGAtTTCAAAATTTGCTACATCAGAGAA TTTCGGATGTGACTTATTCCCAGGATCAACAAAATGCTCAATTTCTGCCAGCGTGAtTTC ACAAACTCTAAGAAGCCCTTGACGATGAGATATCTGCAAGTAATATGCGTCACTTATTAA AAGCCAGACATAAAAGAAAGAAAAAAGAGTCCCTGATTGAGATAAACACATCCAATCTAT CAAGCAAAGGAATAGAAAATTAAACGATAATCACCTCATTTCTAAAGGTTGACCAATTTG AGCAGCAGCAAAGGGAAATTTCCTCCCATTGTAGTAATACAAGTCCTTCACAAAAATGCC TTGTTAGAGTGTTCTTCGTGTCTGGAGCAATAATCCTTAAGCAAGTGGTCCGTCCGCACT GTAACAAGTTCCAGTTTTCTCATTCTTAACCATTAGATCAGTGAACTGGTCTACATGTCC AGATGCCTTGAGAACAACCTCTGGTGTCACACATGGACAATCCACTTCATACATATTCTC CTTAAGAATGAAATGCTATCTGAGAACAAACCTAAAACAATCTCTACTCTTCTCTATGAA TCATGTCAAATTTGTCTCAATTTCTACTCAAAAGAGAAAGGTGAATGATGTTCCGTACTT ACTTGACGCCAGAAGCTAAGAACATTGGATTTAACAGCACAATCCTTAATCAAATAGTCC GGCGACGTCACGGTTGATTTTGAAAGAAGGGATATAGAACAAACGCCGCTCGAGAGTATT AACCACATTTTGTCGAAATCAGCAGCTCCAaGGCTGCATCAATCGTGAAGCCTTGAGAGC TTGAAGACATTCCCCTGAGCTTCGACGGAGGAAGATTTCGCCTATACTGATTTCCGCCGT TTGAATTTGATCGGAGCATTTTGTTTTTTCACAAACTTTCATATCTTTAACGACGTCGTT AGTACTGAAGAAAAACCCTAAAGAGTAAAGACCCTAACGGCGTCGTATTAAAACCCTAAA ACGGCTTCGTATTAAAACCCTCTGTCTTATAAATAATCTCTTAAAACCCTTTCTTCTTCt cttacattttcagctgctcataaaccctaaaatcctctcttttctcaaccttgattct tagcCGGATCCCGGGCCATGG
\end{abstract}




\section{B)}

TGCAGATAGCCACGTTTCTATTATGTGTATTAACGAAATTTGCTTCTTATTATTATATCAGCCATGAGATT CAATCTTTCTAAATAAAATTGCATTTTCAATCATAACACTTTTTATGGTTATAATATAATTAGGCCACGTG AAGCATCAAAGCGAAGAAGCTCGTATACATACCAGAACAAAAACTAATATTAGGTCCACTCCTATTTTGAT TAGTTTAAAATTAGATATTTCGTTAAACCTCACATAAGTTGGTCATATAATATTTTGCAAAGAGGTTTACT TACGTAATCTTATGCCCAACATAAATAATTGATCTATTTTATGTTATGCTTCGACCCTCGATAAGTCAAGT TTAGCTGCAATAATAGTTCGTGTATTTCTTGATTCTTTGATTTAATACTTATGCAACGTAATTGTCCAAAT GATGTTACTATACTATGCAATTATTGAGGAGACTAATTGCAAAACAAGTTGAAGAGGATGAGATTGGTCAC GTCTCCGGCATCTTTCAGTTATGTCGGCCGTTTGGTCCCATCTTCGCTTTCTGTTCCTGCCATGTGTTTTT TGTATGTTACACCGTGTTTCTATTTGTTTGTAATTAAGAGAGACTTGGCCTTGTACTCTTGTAGGCCTAGC TTTGTTCGTTGAACCTCTTTTTTTTTATTTTTTTGTTCGTCGAACCTTGCAAGAGTCTTATCTCCAGTCT TACCCGGTTTAACTAGCTAGTGCTTAAATAAGTCTTGTTTATAATTTGTAAACTTTCAAAACTAGATATCG TTATATCATCTATCTTATGATAATTAATACAGAAATGAGAAAAGCAACATAAATACATACTACATAATAAA AATACGTAGTTTTCTAACATGCTTTTATTTCACAAAACATAGAAAAAAAACTGTgTgCACTTTGTGAAAAA GATTGTTTTGTTCATGCATTTGTGAATAATACATATCAGATCAATTTTATGTATCGTACTTTGAATTCA TAAAAATTGTCTGATTCATGCATTCAAATCCATTATGAAGTTTTCTGTTACAAATGCATCTTTTAGTAAAA AAGGAAGTATATAATTAAAATTCATTTGAAGTTTTCTGTTACTAATGCATCATGTACATGAATATACTGTA GTAAAATTTCTACTAAAGGGAATGAACTTATTCTCATAATTACTTTGATTTCACAAAGATTACACAAAATC AATTGTGTTGACCAAACTAAAATACTACTACTAAACTTAATTAAAAACAATGATAATCATTGAGTATTTCA AGAGGTGAACACTATATTACAAAGTAACCAATGAAAATAACAAAGAGTCGAGTCCAAACCAAAACCTTAGT CCAAATTGTTTCTACGACCCAGTGGATATAACAATTTCATAAAATTCATAGCCACCGGTTAAACCTGGAC CCAATACCGAACTCACACACATAACTGAAACTCCAGTTCACCTCCTATAGCCGGTTCCCTTCTCTCACACT CTCAGAAATTTCCAAGCAATTCTCACCGTTTCGTTATCTACAAATCCACTATAAATACTTCACTCTTCAG CTTTGTATTATTCTCTTAACATTCCATTACTCTTATCCTTTTACCCTCATCATCCTCCACTATTTACAGTT TTGCCACTCTGACTTTATGCTAGCTTCTTCCGGTGACTGGCGATAACACGAGAGAGCCTTCGCCTTCTCCA AGCTTCATACTCTTCCGACGATAAAGATTAGAAGACATGAAGAACGGTAACCGGAAAACAACGCCGGAAAC AGAGAAGTCTCACCGGAGAAAGTTATCAGAGAAAGCGATGTCGTTTCACGGCAGAGGAACAACGCCGTTAT CAAATCCAGGCGAGCTTCGAAGACCGAAAACGTTACCGGAGTTATTCTCCACCGGTCAAAGCATCACCGTA CCGGAGACGGTTTCACTTCCGCCACGTTTGACGAAACTTTTACTTAACGTGACGGTACAAGGAAGTTTAGG AGCCGTACAAATTATAATCTCGCCGGAATCCACCGTGAGTGATTTAATCGACGCCGCCGTTCGTCAGTACG TTAAAGAAGCTCGCCGGCCATTTTTACCGGAATCTGAACCGTCACGATTTGATCTTCATTACTCGCAGTTT AGTCTTGAGAGTATTGTAAGAGACGAGAAGTTGATATCGCTAGGGTCGAGAAACTTTTTCTTATGTGGTCG GAAAGAGACCGGAGGGTTTATCGGCTGTGGTTTATCGTCGGAATCTTGTTCTAAGGAAGCAGAGAAAGTGG CTAAAACTGGTTTTCATTGGCTCAAATTTATGGGCTTCTAGAAGTTTTTATCATTTTGAATTATTGCTTTT TCAAATTAGTTCCATTCTTTAGAAAAAGgATATAAAAAACATCTGTGTATATTATTTCTATGTAGAAATGA AAATCATTAATTAGTGGTGTATAATGTATTTTAATCAATTATATACCAAGATGATTATATGAAGTTCTAAA GTTTACCAATACTATTTGATGAAATTTTATTTATTATATAGTTGAAAATCTATAAAGCATTATTCGTG GATGAATATAGCCATGAAATGGGTATTATAAACTGAATAGAACATTGTATATTATATATTATCTTTTTATA ATATCTTTTTTGGACCTCTAATAATAGAACTTTTGCATTCTGTTTATGTGATAACGACATTGCCCAACCTT ATCCCTAAAGTCAGACATTTTTGAGTAAACCAATTTGATGCCGTGTTGCTCTTCCTTCTCTTATTTGTTTA ATCAACATTCTTTCTCTTTGATCATTGAAGCAAAGTTAATTACCCAAATCATAGGCAACTCTTATGACATG GCTATAAAATTAAAATATATCTAAAACCCATCAAATAAGGCCGATTCAAAATAAGCCCACTAAAAATAAGG CCCATTAATATACTATTCCAAAAGAAGTAACTAAAAACCCTACCTGTGAAAAGGAAGTTTAAAGGAGTAGT AAGAGTAACCTTATAAATaagccgctccattatttttgtttgtttgaaacaaactctaaaccctaaattc tectctcaagcagccacatct cctcttcactagct 


\section{REFRENCES}

Aalfs, J., and Kingston, R. (2000) What does 'chromatin remodeling' mean? Trends Biochem. Sci., 25:548-555.

Alinsug, M., Yu, C., and Wu, K. (2009) Phylogenetic analysis, subcellular localization, and expression patterns of RPD3/HDA1 family histone deacetylases in plants. BMC. Plant Biol., 9:37.

Aravind, L. (1998) Second Family of Histone Deacetylases. Science, 280:1167a.

Ausio, J., Dong, F., and van Holde, K. (1989) Use of selectively trypsinized nucleosome core particles to analyze the role of the histone "tails" in the stabilization of the nucleosome. J. Mol. Biol., 206:451-463.

Barker, L., KuÈhn, C., Weise, A., Schulz, A., Gebhardt, C., Hirner, B., Hellmann, H., Schulze, W., Ward, J., and Frommer, W. (2000) SUT2, a putative sucrose sensor in sieve elements. Plant Cell, 12:1153-1164.

Bentsink, L., and Koornneef, M. (2002) Seed dormancy and germination. In: Somerville CR, Meyerowitz EM (eds) The Arabidopsis book. American Society of Plant Biologists, Rockville, MD. DOI 10.1199/tab.0050.

Bewley, J. (1997) Seed germination and dormancy. Plant Cell, 9:1055-1066.

Blasing, O., Gibon, Y., Gunther, M., Hohne, M., Morcuende, R., Osuna, D., Thimm, O., Usadel, B., Scheible, W., and Sitt, M. (2006) Sugar and circadian regulation make major contributions to the global regulation of diurnal gene expression in Arabidopsis. Plant Cell, 17:3257-3281.

Boggs, B., Cheung, P., Heard, E., Spector, D., Chinault, A., and Allis, C. (2002) Differentially methylated forms of histone $\mathrm{H} 3$ show unique association patterns with inactive human X chromosomes. Nat. Genet., 30:73-76.

Borisjuk, L., Rolletschek, H., Radchuk, R., Weschke, W., Wobus, U., and Weber, H. (2004) Seed development and differentiation: a role for metabolic regulation. Plant Biol., 6:375-386.

Borisjuk, L., Rolletschek, H., Wobus, U., and Weber, H. (2003) Differentiation of legume cotyledons as related to metabolic gradients and assimilate transport into seeds. J. Exp. Bot., 54:503-512.

Borisjuk, L., Walenta, S., Rolletschek, H.,Mueller-Klieser, W., Wobus, U., and Weber, H. (2002) Spatial analysis of plant metabolism: sucrose imaging within Vicia faba cotyledons reveals specific developmental patterns. Plant J., 29:521-530. 
Buchanan, B., Gruissem, W., and Jones, R. (2000) Biochemistry \& Molecular Biology of Plants, Waldorf MD: American Society of Plant Physiologists.

Cairns, B. (1998) Chromatin remodelling machines: similar motors, ulterior motives. Trends Biochem. Sci., 23:20-5.

Calikowski, T., Meulia, T., and Meier, I. (2003) A proteomic study of the Arabidopsis nuclear matrix. J. Cell Biochem., 90:361-378.

Carles, C., Bies-Etheve, N., Aspart, L., Leon-Kloosterziel, K., Koornneef, M., Echeverria, M., and Delseny, M. (2003) Regulation of Arabidopsis thaliana Em genes: role of ABI5. Plant J., 30:373-383.

Carrozza, M., Utley, R., Workman, J., and Cote, J. (2003) The diverse functions of histone acetyltransferase complexes. Trends Genet., 19:321-329.

Caspar, T., Huber, S., and Somerville, C. (1985) Alterations in Growth, Photosynthesis, and Respiration in a Starchless Mutant of Arabidopsis thaliana (L.) Deficient in Chloroplast Phosphoglucomutase Activity. Plant Physiol., 79:11-17.

Ciereszko, I., Johansson, H., Hurry, V., and Kleczkowski, L.A., (2001) Phosphate status affects the gene expression, protein content and enzymatic activity of UDP-glucose pyrophosphorylase in wild-type pho mutants of Arabidopsis. Planta, 4: 598-605.

Chen, J.G. (2008) Heterotrimeric G-protein signaling in Arabidopsis. Plant Signal. Behav., 3: 1042-1045.

Chen, J.G., and Jones, A.M. (2004) AtRS1 function in Arabidopsis thaliana. Methods Enzymol., 389: 338-350.

Chen, Y., Ji, F., Hong, X., Liang, J., and Zhang, J. (2006) The Regulator of GProtein Signaling Proteins Involved in Sugar and Abscic Acid Signaling in Arabidopsis Seed Germination. Plant Physiol., 14: 302-310.

Cheng, W., Endo, A., Zhou, L., Penny, J., Chen, H., Arroyo, A., Leno, P., Nambara, E., Patricia, L., Nambara, E., Asami, T., Seo, M., Koshiba, T., and Sheen, J. (2002) A Uniqe Short-Chain Dehydrogenase/Reductase in Arabidopsis Glucose Signaling and Abscisic Acid Biosynthesis and Functions. Plant Cell, 14: 2723-2743.

Cheung, P., Allis, C., and Sassone-Corsi, P. (2000) Signaling to chromatin through histone modifications. Plant Cell, 103:263-271. 
Chevalier, C., Bourgeois, E., Just, D., and Raymond, P. (1996) Metabolic regulation of asparagine synthetase gene expression in maize (Zea mays L.) root tips. Plant J., 9:1-11.

Chinnusamy, V., Gong, Z., and Zhu, J. (2008) Abscisic Acid-mediated Epigenetic Processes in Plant Development and Stress Responses. Plant Biol., 50:1187-1195.

Chiou, T., and Bush, D. (1998) Sucrose is a signal molecule in assimilate partitioning. Proc. Natl. Acad. Sci. USA, 95:4784-4788.

Chuang, P., Albertson, D., and Meyer, B. (1994) DPY-27: a chromosome condensation protein homolog that regulates $C$. elegans dosage compensation through association with the $\mathrm{X}$ chromosome. Plant Cell, 79:459-474.

Chuang, P., Lieb, J., and Meyer, B. (1996) Sex-specific assembly of a dosage compensation complex on the nematode $X$ chromosome. Science, 274:173639.

Colville, A.H. (2007) Sugar and HD2 Expression: New Insight into the HD2 plant-specific class of histone deacetylases (Master thesis). Carleton University.

Cortes, S., Gromova, M., Evrard, A., Roby, C., Heyraud, A., Rolin, D., Raymond, P., and Brouquisse, R. (2003) In plants, 3-0-methylglucose is phosphorylated by hexokinase but not perceived as a sugar. Plant Physiol., 131:824-837.

Criqui, M. C., Parmentier, Y., Derevier, A., Shen, W., Dong, A., and Genschik, P. (2000) Cell cycle-dependent proteolysis and ectopic overexpression of cyclin B1 in tobacco BY2 cells. Plant J., 24:763-773.

Dangl, M., Brosh, G., Haas, H., Loidl, P., and Lusser, A. (2001) Comparative analysis of HD2 type histone deacetylase in higher plants. Planta, 213:280-285.

Debeaujon, I., and Koornneef, M. (2000) Gibberellin requirement for Arabidopsis seed germination is determined both by testa characteristics and embryonic abscisic acid. Plant Physiol., 122:415-424.

Dekkers, B.J.W., Schuurmans, J.A.M.J., and Smeekens, S.C.M. (2004) Glucose delays seed germination in Arabidopsis thaliana. Planta, 218:579-588.

Dekkers, B.J.W., Schuurmans, J.A.M.J., and Smeekens, S.C.M. (2008) Interaction between sugar and abscisic acid signalling during early seedling development in Arabidopsis. Plant Mol. Biol., 67: 151-167. 
Dewitte, W., and Murray, J. (2003) The Plant cell cycle. Plant Biol., 54:235-264.

Den Boer, B., and Murray, J. (2000) Triggering the cell cycle in plants. Trends Cell Biol. 10:245-250.

Dure, L., (1993). A repeating 11-mer amino acid motif and plant desiccation. Plant J., 3:363-369.

Earley, K., Lawrence, R.J., Pontes, O., Reuther, R., Enciso, A.J., Silva, M, Neves, N., Gross, M., Viegas, W., and Pikaardl, C.S. (2006) Erasure of histone acetylation by Arabidopsis HDA6 mediates large-scale gene silencing in nucleolar dominance. Genes Dev., 20: 1238-1293.

Earnshaw, W., Halligan, B., Cooke, C., Heck, M., and Liu, L. (1985) Topoisomerase II is astructural component of mitotic chromosome scaffolds. J. Cell Biol., 100:1706-1715.

Ehness, R., and Entian, K. (1980). Genetic and biochemical evidence for hexokinase PII as a keyenzyme involved in carbon catabolite repression in yeast. Mol. Gen. Genet., 178:633-637.

Ehness, R., Ecker, M., Godt, D.E., and Roitsch, T. (1997) Glucose and stress independently regulate source and sink metabolism and defense mechanisms via signal transduction pathways involving protein phosphorylation. Plant Cell, 9: 1825-1841.

Entian, K., and Frhlich, K. (1984). Saccharomyces cemvisiae mutants provide evidence of hexokinase PII as a bifunctional enzyme with catalytic and regulatory domains for triggering carbon catabolite repression. J. Bacteriol., 158:29-35.

Farrar, J. (1991) Starch turnover: its role in source-sink relations and a comparison with the role of sucrose. In JL Bonnemain, SDelrot, WJ Lucas, J Dainty, eds, Recent Advances in PhloemTransport and Assimilate Compartmentation. Presses Academiques. Nantes, France, pp 213-223.

Felsenfeld, G., Boyes, J., Chung, J., Clark, D., and Studitsky, V. (1996) Chromatin structure and gene expression. Proc. Natl. Acad. Sci. USA, 93:93849388.

Finkelstein, R., Gampala, S., and Rock, C. (2002) Abscisic acid signaling in seeds. Plant Cell, 14:14-45.

Finkelstein, R., Reeves, W., Ariizumi, T., and Steber, C. (2008) Molecular aspects of seed dormancy. Annu. Rev. Plant Biol., 59: 387-415. 
Fong, P., Tian, L., and Chen, Z. (2006) Arabidopsis thaliana histone deacetylase 1 (AtHD1) is localized in euchromatic regions and demonstrates histone deacetylase activity in vitro. Cell Res., 16:479-488.

Ford, C.E., Skiba, N.P., Bae, H., Daaka, Y., Reuveny, E., and Shekter, L.R. (1998) Molecular basis for interactions of $G$ protein betagamma subunits with effectors. Science, 280: 1271-1274.

Fu, W., Wu, K., and Duan, J. (2007) Sequence and expression nalysis of histone deacetylases in rice. Biochem. Biophys. Res. Commun., 356: 843-850.

Gancedo, J. (1992) Carbon catabolite repression in yeast. Eur J Biochem., 206: 297-313.

Georgieva, E., Rodas, G., and Loidl, P. (1994) Maize embryo germination. Planta, 192: 125-129.

Gibson, S.I. (2000) Plant sugar-response pathways. Part of a complex regulatory web. Plant Physiol., 124: 1532-1539.

Gibson, S.I. (2004) Sugar and phytohormone response pathways: navigating a signalling network. J. Exp. Bot., 55: 253-264.

Gibson, S.I. (2005) Control of plant development and gene expression by sugar signalling. Curr. Opin. Plant Biol., 8: 93-102.

Gibson, S.I., Laby, R., and Kim, D. (2001) The sugar-insensitive1 (sis1) mutant of Arabidopsis is allelic to ctr1. Biochem Biophys Res Commun., 280:196-203.

Graham, I. (1996) Carbohydrate control of gene expression in higher plants. Res. Microbiol., 147:572-580.

Graham, I., Denby, K., and Leaver, C. (1994) Carbon catabolite repression regulates glyoxylate cycle gene expression in cucumber. Plant Cell, 6:761-772.

Goldberg, R., de Pavia, G., and Yadegari, R. (1994). Plant embryogenesis: Zygote to seed. Science, 266:605-614.

Gudermann, T., Schoneberg, T., and Schultz., G. (1997) Functional and structural complexity of signal transduction via G-protein-coupled receptors. Annu. Rev. Neurosci., 20: 399-427.

Gutierrez, C., Ramirez-Parra, E., Castellano, M., and del Pozo, J. (2002) G1 to $S$ transition: more than a cell cycle engine switch. Curr. Opin. Plant Biol., 5:480-486. 
Hattori, T., Fukumoto, H., Nakagawa, S., and Nakamura, K. (1991) Sucroseinduced expression of genes coding for the tuberous root storage protein, sporamin, of sweet potato in leaves and petioles. Plant Cell Physiol., 32:79-86.

Hattori, T., Nakagawa, S., and Nakamura, K. (1990) High-level expression of tuberous root storage protein genes of sweet potato in stems of plantlets grown in vitro on sucrose medium. Plant Mol. Biol., 14: 595-604

Hartig, K., and Beck, E. (2005) Endogenous Cytokinin Oscillations Control Cell Cycle Progression of Tobacco BY-2 Cells. Plant Biol.,7:33-40.

Heitz, E. (1928) Das Heterochromatin der Moose. I. Jahrb. Wiss. Bot., 69:762819.

Henderson, J.T., Li, H.C., Rider, S.D., Mordhorst, A.P., Romero-Severson, J., Cheng, J.C., Robey, J., Sung, R., Vries, S.C., and Ogas, J. (2004) PICKLE acts throughout the plant to repress expression of embryonic traits and may play a role in gibberellin dependent responses. Plant Physiol., 134: 995-1005.

Hills, M. (2004) Control of storage-product synthesis in seeds. Curr. Opin. Plant Biol., 7:302-308.

Hill, K., Wang, H., and Perry, S. (2008) A transcriptional repression motif in the MADS factor AGL15 is involved in recruitment of histone deacetylase complex components. Plant J., 53:172-185.

Hirano, H., Harashima, H., Shinmyo, A., and Sekine, M. (2008) Arabidopsis RETINOBLASTOMA-RELATED PROTEIN 1 is involved in $\mathrm{G} 1$ phase cell cycle arrest caused by sucrose starvation. Plant Mol. Biol., 66: 259-275.

Hirano, T. (1999) SMX-mediated chromosome mechanics: a conserved scheme from bacteria to vertebrates?. Genes Dev., 13: 11-19.

Ito, T., Bulger, M., Pazin, M., Kobayashi, R., and Kadonaga, J. (1997) ACF, an ISWI-containing and ATP-utilizing chromatin assembly and remodeling factor. Plant Cell, 90:145-155.

Inze, D. (2005) Green light for cell cycle. EMBO J., 24:657-662.

Jang, J., and Sheen, J. (1997) Sugar sensing in higher plants. Trends Plant Sci., 2:208-214.

Jang, J., LeoÂn, P., Zhou, L., and Sheen, J. (1997) Hexokinase as a sugar sensor in higher plants. Plant Cell, 9:5-19. 
Jang, J., and Sheen, J. (1994) Sugar sensing in higher plants. Plant Cell, 6:1665-1679.

Jefferson, R.A., Kavanagh, T.A., and Bevan, M.W. (1987) GUS fusion: $\beta$ glucuronidese as a sensitive and versatile gene fusion marker in higher plants. $E M B O$ J., 6: 3901-3907.

Johnston, C.A., Taylor, J.P., Gao, Y., Kimple, A.J., Grigston, J.C., Chen, J.G., Siderovski, D.P, Jones, A.M., and Willard, F.S. (2007) GTPase acceleration as the rate-limiting step in Arabidopsis $G$ protein coupled sugar signaling. Proc. Natl. Acad. Sci. USA, 104: 17317-17222.

Johnston, M. (1999) Feasting, fasting and fermenting-glucose sensing in yeast and other cells. Trends Genet., 15: 29-33.

Johnston, M., and Carlson, M. (1992) Regulation of carbon and phosphate utilization. In EW Jones, JR Pringle, JR Broach, eds, The Molecular and Cellular Biology of the Yeast Saccharomyces, Vol 2. Cold Spring Harbor Laboratory Press, Cold Spring Harbor, NY, pp. 193-281.

Kaszas, E., and Cande, W. (2000) Phosphorylation of histone H3 is correlated with changes in the maintenance of sister chromatid cohesion during meiosis in maize, rather than the condensation of the chromatin. J. Cell Sci., 113:3217-26.

Karrer, E., and Rodriguez, R. (1992) Metabolic regulation of rice $\alpha$-amylase and sucrose synthase genes in planta. Plant J., 2:517-523.

Keith, K., Kraml, M., Dengler, N., and McCourt, P. (1994) fusca3: A Heterochronic Mutation Affecting Late Embryo Development in Arabidopsis. Plant Cell, 6:589-600.

Kermode, A. (1990) Regulatory mechanisms involved in the transition from seed development to germination. Crit. Rev. Plant Sci., 9:155-196.

Kim, H.J., Hyun, Y., Park, J.Y., Park, M.J., Park, M.K., Kim, M.D., Lee, M.H., Moon, J., Lee, I., and Kim, J. (2004) A genetic link between cold responses and flowering time through FVE in Arabidopsis thaliana. Nat Genet., 36: 167171.

Koch, K. (1996) Carbohydrate-modulated gene expression in plants. Annu. Rev. Plant Physiol. Mol. Biol., 47:509-540.

Kornberg, R., and Klug, A. (1981) The nucleosome. Sci. Am., 244:52-64. 
Koornneef, M., Jorna, M., Brinkhorst-Van der Swan, D., and Karssen, C. (1982) The isolation of abscisic acid (ABA) deficient mutants by selection of induced revertants in non-germinating gibberellin sensitive lines of Arabidopsis thaliana (L.) heynh. Theor. Appl. Genet., 61:385-393.

Koorneef, M., and Karssen, C. (1994) Seed dormancy and germination in Arabidopsis. (ed. E.M. Meyerowitz and C.R. Somerville), pp. 313-334. Cold Spring Harbor Laboratory Press, Cold Spring Harbor, NY.

Koornneef, M., Reuling, G., and Karssen, C. (1984) The isolation and characterisation of abscisic acid insensitive mutants of Arabidopsis thaliana. Plant Physiol., 61:377-383.

Krapp, A., Hofmann, B., Schafer, C., and Stitt, M. (1993) Regulation of the expression of rbcS and other photosynthetic genes by carbohydrates: a mechanism for the "sink regulation" of photosynthesis? Plant J., 3:817-828.

Kushiro, T., Okamoto, M., Nakabayashi, K., Yamagishi, K., Kitamura, S., Asami, T., Hirai, N., Koshiba, T., Kamiya, Y., and Nambara, E. (2004) The Arabidopsis cytochrome P450 CYP707A encodes ABA 8 2- hydroxylases: key enzymes in ABA catabolism, EMBO J., 23: 1647-1656.

Laby, R., Kincaid, M., Kim, D., and Gibson, S. (2000) The Arabidopsis sugarinsensitive mutants sis 4 and sis 5 are defective in abscisic acid synthesis and response. Plant J., 23:587-596.

Lagacé, M., Chantha, S.C., Major, G., and Matton, D.P. (2003) Fertilization induces strong accumulation of a histone deacetylase (HD2) and of other chromatin-remodeling proteins in restricted areas of the ovules. Plant Mol. Biol., 53: $759-769$.

Lalonde, S., Boles, E., Hellmann, H., Barker, L., Patrick, J.W., Fommer, W.B., and Ward, J.M. (1999) The dual function of sugar carriers: transport and sugar sensing. Plant Cell, 11: 707-726.

LeÂon, P., and Sheen J (2003) Sugar and hormone connections. Trends Plant Sci., 8:110-116.

Lee, B., Henderson, D.A., and Zhu, J.K. (2005) The Arabidopsis cold responsive transcriptome and its regulation by ICE1. Plant Cell, 11: 3155-3175.

Li, Y., Lee, K., Walsh, S., Smith, C., Hadingham, S., Sorefan, K., Cawley, G., and Bevan, M. (2006) Establishing glucose-and ABA-regulated transcription netweorks in Arabidopsis by microarray analysis amd promoter classification using a Relevance Vector Machine. Genome Res., 16:414-427. 
Li, Y., Sternweis, P.M., Charnecki, S., Smith, T.F., Gilman, A.G., and Neer, E.J. (1998) Sites for $G$ alpha binding on the $G$ protein beta subunit overlap with sites for regulation of phospholipase $C$ beta and adenylyl cyclase. Biol. Chem., 273: 16265-16272.

Liu, X., Yue, Y., Li, B., Nie, Y., Li, W., Wu, W.H and Ma, L. (2007) A G proteincoupled receptor is a plasma membrane recptor is a plasma membrane receptor for the plant hormone abscisic acid. Science, 315: 1712-1716.

Leon-Kloosterziel, K.M., Gil, M.A., Ruijs, G.J., Jacobsen, S.E., Olszewski, N.E., Schwartz, S.H., Zeevaart, J.A., Koornneef, M. (1996) Isolation and characterization of abscisic acid-deficient Arabidopsis mutants at two new loci. Plant J., 10: 655-661.

Loidl, P. (2004) A Plant dialect of the histone language. Trends Plant Sci., 9:8490.

Loidl, P. (1988) Towards an understanding of the biological function of histone acetylation. FEBS Lett., 227: 91-95.

Lotan, T., Ohto, M., Yee, K.M., West, M.A., Lo, R., Kwong, R.W., Tamagishi, K., Fisher, R.L., Golberg, R.B., and Harada, J.J. (1998) Arabidopsis LEAFY COTYLEDON 1 is sufficient to induce embryo development in vegetative cells. Cell, 93: 1195-1205.

Lupo, R., Breiling, A., Bianchi, M.E., and Orlando, V. (2001) Drosophila chromosome condensation proteins Topisomerase II and Barren co-localize with Polycomb and maintain Fab-7 PRE silencing. Mol. Cell, 7:127-136.

Lusser, A., Kolle, D., and Loidl, P. (2001) Histone acetylation: lessons from the plant kingdom. Trends Plant Sci., 6:59-65.

Lusser, A., Brosh, G., Loidl, A., Haas, H., and Loidl, P. (1997) Identification of maize histone deacetylase HD2 as an acidic nucleolar phosphoprotein. Science, 277:88-91.

Ma, H., and Botstein, D. (1986) Effects of null mutations in the hexokinase genes of Saccharomyces cerevisiae on catabolite repression. Mol. Cell. Biol., 6:4046-4052.

Magasanik, B. (1961) Catabolite repression. Cold Spring Harbor Symp Quant Biol., 26:193-197.

Manevski, A., Bertoni, G., Bardet, C., Tremousaygue, D., and Lescure, B. (2000) In synergy with various cis-acting elements, plant insterstitial telomere 
motifs regulate gene expression in Arabidopsis root meristems. FEBS Lett., 483: 43-46.

Martin, T., Hellmann, H., Schmidt, R., Willmitzer, L., and Frommer, W. (1997) Identification of mutants in metabolically regulated gene expression. Plant J., 11:53-62.

McLaughlin, J., and Smith, S. (1994) Metabolic regulation of glyoxylate-cycle enzyme synthesis in detached cucumber cotyledons and protoplasts. Planta, 195:22-28.

McCarty, D. (1995). Genetic control and integration of maturation and germination pathways in seed development. Annu. Rev. Plant Physiol. Plant Mol. Biol., 46:71-93.

Meijer, M., and Murray, J. (2000) The role and regulation of D-type cyclins in the plants cell cycle. Plant Mol. Biol., 43:621-633.

Meinke, D. (1992) A homeotic mutant of Arabidopsis thaliana with leafy cotyledons. Science, 258:1647-1650.

Meinke, D., Franzmann, L., Nickle, T., and Yeung, E. (1994) Leafy Cotyledon mutants of Arabidopsis Plant. Plant Cell, 6:1049-1064.

Mita, S., Murano, N., Akaike, M., and Nakamura, K. (1997) Mutants of Arabidopsis thaliana with pleiotropic effects on the expression of the gene for $\beta$ amylases and on the accumulation of anthocyanin that are inducible by sugars. Plant J., 11: 841-851.

Millar, A.A., Jacobsen, J.V., Ross, J.J., Helliwell, C.A., Poole, A.T., Scofield, G., Reid, H.B., and Gubler, F. (2006) Seed dormancy and ABA metabolism in Arabidopsis and barley: the role of ABA 8 2-hydroxylase. Plant J., 45: 942-954.

Mironov, V., de Veylder, L., van Montagu, M., and Inze, D. (1999) Cyclindependent kinases and cell division in plants-The nexus. Plant Cell, 11:509-521.

Moore, B. and Sheen, J. (1999) Plant sugar and signaling: a complex reality. Trends Plant Sci., 4: 248-249.

Moore, B., Zhou, L., Rolland, F., Hall, Q., Cheng, W., Liu, Y., Hwang, I., Jones, T., and Sheen, J. (2003) Role of the Arabidopsis glucose sensor HXK1 in nutrient, light, and hormonal signalling. Science, 300:332-336.

MuĖller-RoÈber, B., Sonnewald, U., and Willmitzer, L. (1992) Inhibition of the ADP-glucose pyrophosphorylase in transgenic potatoes leads to sugar-storing 
tubers and in uences tuber formation and expression of tuber storage protein genes. EMBO J.,11:1229-1238.

Nakamura, K., Ohto, M., Yoshida, N., and Nakamura, K. (1991) Sucroseinduced accumulation of a-amylase occurs concomitant with the accumulation of starch and sporamin in leaf-petiole cuttings of sweet potato. Plant Physiol., 96: 902-909.

Nicolai, M., Roncato, M., Canoy, A., Rouquie, D., Sarda, X., Freyssinet, G., and Robaglia, C. (2006) Large-scale analysis of mRNA translation states during aucrose starvation in Arabidopsis cells identifies cell proliferation and chromatin structure as targets of translational control. Plant Physiol., 141:663673.

Ogas, J., Cheng, J., Sung, Z., and Somerville, C. (1997) Cellular differentiation regulated by gibberellin in the Arabidopsis thaliana pickle mutant. Science, 277:91-94.

Ogas, J., Kaufmann, S., Henderson, J., and Somerville, C. (1999) PICKLE is a CHD3 chromatin-remodeling factor that regulates the transition from embryonic to vegetative development in Arabidopsis. Proc Natl Acad Sci USA, 96:13839-13844.

Okamoto, M., Kuwahara, A., Seo, M., Kushiro, T., Asami, T., Hirai, N., Kamiya, Y., Koshiba, T., and Nambara, E. (2006) CYP707A1 and CYP707A2, which encode abscisic acid 8 2-hydroxylases, are indispensable for proper control of seed dormancy and germination in Arabidopsis. Plant Physiol., 141: 97-107.

O"zcan, S., Dover, J., Rosenwald, A., Wolfl, S., and Johnston, M. (1996) Two glucose transporters in Saccharomyces cerevisiae are glucose sensors that generate a signal for induction of geneexpression. Proc. Natl. Acad. Sci. USA, 93:12428-12432.

Pandey, R., Muller, A., Napoli, C., Selinger, D., Pikaard, C., Richards, E., Mount, D., and Jorgensen R. (2002) Analysis of histone acetyltransferase and histone deacetylase families of Arabidopsis thaliana suggests functional diversification of chromatin modification among multicellular eukaryotes. Nucleic Acids Res.,30:5036-5055.

Parcy, F., Valon, C., Kohara, A., Misera, S., and Giraudat, J. (1997) The ABSCISIC ACID-INSENSITIVE3, FUSCA3, and LEAFY COTYLEDON1 loci act in concert to control multiple aspects of Arabidopsis seed development. Plant Cell, 9:1265-1277. 
Patonnier, M.P., Peltier, J.P., and Marigo, G. (1999) Drought-induced increase in xylem malate and mannitol concentration and closure of Fraxinus exce/sior $\mathrm{L}$. stomata. J. Exp. Bot., 50: 1223-1229.

Pego, J., Weisbeek, P., and Smeekens, S. (1999) Mannose inhibits Arabidopsis germination via a hexokinase-mediated step. Plant Physiol., 119:1017-1023.

Pego, J., Kortstee, A., Huijser, G., and Smeekens, S. (2000) Photosynthesis, sugars and the regulation of gene expression. J. Exp. Bot., 51: 407-416.

Peterson, C., and Laniel, M. (2004) Histones and histone modifications. Curr. Opin. Plant Biol., 14:R546-R551.

Perruc, E., Kinoshita, N., and Lopez-Molina, L. (2007) The role of chromatinremodeling factor PKL in balancing osmotic stress responses during Arabidopsis seed germination. Plant J., 52: 927-936.

Prata, R., Williamson, J., Conkling, M., and Pharr, D. (1997) Sugar repression of mannitol dehydrogenase activity in celery cells. Plant Physiol., 114: 307-314.

Price, J., Laxmi, A., St Martin, S.K., and Jang, J.C. (2004) Global transcription profiling reveals multiple sugar signal transduction mechanisms in Arabidopsis. Plant Cell,16: 2128-2150.

Price, J., Li, T., Kang, S., Na, J., and Yang, J. (2003) Mechanisms of glucose signaling during germination of Arabidopsis. Plant Physiol., 132:1424-1438.

Probst, A.V., Fagard, M., Proux, F., Mourrain, P., Boutet, S., Earley, K., Lawrence, R.J., Pikaard, C.S., Murfett, J., Furner, I., Vaucheret, H., and Scheid, O.M. (2004) Arabidopsis histone deacetylase HDA6 is required for maintenance of transcription gene silencing and determines nuclear organization of rDNA repeats. Plant Cell,16: 1021-1034.

Quesada, V., Ponce, M., and Micol, J. (2000) Genetic analysis of salt-tolerant mutants in Arabidopsis thaliana. Genetics., 154:421-436.

Ray, T.B. (1984) Site of Action of Chlorsulfuron: Inhibition of Valine and Isoleucine Biosynthesis in Plants. Plant Physiol., 75: 827-831.

Reichheld, J., Chaubet, N., Shen, W., Renaudin, J., and Gigot, C. (1996) Multiple A type cyclins express sequentially during the cell cycle in Nicotiana tabacum BY2 cells. Proc. Natl. Acad. Sci., 93:13819-13824.

Reyes, J.C. (2006) Chromatin modifiers that control plant development. Plant Biol., 9:21-27. 
Reyes, J.C., Hennig, L., and Gruissem, W. (2002) Chromatin-Remodeling and Memory Factors: New Regulators of Plant Development. Plant Physiol., 130: 1090-1101.

Rider, S., Henderson, J., Jerome, R., Edenberg, H., Romero-Severson, J., and Ogas, J. (2003) Coordinate repression of regulators of embryonic identity by PICKLE during germination in Arabidopsis. Plant J., 35:33-43.

Riou-Khamlichi, C., Menges, M., Healy, J.M.S., and Murray, J.A.H. (2000) Sugar control of the plant cell cycle: differential regulation of Arabidopsis D-type cyclin gene expression. Mol. Cell. Biol., 20: 4513-4521.

Rock, C. and Quatrano, R. (1995) The role of hormones during seed development. In P. Davies, ed. Plant Hormones: Physiology, Biochemistry and Molecular Biology. Kluwer Academic Publishers, Dordrecht, The Netherlands. pp. 671-697.

Rolland, F., Moore, B., and Sheen, J. (2002) Sugar sensing and signaling in plants. Plant Cell, 14:185-S205.

Rolland, F., Gonzalez, E., and Sheen, J. (2006) Sugar Sensing and Signaling in Plants: Conserved and Novel Mechanisms. Plant Biol., 57:675-709.

Rolland, F., Winderickx, J., and Thevelein, J.M (2002) Glucose-sensing and signaling mechanisms in yeast. FEMS Yeast Res., 2:183-201.

Rolland, F., Winderickx, J., and Thevelein, J.M (2001) Glucose-sensing mechanisms in eukaryotic cells. Trends Biochem. Sci., 26: 310-317.

Roitsch, T., Bittner, M., and Godt, D. (1995) Induction of Chenopodium rubrum by $D$-glucose and a glucose analog and tissue-specific expression suggest a role in sink-source regulation. Plant Physiol., 108:285-294.

Roitsch, T. (1999). Source-sink regulation by sugar and stress. Curr. Opin. Plant Biol., 2:198-206.

Ronne, H. (1995) Glucose repression in fungi. Trends Genet., 11:12-17.

Rook, F., and Bevan, M. (2003) Genetic approaches to understanding sugarresponse pathways. J. Exp. Bot., 54: 495-501.

Rook, F., Gerrits, N., Kortstee, A., van Kampen, M., Borrias, M., Weisbeek, P., and Smeekens, S. (1998) Sucrose-speci®c signaling represses translation of the Arabidopsis ATB2 bZIP transcription factor gene. Plant J.,15: 253-263. 
Rook, F., Hadingham, S.A., Li, Y., and Bevan, M.W. (2006) Sugar and ABA response pathways and the control of gene expression. Plant Cell Environ., 29: 426-434.

Rose, M., Albig, W., and Entian, K. (1991). Glucose repression in Saccharomyces cerevisiae is directly associated with hexose phosphorylation by hexokinase PI and PII. Eur. J. Biochem., 199:511-518.

Rossi, V., Locatelli, S., Lanzanova, C., Boniotti, M., Varotto, S., Pipal, A., Goralik, M., Lusser, A., Gatz, C., Gutierrez, C., and Motto, M. (2003) A maize histone deacetylase and retinoblastoma-related protein physically interact and cooperate in repressing gene transcription. Plant Mol. Biol., 51:401-413.

Rossi, V., and Varotto, S. (2002) Insights into the G1/S transition in plants. Planta, 215:345-356.

Rundlett, S.E., Carmen, A.A., Kobayashi, R., Bavykin, S., Turner, B.M., and Grunstein, M. (1996) HDA1 and RPD3 are members of distinct yeast histone deacetylase complexes that regulate silencing and transcription. Proc. Natl. Acad. Sci. USA, 93: 14503-14508.

Saier, M. (1989) Protein phosphorylation and allosteric control of inducer exclusion and catabolite repression by the bacterial phosphoenolpyruvate: sugar phosphotransferase system. Microbiol Rev., 53: 109-120.

Saito, S., Hirai, N., Matsumoto, C., Ohigashi, H., Ohta, D., Sakata, K., and Mizutani, M. (2004) Arabidopsis CYP707As encode (+)-abscisic acid 82 2hydroxylase, a key enzyme in the oxidative catabolism of abscisic acid. Plant Physiol., 134: 1439-1449.

Scheible, W.R., Eshed, R., Richmond, T., Delmer, D., and Somerville, C. (2001) Modifications of cellulose synthase confer resistance to isoxaben and thiazolidinone herbicides in Arabidopsis Ixr1 mutants. Proc. Natl. Acad. Sci. USA 93: 14503-14508.

Schroeder, J.I., Kwak, J.M., and Allen, G.J. (2001) Guard cell abscisic acid signaling and engineering drought hardiness in plants. Nature, 410: 327-330.

Schwebel-Dugué, N., El Mtili', N., Krivitzky, M., Jean-Jacques, l., Williams, J., Thomas, M., Kreis, M., and Lecharny, A. (1994) Arabidopsis Gene and cDNA Encoding Cell-Wall Innvertase. Plant Physiol., 104:809-810.

Shaked, H., Avivi-Ragolsky, N., and Levy, A. (2006) Involvement of the Arabidopsis SWI2/SNF2 chromatin remodeling gene family in DNA damage response and recombination. Genetics., 173: 985-994. 
Shaner, D.L., Anderson, P.C., and Stidham, M.A. (1984) Imidazolinones: Potent Inhibitors of Acetohydroxyacid Synthase. Plant Physiol., 76: 545-546.

Shen, Y.Y., Wang, X.F., Wu., F.Q., Du., Cao, Z., Shang, Y., Wang, X.L., Peng, C.C., Yu, X.C., Zhu, S.Y, Fan, R.C., Xu, Y.H., and Zhang, D.P. (2006) The $\mathrm{Mg}$-chelatase $\mathrm{H}$ subunit is an abscisic acid receptor. Nature, 443: 823-826.

Sheen, J. (1994) Feedback control of gene expression. Photosynth. Res., 39: 427-438.

Sheen, J., Zhou, L., and Jang, J. (1999) Sugars as signaling molecules. Curr. Opin. Plant Biol., 2:410-418

Sirdha, S., and Wu, K. (2006) Identification of AtHD2C as a novel regulator of abscisic acid responses in Arabidopsis. Plant J., 46:124-133.

Smeekens, S. (2000) Sugar-induced signal transduction in plants. Annu.Rev. Plant Biol., 51:49-81.

Smeekens, S. (1998) Sugar regulation of gene expression in plants. Curr. Opin. Plant Biol., 1:230-234.

Smeekens, S., and Rook, F. (1997) Sugar sensing and sugar-mediated signal transduction in plants. Plant Physiol., 115:7-13.

Smith, A., Zeeman, S., and Smith, S. (2005) Starch degradation. Annu. Rev. Plant Biol., 56:73-98.

Song, C.P., Agarwal, M., Ohta, M., Guo, Y., Halfter, U., Wang, P and Zhu, J.K. (2005) Role of an Arabidopsis AP2/EREBP-type transcriptional repressor in abscisic acid and drought stress responses. Plant Cell, 17: 2384-2396.

Sorrell, D., Combetter, B., Chaubet-Gigot, N., Gigot, C., and Murray, J. (1999) Distinct cyclin $D$ genes show mitotic accumulation or constant levels of transcripts in tobacco Bright Yellow-2 cells. Plant Physiol., 119:343-351.

Stals, H., and Inze, D. (2001) When plant cells decide to divide. Trends Plant Sci., 6:359-364.

Stockinger, E.J., Mao, Y., Regier, M.K., Triezenberg, S.J., and Thomashow, M.F. (2001) Transcriptional adaptor and histone acetylransferase proteins in Arabidopsis and their interactions with CBF1, a transcriptional activator involved in cold-regulated gene expression. Nucleic Acids Res., 29: 1524-1533.

Stone, S.L., Kwong, L.W., Yee, K.M., Pelletier, J., Lepiniec, L., Fischer, R.L., Goldberg, R.B., Harada, J.J. (2001) LEAFY COTYLEDON2 encodes a B3 
domain transcription factor that induces embryo development. Procd. Natl. Acad. Sci. USA, 98: 11806-11811.

Strahl, B.D., and Allis, C.D. (2000) The language of co-valent histone modifications. Nature, 403: 41-45.

Takahashi, F., Sato-Nara, K., Kobayashi, K., Suzuki, M., and Suzuki, H. (2003) Sugar-induced adventitious roots in Arabidopsis seedlings. J Plant Res., 116: 83-91.

Tanaka, M., Kikuchi, A., and Kamada, H. (2008) The Arabidopsis histone deacetylases HDA6 and HDA19 contribute to the repression of the embryonic properties after germination. Plant Physiol., 146:149-161.

Tatematsu, K., Ward, S., Leyser, O., Kamiya, Y., and Nambara, E (2005) Identification of cis-elements that regulate gene expression during initiation of axillary bud outgrowth in Arabidopsis. Plant Physiol., 138:757-766.

Tariq, M., and Paszkowski, J. (2004) DNA and histone methylation in plants. Trends Plant Sci., 20: 6.

Thevelein, J. (1994) Signal transduction in yeast. Yeast, 10:1753-1790.

Tian, L., and Chen, Z. (2001) Blocking histone deacetylation in Arabidopsis induces pleiotropic effects on plant gene regulation and development. Proc. Natl. Acad. Sci. USA., 98:200-205.

Tian, L., Fong, M., Wang, J., Wei, N., Jing, H., and Doerge, R. (2005) Reversible histone aceylation and deacetylation mediate genome-wide, promoter-dependent and locus-specific changes in gene expression during plant development. Genetics., 169:337-345.

Tong, J.K., Hassig, C.A., Schnitzler, G.R., Kingston, E.R., and Schreiber, S.L. (1998) Chromatin deacetylation by an ATP-dependent nucleosome remodeling complex. Nature, 395: 917-921.

Tsukagoshi, H., Morikami, A., and Nakamura, K. (2007) Two B3 domain transcriptional repressors prevent sugar-inducible expression of seed maturation genes in Arabidopsis seedlings. Proc Natl Acad Sci USA, 104:25432547.

Tsukiyama, T., and Wu, C. (1995) Purification and properties of an ATPdependent nucleosome remodeling factor. Cell, 83:1011-1020.

Trumbly RJ (1992) Glucose repression in yeast Saccharomyces cerevisiae. Mol Microbiol., 6:15-21. 
Tu, J., and Carlson, M. (1995) REG1 binds to protein phosphatase type 1 and regulates glucose repression in Saccharomyces cerevisiae. EMBO J., 14:59395946.

Ullmann, A. (1985) Catabolite repression. Biochimie., 67:29-34.

Ulker, B., Peiter, E., Dixon, D.P., Moffat, C., Capper, R., Bouche, N., Edwards, R., Sanders, D., Knight, H., and Knight, M.R. (2008) Getting the most out of publicly available T-DNA insertion lines. Plant J., 56:665-677.

Varga-Weisz, P., Wilm, M., Bonte, E., Dumas, K., Mann, M., and Becker, P. (1997) Chromatin-remodeling factor CHRAC contains he ATPases ISWI and topoisomerase II. Nature., 388:598-602.

Verdone, L., Camilioni, G., Dimauro, E., and Casertas, M. (1996) Chromatin Remodeling during Saccharomyces cerevisiae ADH2 Gene Activation. Mol. Cell. Biol., 16:1978-1988.

Vignali, M., Hassan, A., Neely, K., and Workman, J. (2000) ATP-Dependent Chromatin -Remodeling Complexes. Mol. Cell. Biol., 20:1899-1910.

Vlachonasios, K.E., Thomashow, M.F., and Triezenberg, S.J. (2003) Disruption mutations of ADA2b and GCN5 transcriptional adaptor genes dramatically affect Arabidopsis growth, development, and gene expression. Plant Cell, 15: 626-638.

Wade, P. , Jones, P. , Vermark, D. , and Wolffe, A. (1998) A multiple subunit Mi-2 histone deacetylase from Xenopus laevis cofractionates with an associated Snf2 superfamily ATPase. Curr. Opin. Plant Biol., 8:843-846.

Wall, M.A., Posner, B.A., and Sprang, S.R. (1998) Structural basis of activity and subunit recognition in $\mathrm{G}$ protein heterotrimers. Structure, 6: 1169-1183.

Wagner, D. (2003) Chromatin regulation of plant development. Curr. Opin. Plant Biol., 6:20-28.

Wagner, D., and Meyerowitz, E.M. (2002). SPLAYED, a novel SWI/SNF ATPase homolog, controls reproductive development in Arabidopsis. Curr. Opin. Plant Biol., 12: 85-94.

Wang, H., Huang, Z., Xia, L., Feng, Q., Erdjument-Bromage, H., Strahl, B., Briggs, S., Allis, C., Wong, J., Tempst, P., and Zhang, Y. (2001) Methylation of histone $\mathrm{H} 4$ at arginine 3 facilitating transcriptional activation by nuclear hormone receptor. Science, 293:853-57. 
Wassenger, M. (2000) RNA-directed DNA methylation. Plant Mol. Biol.,43: 203220.

Weber, H., Borisjuk, L., and Wobus, U. (2005). Molecular physiology of legume seed development. Annu. Rev. Plant Biol., 56:253-79.

West, M., Yee, K., Danao, J., Zimmerman, J., Fischer, R., Goldberg, R., and Harada, J. (1994) LEAFYCOTYLEDON1 is an essential regulator of late embryogenesis and cotyledon identity in Arabidopsis. Plant Cell, 6:1731-1745.

Wingler, A., Purdt, S., MacLean, J.A., and Pourtau, N. (2006) The role of sugars in integrating environmental signals during the regulation of leaf senescence. J. Exp. Bot., 57: 391-399.

Whitlock, J., and Simpson, R. (1977) Localization of the sites along nucleosome DNA which interact with $\mathrm{NH} 2$-terminal histone regions. J. Biol. Chem., 252:6516-6520.

Wobus, U., and Weber, H. (1999a) Sugars as signal molecules in plant seed Development. Biol. Chem., 380:937-944.

Wobus, U., and Weber, H. (1999b) Seed maturation: genetic programmes and control signals. Curr. Opin. Plant Biol., 2:33-38.

Wolffe, A. (1998) Chromatin: Structure and Function. San Diego, CA, Academic Press.

Wu, K., Malik, K., Tian, L., Brown, D., and Miki, B. (2000a) Functional analysis of a RPD3 histone deacetylase homologue in Arabidopsis thaliana. Plant Mol. Biol., 44:167-176.

Wu, K., Tian, L., Malik, K., Brown, D., and Miki, B. (2000b) Functional analysis of HD2 histone deacetylase homologues in Arabidopsis thaliana. Plant J., 22:19-27.

Wu, K., Tian, L., Zhou, C., Brown, D., and Miki., B. (2003) Repression of gene expression by Arabidopsis HD2 histone deacetylases. Plant J., 34:241-247.

Xiao, W., Sheen, J., and Jang, J. (2000) The role of hexokinase in plant sugar signal transduction and growth and development. Plant Mol. Biol., 44:451-461.

Xiong, L., Schumarker, K., and Zhu, J.K. (2002) Cell signaling during cold, drought and salt stress. Plant Cell, 14: 165-183. 
Xu, C., Liu, C., Wang, Y., Li, L., Chen, W., Xu, Z., and Bai, S. (2005) Histone acetylation affects expression of cellular patterning genes in the Arabidopsis root epidermis. Proc. Natl. Acad. Sci. USA., 102:14469-14474.

Xue, Y., Wong, J., Moreno, G., Young, M., Cote, J., and Wang, W. (1998) NURD, a novel complex with both ATP-dependent chromatin-remodeling and histone deacetylase activities. Mol. Cell., 2:851-861.

Yamagishi, K., Tatematsu, K., Yano, R., Preston, J., Kitamura, S., Takahashi, H., McCourt, P., Kamiya, Y., and Nambara, E. (2009) CHOTTO1, a double AP2 domain protein of Arabidopsis thaliana, regulates germination and seedling growth under excess supply of glucose and nitrate. Plant Cell Physiol., 50:330-340.

Yuan, K., and Wysocka-Diller, J. (2006) Phytohormone signaling pathways interact with sugars during seed germination and seedling development. J. Exp. Bot., 57: 3359-3367.

Yoshida, S. (2003) Molecular regulation of leaf senescence. Curr. Opin. Plant Biol., 6: 79-84.

Yoshida, M., Kijima, M., Akita, M., and Beppu, T. (1990) Potent and specific inhibition of mammalian histone deacetylase both in vivo and in vitro by trichostain A. J. Biol. Chem., 265:17174-17179.

Yu, Y., Steinmetz, A., Brown, S., and Shen, W. (2003) The tobacco A-type cyclin, Nicta; cycA3; 2 , at the nexus of cell division and differentiation. Plant Cell, 15:2763-2777.

Zhang, Y., LeRoy, G., Seelig, H., Lane, W., and Reinberg, D. (1998) The dermatomyositis specific autoantigen $\mathrm{Mi2}$ is a component of a complex containing histone deacetylase and nucleosome remodeling activites. Cell, 95:279-289.

Zhou, L., Jang, J., Jones, T., and Sheen, J. (1998) Glucose and etheylene signal transduction crosstalk revealed by an Arabidopsis glucose-insensitive mutant. Plant Biol., 95:10294-10299.

Zhou, C., Labbe, H., Sirdha, S., Wang, L., Tian, L., Latoszek-Green, M., Yang, Z., Brown, D., Miki, B., and Wu, K (2004) Expression and function of HD2type histone deacetylases in Arabidopsis development. Plant J., 38:715-724.

Zhu, G., Ye, N., and Zhang, J. (2009) Glucose-Induced Delay of Seed Germination in Rice is Mediated by the Suppression of ABA Catabolism Rather Than an Enhancement of ABA Biosynthesis. Plant Cell Physiol., 50: 644-651. 